\title{
Limits on the Usefulness of Random Oracles
}

\author{
Iftach Haitner ${ }^{* \dagger} \quad$ Eran Omri ${ }^{\ddagger}{ }^{\dagger} \quad$ Hila Zarosim ${ }^{\uparrow} \| \S$
}

January 14,2013

\begin{abstract}
In the random oracle model, parties are given oracle access to a random function (i.e., a uniformly chosen function from the set of all functions), and are assumed to have unbounded computational power (though they can only make a bounded number of oracle queries). This model provides powerful properties that allow proving the security of many protocols, even such that cannot be proved secure in the standard model (under any hardness assumption). The random oracle model is also used for showing that a given cryptographic primitive cannot be used in a black-box way to construct another primitive; in their seminal work, Impagliazzo and Rudich [STOC '89] showed that no key-agreement protocol exists in the random oracle model, yielding that key-agreement cannot be black-box reduced to one-way functions. Their work has a long line of followup works (Simon [EC '98], Gertner et al. [STOC '00] and Gennaro et al. [SICOMP '05], to name a few), showing that given oracle access to a certain type of function family (e.g., the family that "implements" public-key encryption) is not sufficient for building a given cryptographic primitive (e.g., oblivious transfer). Yet, the following question remained open:

What is the exact power of the random oracle model?

We make progress towards answering the above question, showing that, essentially, any no private input, semi-honest two-party functionality that can be securely implemented in the random oracle model, can be securely implemented information theoretically (where parties are assumed to be all powerful, and no oracle is given). We further generalize the above result to function families that provide some natural combinatorial property.

Our result immediately yields essentially that the only no-input functionalities that can be securely realized in the random oracle model (in the sense of secure function evaluation), are the trivial ones (ones that can be securely realized information theoretically). In addition, we use the recent information theoretic impossibility result of McGregor et al. [FOCS '10], to show the existence of functionalities (e.g., inner product) that cannot be computed both accurately and in a differentially private manner in the random oracle model; yielding that protocols for computing these functionalities cannot be black-box reduced to the existence of one-way functions.
\end{abstract}

Keywords: random oracles; black-box separations; one-way functions; differential privacy; key agreement

\footnotetext{
${ }^{*}$ School of Computer Science, Tel Aviv University. E-mail: iftachh@cs.tau.ac.il.

${ }^{\dagger}$ Supported by the Israeli Centers of Research Excellence (I-CORE) program (Center No. 4/11).

${ }^{\ddagger}$ Department of Mathematics and Computer Science, Ariel University Center. E-mail: omrier@gmail.com. Research was done while Eran Omri was at Bar Ilan University.

${ }^{\S}$ Supported by the Israel Science Foundation (ISF) (Grant No. 189/11)

`Department of Computer Science, Bar Ilan University. E-mail: zarosih@cs.biu.ac.il.

${ }^{\|}$Hila Zarosim is grateful to the Azrieli Foundation for the award of an Azrieli Fellowship.
} 


\section{Contents}

1 Introduction $\quad 2$

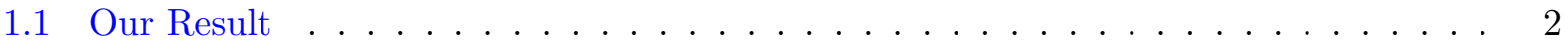

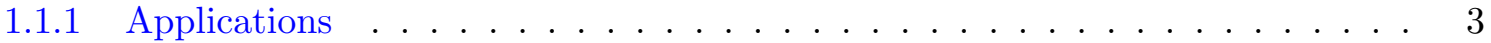

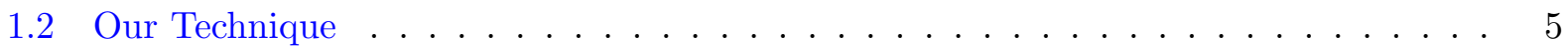

1.3 Related Work . . . . . . . . . . . . . . . . . . . . . 6

1.3.1 Additional Black-box Separations . . . . . . . . . . . . . . . . . 6

1.3.2 Differential Privacy . . . . . . . . . . . . . . . . . . . 6

1.4 Open Problems . . . . . . . . . . . . . . . . . . . . . . . 7

2 Preliminaries $\quad 7$

2.1 Notations . . . . . . . . . . . . . . . . . . . . . . . 7

2.2 Interactive Protocols . . . . . . . . . . . . . . . . . . . . 7

2.2 .1 Oracle-Aided Protocols . . . . . . . . . . . . . . . . . . 8

3 Mapping Oracle-Aided Protocols to No-Oracle Protocols 9

3.1 Dependent Views . . . . . . . . . . . . . . . . . . . . . . . . 9 9

3.2 Intersecting Views . . . . . . . . . . . . . . . . . . . . . . . 11

3.3 Oracle-Aided to No-Oracle Protocol Mapping . . . . . . . . . . . . . . . . 11

3.3 .1 Proving Theorem $12 \ldots \ldots \ldots \ldots \ldots \ldots$

4 Applications $\quad 15$

4.1 Key Agreement Protocols . . . . . . . . . . . . . . . . . . . . . . . . 15

4.1.1 Standard Definitions and Known Facts . . . . . . . . . . . . . . . . 15

4.1.2 Limits on Oracle-Aided Key-Agreement Protocols . . . . . . . . . . . . . . 16

4.2 Differentially Private Two-Party Computation . . . . . . . . . . . . . . . 17

4.2.1 Standard Definitions and Known Facts . . . . . . . . . . . . . . . . . 18

4.2.2 Limits on Differentially Private Oracle-Aided Protocols for Computing Inner

Product . . . . . . . . . . . . . . . . . . . 21

4.2.3 Limits on Sampled-Input Protocols . . . . . . . . . . . . . . . . . . . . 22

4.2.4 Limits on Uniform-Input Executions . . . . . . . . . . . . . . . . . . 28

4.2.5 Limits on Arbitrary Protocols - Proving Theorem 35 . . . . . . . . . . . . 29

4.3 Secure Function Evaluation . . . . . . . . . . . . . . . . . 30

4.3 .1 Standard Definitions . . . . . . . . . . . . . . . . 30

4.3.2 Limits on Oracle-Aided Secure Function Evaluation . . . . . . . . . . . . . 30

4.4 Applications to Random Functions, and Black-Box Reductions to One-way

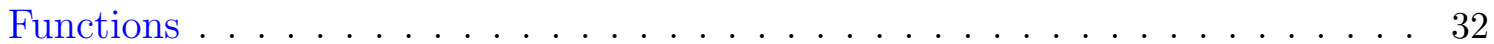

4.4.1 Standard Definitions and Known Facts . . . . . . . . . . . . . . . . . . . . . . . . . . . . . . . .

4.4.2 The All-Function Family is Simple . . . . . . . . . . . . . . . . . 33

4.4.3 Key Agreement Protocols . . . . . . . . . . . . . . . . . . . . . . 34

4.4.4 Differentially Private Two-Party Computation . . . . . . . . . . . . 35 
$\begin{array}{ll}\text { A Proving Lemma } 15 & 37\end{array}$

A.1 Handling Non Normal-Form Protocols _ . . . . . . . . . . . . . . . . . 42

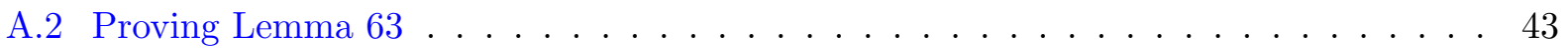

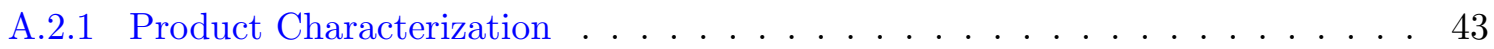

A.2.2 Graph Characterization . . . . . . . . . . . . . . . . . 43

\section{Introduction}

In the random-oracle model, the parties are given oracle access to a random function (i.e., a uniformly chosen function from the set of all functions - the all-function family), and are assumed to have unbounded computational power (though they can only make a bounded number of oracle queries). Many cryptographic primitives are known to exist in this model, such as (exponentially hard) collision resistant hash functions. More importantly, in this model it is possible to implement secure protocols for tasks that are hard to implement in the standard model, and sometimes even completely unachievable; a well known example is the work of Fiat and Shamir [7], showing how to convert three-message identification schemes to a highly efficient (non interactive) signature scheme. In the random-oracle model, their methodology preserves the security of the original scheme [20], but (for some schemes ) does not do so in the standard model [11,3].

On a different route, the random-oracle model was used to show that one cryptographic primitive cannot be used in a black-box way to construct another primitive. In their seminal work, Impagliazzo and Rudich [13] showed that no key-agreement protocol exists in the random oracle model, yielding that key-agreement cannot be black-box reduced to one-way functions. Their work has initiated a long line of follow up works (Simon [24], Gertner et al. [10], and Gennaro et al. [9], to name a few) showing that given oracle access to a certain type of function family (e.g., the family that "implements" public-key encryption) is not sufficient for building a given cryptographic primitive (e.g., oblivious transfer). Yet, the following question remained open:

What is the exact power of the random-oracle model?

Apart from being aesthetic mathematically, answers to this question are very likely to enrich our understanding of (the limitations of) black-box reductions in cryptography.

It is well known that for malicious adversaries, there exist functionalities that cannot be achieved in the information-theoretic model, i.e., where all entities are assumed to be unbounded (with no oracle access), yet can be securely computed in the random-oracle model (e.g., commitment schemes, coin-tossing protocols and, non-trivial zero-knowledge proofs). All of these functionalities, however, are blatantly trivial when considering semi-honest adversaries, which are the focus of this work.

\subsection{Our Result}

We make progress towards answering the above question, showing that, essentially, any no private input, semi-honest, two-party computation that can be securely implemented in the random-oracle model, can be securely implemented in the information-theoretic model.

Theorem 1 (Main theorem, informal). Let $\pi$ be a no private-input, m-round, $\ell$-query, oracle-aided two-party protocol. Then for any $\varepsilon>0$ there exists an $O\left(\ell^{2} / \varepsilon^{2}\right)$-query oracle-aided function Map, and a stateless, no oracle, $m$-round protocol $\widetilde{\pi}=(\widetilde{\mathrm{A}}, \widetilde{\mathrm{B}})$ such that:

$$
\operatorname{SD}\left(\left(\operatorname{out}_{\mathrm{A}}, \operatorname{out}_{\mathrm{B}}, \operatorname{Map}^{f}(\bar{t})\right)_{f \leftarrow \mathcal{F}_{\mathrm{AF}},\left(\operatorname{out}_{\mathrm{A}}, \operatorname{out}_{\mathrm{B}}, \bar{t}\right) \leftarrow\left\langle\mathrm{A}^{f}, \mathrm{~B}^{f}\right\rangle},\langle\widetilde{\mathrm{A}}, \widetilde{\mathrm{B}}\rangle\right) \in O(\varepsilon),
$$


where $\mathcal{F}_{\mathrm{AF}}$ is the all functions family, and $\langle X, Y\rangle$ stands for a random execution of the protocol $(X, Y)$, resulting in the parties' private outputs and the common transcript.

Furthermore, the projections of the above distributions to their first and third coordinates, or to their second and third coordinates are identically distributed (i.e., the transcripts concatenated with the outputs of one of the parties, are identically distributed).

Namely, the distributions induced by a random execution of $\pi^{f}$ (for a random $f \leftarrow \mathcal{F}_{\mathrm{AF}}$ ) on the parties' private outputs and the common transcript, is almost the same as that induced by a random execution of the (no oracle) protocol $\tilde{\pi}$, where the only difference is that one needs to apply an efficient procedure Map to $\pi$ 's transcript. Theorem 1 generalizes to all function families with the property that answers for distinct queries, induced by drawing a random member from the family, are independent.

A major ingredient in the proof of Theorem 1 is the dependency finder algorithm presented by Barak and Mahmoody [1], refining a similar algorithm by Impagliazzo and Rudich [13] (see Section 1.2). While we could have based the proof of Theorem 1 on a combination of several results from [1] (or alternatively, to get a somewhat weaker variant of the theorem by basing the proof on a followup result of Dachman-Soled et al. [5, Lemma 5] or of Mahmoody et al. [16, Lemma A.1]), we chose to give a new proof also for this part (modulo clearly marked parts taken from [1]). The new proof (given as part of the proof of Lemma 15) holds with respect to a larger set of function families. More significantly, it is more modular and introduces several simplifications comparing to the previous proofs.

\subsubsection{Applications}

We demonstrate the usefulness of Theorem 1 via the following three examples. The first example (reproving $[13,1]$ ) concerns the existence of key-agreement protocols in the random-oracle model. Recall that key-agreement protocols cannot be realized in the information-theoretic model. Namely, for any (no oracle) protocol $\pi$, there exists a passive (i.e., semi-honest) adversary that extracts the key from the protocol's transcript. Hence, Theorem 1 yields that key-agreement protocols cannot be realized in the random-oracle model, and thus key-agreement protocols cannot be black-box reduced to one-way functions. The actual parameters achieved by applying Theorem 1, match the optimal bound given in Barak and Mahmoody [1].

As a second more detailed example, we prove that in the random-oracle model, it is impossible for two parties to accurately approximate the inner-product function in a differentially private manner. Namely, in a way that very little information is leaked about any single bit of the input of each party to the other party. A recent result of McGregor et al. [17] shows that in the information-theoretic model, it is impossible to approximately compute the inner product function in a differentially private manner. Combining their result with Theorem 1, we obtain the following fact. ${ }^{1}$

Theorem 2 (informal). Any $\ell$-query, $\left(\ell^{2}, \alpha, \gamma\right)$-differentially private protocol, errs (with constant probability) with magnitude at least $\frac{\sqrt{n}}{\log (n) \cdot e^{\alpha}}$, in computing the inner product of two $n$-bits strings.

\footnotetext{
${ }^{1}$ We mention that the result of [17] is stated for protocol with inputs, where Theorem 1 is only applicable to no-input protocols. Indeed, a fair amount of work was needed to derive an impossibility result for no-input protocols, from the work of [17].
} 
Very informally, a protocol is $(k, \alpha, \gamma)$-differentially private, if no party, making at most $k$ queries to the oracle, learns more then $\varepsilon$ information about one of the other party's input bits, except with some small probability $\gamma$.

The above result yields the impossibility of fully black-box reducing differentially private protocols for (well) approximating two-party inner-product to the existence of one-way functions. Roughly speaking, such a fully black-box reduction is a pair of efficient oracle-aided algorithms $(\mathrm{Q}, \mathrm{R})$ such that the following hold: (1) $\mathrm{Q}^{f}$ is a good approximation protocol of the inner-product for any function $f$, and (2) $\mathrm{R}^{f, \mathcal{A}}$ inverts $f$, for any adversary $\mathcal{A}$ that learns too much about the input of one of the parties in $\mathrm{Q}^{f}$. Since a random sample from the all-function family is hard to invert (cf., $[13,9]$ ), the existence of such a reduction yields that $\mathrm{Q}^{f}$ is differentially-private with respect to poly-query adversaries, when $f$ is chosen at random from the set of all functions. ${ }^{2}$ Hence, Theorem 2 yields the following result.

Corollary 3 (informal). There exists no fully black-box reduction from $(\alpha, \gamma)$-differentially private protocol computing the inner product of two n-bit strings with error magnitude less than $\frac{\sqrt{n}}{\log (n) \cdot e^{\alpha}}$, to one-way functions.

We mention that, following an observation made by McGregor et al. [17], Theorem 2 and Corollary 3 imply similar results for two-party differentially private protocols for the Hamming distance functionality. ${ }^{3}$

The third (and last) example is with respect to no-input secure function evaluation. Let $G=$ $\left(G_{\mathrm{A}}, G_{\mathrm{B}}\right)$ be a distribution over $\mathcal{A} \times \mathcal{B}$, where $G_{\mathrm{A}}$ and $G_{\mathrm{B}}$ denote its marginal distributions over $\mathcal{A}$ and $\mathcal{B}$ respectively. A protocol $\pi=(\mathrm{A}, \mathrm{B})$ is an information-theoretically $\delta$-secure implementation of $G$, if it is a $\delta$-correct (no-oracle) implementation of $G$ (i.e., the local outputs of the parties induced by a random execution of $\pi$ are $\delta$-close to $G$ ), and is $\delta$-private according to the simulation paradigm (against all-powerful distinguishers). Specifically, there exists an algorithm (a simulator) that on input $x \in \operatorname{Supp}\left(G_{\mathrm{A}}\right)$, outputs a view for A that is $\delta$-close to the distribution of A's view, conditioned on A's local output being $x$. Similarly, there exists a simulator for B's view. A protocol $\pi$ is a $(T, \delta)$-secure random-oracle implementation of $G$, if it is a correct random-model implementation of $G$, and it is $\delta$-private according to the simulation paradigm, against $T$-query, all-powerful distinguishers. Finally, $G$ is $\delta$-trivial, if it has an information-theoretically $\delta$-secure implementation. Theorem 1 yields the following result.

Theorem 4 (informal). Let $\pi$ be an $\ell$-query oracle-aided protocol that is an $\left(O\left(\ell^{2} / \delta^{2}\right)\right.$, $\left.\delta\right)$-secure implementation of a distribution $G$ in the random-oracle model. Then, $G$ is $O(\delta)$-trivial.

Applying Theorem 4 to a distribution $G$ that has a $(\operatorname{poly}(n), 1 / \operatorname{poly}(n))$-secure random-oracle model implementation, it follows that $G$ has a $1 / \operatorname{poly}(n)$-secure no-oracle implementation. We note that Theorem 4 does not seem to imply the previous two examples. Since, for instance, the notion of differential privacy cannot be realized via the real/ideal paradigm.

\footnotetext{
${ }^{2}$ Assume towards a contradiction the existence of a poly-query adversary $\mathcal{A}$ for $\mathrm{Q}^{f}$, then the poly-query $\mathrm{R}^{f, \mathcal{A}}$ would successfully invert a random $f$.

${ }^{3}$ The inner product between two bit strings $x, y$ can be expressed as $\operatorname{IP}(x, y)=w(x)+w(y)+H_{d}(x, y)$, where the weight $w(z)$ is number of 1-bits in $z$. Thus, a differentially private protocol for estimating the Hamming distance $H_{d}(x, y)$ can be turned into one for the inner product by having the parties send differentially private approximations of the weights of their inputs.
} 


\subsection{Our Technique}

When using a no-oracle protocol to emulate an oracle-aided protocol $\pi$, having oracle access to a random member of the all-function family, the crucial issue is to find all common information the parties share at a given point. The clear obstacle are the oracle calls: the parties might share information without explicitly communicating it, say by making the same oracle call.

Here comes into play the Dependency Finder of Impagliazzo and Rudich [13], and Barak and Mahmoody [1] (algorithm Eve, in their terminology). This oracle-aided algorithm (Finder, hereafter) gets as input a communication transcript $\bar{t}$ of a random execution of $\pi$, and an oracle access to $f$, the "oracle" used by the parties in this execution. Algorithm Finder outputs a list of query/answer pairs to $f$ that with high probability contains all oracle queries that are common to both parties (and possibly also additional ones). Moreover, with high probability Finder is guaranteed not to make "too many" oracle queries.

Equipped with Finder, we give the following definition for the mapping procedure Map and the stateless (no-oracle) protocol $\widetilde{\pi}=(\widetilde{\mathrm{A}}, \widetilde{\mathrm{B}})$ : on a communication transcript $\bar{t}$, the oracle-aided algorithm Map ${ }^{f}$ outputs $\left(\left(\bar{t}_{1}, \mathcal{I}_{1}=\operatorname{Finder}^{f}\left(\bar{t}_{1}\right)\right),\left(\bar{t}_{1,2}, \mathcal{I}_{2}=\operatorname{Finder}^{f}\left(\bar{t}_{1,2}\right)\right) \ldots,\left(\bar{t}, \mathcal{I}_{m}=\operatorname{Finder}^{f}(\bar{t})\right)\right)$. Namely, Map invokes Finder on each prefix of the transcript, and outputs the result. The no-oracle protocol $\widetilde{\pi}=(\widetilde{\mathrm{A}}, \widetilde{\mathrm{B}})$ is defined as follows: assume that $\widetilde{\mathrm{A}}$ speaks in round $(i+1)$, and that the $i$ 'th message is $\left(\left(\bar{t}_{1}, \mathcal{I}_{1}\right), \ldots,\left(\bar{t}_{1, \ldots, i}, \mathcal{I}_{i}\right)\right)$. The stateless, no-oracle $\widetilde{\mathrm{A}}$ samples random values for $f \in \mathcal{F}_{\mathrm{AF}}$ and the random coins of $\mathrm{A}$, conditioned on $\left(\bar{t}_{1, \ldots, i}, \mathcal{I}_{i}\right)$ being the protocol's transcript. It then lets $t_{i+1}$ be the next message of $A$ induced by the above choice of $f$ and random coins, and sends $\left(\bar{t}^{\prime}=\left(\bar{t}_{1, \ldots, i}, t_{i+1}\right)\right.$, Finder $\left.^{f}\left(\bar{t}^{\prime}\right)\right)$ back to $\widetilde{B}$. In case this is the last round of interaction, $\widetilde{\mathrm{A}}$ locally outputs the (local) output of $A$ induced by this choice of $f$ and random coins. In other words, $\widetilde{A}$ selects a random view (including the oracle itself) for $A$ that is consistent with the information contained in the no-oracle protocol augmented transcript (i.e., the transcript of the oracle protocol and the oracle calls), and then acts as A would.

The fact that $\widetilde{A}$ perfectly emulates $A$ (and that $\widetilde{B}$ perfectly emulates $B$ ) trivially holds for information theoretic reasons. For the same reason, it also holds that the transcript generated by applying $\operatorname{Map}^{f}$ to a random transcript of $\pi^{f}$, where $f \leftarrow \mathcal{F}_{\mathrm{AF}}$, generates exactly the same transcript as a random execution of $\tilde{\pi}$ does (actually, the above facts hold for any reasonable definition of Finder ${ }^{4}$ and for any function family). The interesting part is arguing that the joint output of the no-oracle protocol has similar distribution to that of the oracle-aided protocol. To see that this is not trivial, assume that in the last round both oracle parties make the same oracle query $q$ and output the query/answer pair $(q, f(q))$. If it happens that $(q, \cdot) \notin \mathcal{I}$, where $\mathcal{I}=\operatorname{Finder}(\bar{t})$ is the query/answer pairs made by the final call to Finder on transcript $\bar{t}$, then the answer that each of the no-oracle parties compute for the query $q$ might be different. In this case, the joint output of the no-oracle protocol does not look like the joint output of the oracle protocol. Luckily, the above scenario is unlikely to happen due to the guarantee of Finder; with high probability $\mathcal{I}$ contains all common queries that the two parties made, yielding that the joint output of the no-oracle protocol has similar distribution to that of the oracle protocol. It turns out that the above example generalizes to any possible protocol, yielding that the above mapping and no-oracle protocol are indeed the desired ones.

\footnotetext{
${ }^{4}$ Whose output contains all queries it made to the oracle.
} 


\subsection{Related Work}

In their seminal work, Impagliazzo and Rudich [13] showed that there are no key-agreement protocols in the random-oracle model, and deduce that key-agreement protocols cannot be black-box reduced to one-way functions. This result was later improved by Barak and Mahmoody [1], showing there are no $\ell$-query key-agreement protocols in the random-oracle model, secure against adversary making $O\left(\ell^{2}\right)$ queries. Thus, matching the upper bound of Merkle [18].

In an independent work, Mahmoody et al. [16] show that the all-function family (and thus oneway functions) are useless for secure function evaluation of deterministic, polynomial input-domain, two-party functionalities. In other words, deterministic, bounded input domain functionalities that can be securely computed in the random-oracle model, are the trivial ones - functionalities that can be securely computed unconditionally. The comparison to the result stated here is that [16] handle with input functionalities, but only deterministic with polynomial input domain, where here we handle input-less functionalities, but including randomized ones. Putting the two results together, gives a partial characterization of the power of the random-oracle model for (semi-honest) two-party computation. It is still open, however, whether the random-oracle model is useful for securely computing randomized functionalities with inputs, or functionalities of super-polynomial input domain.

\subsubsection{Additional Black-box Separations}

Following [13], the method of black-box separation was subsequently used in many other works: [22] shows that there does not exist a black-box reduction from a $k$-pass secret key agreements to $(k-1)$-pass secret key agreements; [24] shows that there exist no black-box reductions from collision-free hash functions to one-way permutations; [14] shows that there exists no construction of one-way permutations based on one-way functions. Other works using this paradigm contain $[4,8,9,10,12,15,25]$, to name a few.

\subsubsection{Differential Privacy}

Distributed differential privacy was considered by Beimel et al. [2], who studied the setting of multiparty differentially private computation (where an $n$-bit database is shared between $n$ parties). They gave a separation between information theoretic and computational differential privacy in the distributed setting. The notion of computational differential privacy was considered in Mironov et al. [19]. They presented several definitions of computational differential privacy, studied the relationships between these definitions, and constructed efficient two-party computational differentially private protocols for approximating the Hamming-distance between two vectors. Two-party differential privacy (where an $n$-bit database is shared between two parties) was considered by McGregor et al. [17]. They prove a lower-bound on the accuracy of two party differentially private protocols, in the information theoretic model, for computing the inner-product between two $n$-bit strings (and, consequently for protocols for computing the Hamming distance). Hence, proving a separation between information theoretic and computational two-party differentially private computation. In this paper, we extend the lower-bound of [17] to the random-oracle model. 


\subsection{Open Problems}

As mentioned above, the main open problem is the full characterization of the power of the randomoracle model with respect to semi-honest adversaries. Specifically, is it possible to come up with a similar mapping from any (also with inputs) oracle-aided protocol to an equivalent one in the no-oracle model? Another interesting problem is to use our mapping (or a variant of it) to show that the random-oracle model is also useless for protocols (say, input-less) that are secure against fail-stop adversaries. An immediate implication of such a result would be that optimally-fair coin tossing are impossible to achieve in the random function model. ${ }^{5}$

\section{Paper Organization}

Formal definitions are given in Section 2. The main result of this paper is given in Section 3. Different applications of the main result are given in Section 4.

\section{Preliminaries}

\section{$2.1 \quad$ Notations}

Let poly be the set of all polynomials, and let PPTM stand for probabilistic polynomial-time algorithm. A function $\mu: \mathbb{N} \rightarrow[0,1]$ is negligible, denoted $\mu(n)=\operatorname{neg}(n)$, if $\mu(n)=n^{-\omega(1)}$. For $m \in \mathbb{N}$, let $[m]=\{1, \ldots, m\}$. For a finite set $\mathcal{S}$, we let $\chi_{\mathcal{S}}$ stand for its characteristic function, and write $x \leftarrow \mathcal{S}$ to denote that $x$ is selected according to the uniform distribution over $\mathcal{S}$. Similarly, for a random variable $X$, we write $x \leftarrow X$ to denote that $x$ is chosen according to $X$. The support of a distribution $D$ over a finite set $\mathcal{U}$, denoted $\operatorname{Supp}(D)$, is defined as $\{u \in \mathcal{U}: D(u)>0\}$. The statistical distance between two distributions $P$ and $Q$ over a finite set $\mathcal{U}$, denoted $\operatorname{SD}(P, Q)$, is defined as $\frac{1}{2} \sum_{u \in \mathcal{U}}|P(u)-Q(u)|$, and is known to be equal to $\max _{\mathcal{S} \subseteq \mathcal{U}}\left(\operatorname{Pr}_{P}[\mathcal{S}]-\operatorname{Pr}_{Q}[\mathcal{S}]\right)$. We say that two distributions $P$ and $Q$ are $\delta$-close if $\operatorname{SD}(P, Q) \leq \delta$.

\subsection{Interactive Protocols}

A two-party protocol $\pi=(\mathrm{A}, \mathrm{B})$ (with no oracle access) is a pair of probabilistic interactive Turing machines. The communication between the Turing machines $A$ and $B$ is carried out in rounds, where in each round one of the parties is active and the other party is idle. In the $j$ 'th round of the protocol, the currently active party $\mathrm{P}$ acts according to its partial view, writing some value on its output tape, and then sending a message to the other party (i.e., writing the message on the common tape).

The communication transcript (i.e., the "transcript") of a given execution of the protocol $\pi=$ $(A, B)$, is the list of messages $\bar{t}$ exchanged between the parties in an execution of the protocol, where $\bar{t}_{1, \ldots, j}$ denotes the first $j$ messages in $\bar{t}$. A view of a party contains its input, its random tape and the messages exchanged by the parties during the execution. Specifically, A's view is a tuple $v_{\mathrm{A}}=\left(i_{\mathrm{A}}, r_{\mathrm{A}}, \bar{t}\right)$, where $i_{\mathrm{A}}$ is A's input, $r_{\mathrm{A}}$ are A's coins, and $\bar{t}$ is the transcript of the execution. We let $\left(v_{\mathrm{A}}\right)_{j}$ denote the partial view of $\mathrm{A}$ in the first $j$ rounds of the execution described by $v_{\mathrm{A}}$, namely, $\left(v_{\mathrm{A}}\right)_{j}=\left(i_{\mathrm{A}}, r_{\mathrm{A}}, \bar{t}_{1, \ldots, j}\right)$; we define $v_{\mathrm{B}}$ analogously. We call $v=\left(v_{\mathrm{A}}, v_{\mathrm{B}}\right)$ the joint view of $\mathrm{A}$

\footnotetext{
${ }^{5}$ We mention that Dachman-Soled et al. [5] showed such an impossibility result for $O(n / \log n)$-round protocols, where $n$ being the random function input length.
} 
and $\mathrm{B}$, and let $v_{j}=\left(\left(v_{\mathrm{A}}\right)_{j},\left(v_{\mathrm{B}}\right)_{j}\right)$. Given a distribution (or a set) $\mathcal{D}$ on the joint views of $\mathrm{A}$ and $\mathrm{B}$, we let $\mathcal{D}_{\mathrm{A}}$ be the projection of $\mathcal{D}$ on $\mathrm{A}$ 's view (i.e., $\operatorname{Pr}_{\mathcal{D}_{\mathrm{A}}}\left[v_{\mathrm{A}}\right]=\operatorname{Pr}_{\left(v_{\mathrm{A}}, \cdot\right) \leftarrow \mathcal{D}}\left[v_{\mathrm{A}}\right]$ ), and define $\mathcal{D}_{\mathrm{B}}$ analogously. Finally, we sometimes refer to a well structured tuple $v$ as a "view" of $\pi$, even though $v$ happens with zero probability. When we wish to stress that we consider a view that has non-zero probability, we call it a valid view.

We call $\pi$ an $m$-round protocol, if for every possible random tapes for the parties, the number of rounds is exactly $m$. Given a joint view $v$ (containing the views of both parties) of an execution of $(\mathrm{A}, \mathrm{B})$ and $\mathrm{P} \in\{\mathrm{A}, \mathrm{B}\}$, let $v_{\mathrm{P}}$ denote $\mathrm{P}$ 's part in $v$ and let $\operatorname{trans}(v)$ denote the communication transcript in $v$. For $j \in[m]$, let $\operatorname{out}_{j}^{\mathrm{P}}(v)=\operatorname{out}_{j}^{\mathrm{P}}\left(v_{\mathrm{P}}\right)$ denote the output of party $\mathrm{P}$ at the end of the $j$ 'th round of $v$ (i.e., the string written on $\mathrm{P}^{\prime}$ s output tape), where $\operatorname{out}_{j}^{\mathrm{P}}(v)=\operatorname{out}_{j-1}^{\mathrm{P}}(v)$, in case $\mathrm{P}$ is inactive in the $j$ 'th round of $v$.

We sometimes consider stateless protocols - the parties hold no state, and in each round act on the message received in the previous round with freshly sampled random coins. Throughout this paper we almost solely consider no-private input protocols - the parties' only input is the common input (the only exception to that is in Section 4.2, additional required notations introduced therein). Given a no-input two-party protocol $\pi$, let $\langle\pi\rangle$ be the distribution over the joint views of the parties in a random execution of $\pi$.

\subsubsection{Oracle-Aided Protocols}

An oracle-aided, two-party protocol $\pi=(\mathrm{A}, \mathrm{B})$ is a pair of interactive Turing machines, where each party has an additional tape called the oracle tape; the Turing machine can make a query to the oracle by writing a string $q$ on its tape. It then receives a string ans (denoting the answer for this query) on the oracle tape.

For simplicity, we only consider function families whose inputs and outputs are binary strings. For an oracle-aided, no-input, two-party protocol $\pi=(\mathrm{A}, \mathrm{B})$ and a function family $\mathcal{F}$, we let $\Omega^{\mathcal{F}, \pi}$ be the set of all triplets $\left(r_{\mathrm{A}}, r_{\mathrm{B}}, f\right)$, where $r_{\mathrm{A}}$ and $r_{\mathrm{B}}$ are possible random coins for $\mathrm{A}$ and $\mathrm{B}$, and $f \in \mathcal{F}$ (henceforth, we typically omit the superscript $(\mathcal{F}, \pi)$ from the notation, whenever their values are clear from the context). For $f \in \mathcal{F}$, the distribution $\left\langle\pi^{f}=\left(\mathrm{A}^{f}, \mathrm{~B}^{f}\right)\right\rangle$, is defined analogously to $\langle\pi\rangle=\langle A, B\rangle$, i.e., it is the distribution over the joint views of parties in a random execution of $\pi$ with access to $f$. Given some information inf about some element of $\Omega$ (e.g., a set of query/answer pairs, or a view), let $\operatorname{Pr}_{\Omega}[$ inf $]=\operatorname{Pr}_{\omega \leftarrow \Omega}[\omega$ is consistent with inf $]$, and let $\operatorname{Pr}_{\Omega \mid \text { inf' }}[$ inf $]$ be this probability conditioned that $\omega$ is consistent with inf ${ }^{\prime}$ ( set to zero in case $\operatorname{Pr}_{\Omega}\left[\mathrm{inf}^{\prime}\right]=0$ ).

Given a (possibly partial) execution of $\pi^{f}$, the views of the parties contain additional lists of query/answer pairs made to the oracle throughout the execution of the protocol. Specifically, A's view is a tuple $v_{\mathrm{A}}=\left(r_{\mathrm{A}}, \bar{t}, f_{\mathrm{A}}\right)$, where $r_{\mathrm{A}}$ are A's coins, $\bar{t}$ is the transcript of the execution, and $f_{\mathrm{A}}$ are the oracle answers to A's queries. By convention, the active party in round $j$ first makes all its queries to the oracle for this round, and then writes a value to its output tape and send a message to the other party. We denote by $\left(f_{\mathrm{P}}\right)_{j}$ the oracle answers to the queries that party $\mathrm{P}$ makes during the first $j$ rounds. As above, we let $\left(v_{\mathrm{A}}\right)_{j}$ denote the partial view of $\mathrm{A}$ in the first $j$ rounds of the execution described by $v_{\mathrm{A}}$, namely, $\left(v_{\mathrm{A}}\right)_{j}=\left(r_{\mathrm{A}}, \bar{t}_{1, \ldots, j},\left(f_{\mathrm{A}}\right)_{j}\right)$. We define $v_{\mathrm{B}}$ analogously.

For $\omega \in \Omega$, we let view $(\omega)$ be the full view of the parties determined by $\omega$. We say that a "view" $v$ is consistent with $(\mathcal{F}, \pi)$, if $\operatorname{Pr}_{\Omega^{\mathcal{F}, \pi}}[v]>0$.

We assume without loss of generality that the party acting in the last round outputs a final message. Therefore, a partial transcript $\bar{t}$ of the protocol uniquely determines the length of the 
partial execution that generated it (i.e., the number of rounds of $\pi$ played), which we denote by $|\bar{t}|$. We consider the following distributions.

Definition $5(\Omega(\bar{t}, \mathcal{I})$ and $\mathcal{V} \mathcal{I E} \mathcal{W}(\bar{t}, \mathcal{I}))$. Given a partial transcript $\bar{t}$ and a set of query/answer pairs $\mathcal{I}$, let $\Omega(\bar{t}, \mathcal{I})=\Omega^{\mathcal{F}, \pi}(\bar{t}, \mathcal{I})$ be the set of all tuples $\left(r_{\mathrm{A}}, r_{\mathrm{B}}, f\right) \in \Omega=\Omega^{\mathcal{F}, \pi}$, in which $f$ is consistent with $\mathcal{I}$, and $\bar{t}$ is a prefix of the transcript induced by $\left\langle\mathrm{A}^{f}\left(r_{\mathrm{A}}\right), \mathrm{B}^{f}\left(r_{\mathrm{B}}\right)\right\rangle$. Given a set $\mathcal{P} \subseteq \Omega$, let $\Omega_{\mathcal{P}}(\bar{t}, \mathcal{I})=\Omega(\bar{t}, \mathcal{I}) \cap \mathcal{P}$.

Let $\mathcal{V} \mathcal{I E} \mathcal{W}(\bar{t}, \mathcal{I})=\mathcal{V} \mathcal{I} \mathcal{E} \mathcal{W}^{\mathcal{F}, \pi}(\bar{t}, \mathcal{I})$ be the value of $\operatorname{view}(\omega)_{|\bar{t}|}$ for $\omega \leftarrow \Omega(\bar{t}, \mathcal{I})$, and define $\mathcal{V} \mathcal{I E} \mathcal{W}_{\mathcal{P}}^{\mathcal{F}, \pi}(\bar{t}, \mathcal{I})$ analogously.

We note that since we consider the uniform distribution over $\Omega$, we have that for any partial transcript $\bar{t}$, set of query/answer pairs $\mathcal{I}$, set $\mathcal{P} \subseteq \Omega$, and information inf about some element of $\Omega$ it holds that $\operatorname{Pr}_{\Omega_{\mathcal{P}}(\bar{t}, \mathcal{I})}[$ inf $]=\operatorname{Pr}_{\Omega \mid \bar{t}, \mathcal{I}, \mathcal{P}}[$ inf $]$.

\section{Mapping Oracle-Aided Protocols to No-Oracle Protocols}

In this section we prove our main result, a mapping from protocols in the random-oracle model to (inefficient) no-oracle protocols.

\subsection{Dependent Views}

In the following we fix an $m$-round oracle-aided protocol $\pi$ and a function family $\mathcal{F}$. We would like to restrict $\mathcal{V} \mathcal{I E} \mathcal{W}(\bar{t}, \mathcal{I})$ to those views for which $\mathcal{I}$ contains all the joint information of the parties about $f$. We start by formally defining what it means for $\mathcal{I}$ to contain all the joint information.

Definition 6. Let $v_{\mathrm{A}}$ be a $j_{\mathrm{A}}$-round view for $\mathrm{A}$ and $v_{\mathrm{B}}$ be a $j_{\mathrm{B}}$-round view for $\mathrm{B}$, for some $j_{\mathrm{A}}, j_{\mathrm{B}} \in$ $[m]$. For $i \in\left[j_{\mathrm{A}}\right]$, let $\mathcal{I}_{\mathrm{A}}{ }^{i}$ be the set of query/answer pairs that $\mathrm{A}$ makes in the $i$ 'th round of $v_{\mathrm{A}}$ (where $\mathcal{I}_{\mathrm{A}}{ }^{i}=\emptyset$, if $\mathrm{A}$ is idle in round $i$ ), and define $\mathcal{I}_{\mathrm{B}}{ }^{i}$ analogously. Given a set $\mathcal{I}$ of query/answer pairs, we define

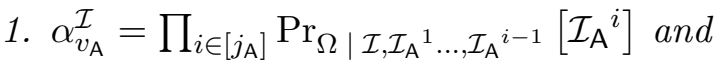

$$
\begin{aligned}
& \text { 2. } \alpha_{v_{\mathrm{A}} \mid v_{\mathrm{B}}}^{\mathcal{I}}=\prod_{i \in\left[j_{\mathrm{A}}\right]} \operatorname{Pr}_{\Omega \mid \mathcal{I}, \mathcal{I}_{\mathrm{A}}{ }^{1}, \mathcal{I}_{\mathrm{B}}{ }^{1}, \ldots, \mathcal{I}_{\mathrm{A}}{ }^{i-1}, \mathcal{I}_{\mathrm{B}}{ }^{i-1}}\left[\mathcal{I}_{\mathrm{A}}{ }^{i}\right] \text {, }
\end{aligned}
$$

and define $\alpha_{v_{\mathrm{B}} \mid v_{\mathrm{A}}}^{\mathcal{I}}$ and $\alpha_{v_{\mathrm{B}}}^{\mathcal{I}}$ analogously.

Intuitively, $\alpha_{v_{\mathrm{A}}}^{\mathcal{I}}$ is the probability of A's view of $f$ given $\mathcal{I}$, and $\alpha_{v_{\mathrm{A}} \mid v_{\mathrm{B}}}^{\mathcal{I}}$ is this probability when conditioning also on B's view. We will focus on those views with $\alpha_{v_{\mathrm{A}}}^{\mathcal{I}}=\alpha_{v_{\mathrm{A}} \mid v_{\mathrm{B}}}^{\mathcal{I}}$ and $\alpha_{v_{\mathrm{B}}}^{\mathcal{I}}=\alpha_{v_{\mathrm{B}} \mid v_{\mathrm{A}}}^{\mathcal{I}}$.

Definition 7 (dependent views). Let $v=\left(v_{\mathrm{A}}, v_{\mathrm{B}}\right)$ be a pair of (possibly partial) valid views. ${ }^{6}$ We say that $v_{\mathrm{A}}$ and $v_{\mathrm{B}}$ are dependent with respect to a set of query/answer pairs $\mathcal{I}$ and a function family $\mathcal{F}$, denoted Dependent $t_{\mathcal{I}}^{\mathcal{F}}(v)=1$, if $\alpha_{v_{\mathrm{A}}}^{\mathcal{I}} \neq \alpha_{v_{\mathrm{A}} \mid v_{\mathrm{B}}}^{\mathcal{I}}$ or $\alpha_{v_{\mathrm{B}}}^{\mathcal{I}} \neq \alpha_{v_{\mathrm{B}} \mid v_{\mathrm{A}}}^{\mathcal{I}} \cdot{ }^{7}$

A pair of views $v=\left(v_{\mathrm{A}}, v_{\mathrm{B}}\right)$ with $\operatorname{Dependent}_{\mathcal{I}}(v)=0$ is called independent. We let $\operatorname{Ind}^{\mathcal{F}, \pi}(\bar{t}, \mathcal{I})=$ $\left\{\omega \in \Omega(\bar{t}, \mathcal{I}): \operatorname{Dependent}_{\mathcal{I}}^{\mathcal{F}}\left(\operatorname{view}(\omega)_{|\bar{t}|}\right)=0\right\}$ and let $\mathcal{V} \mathcal{I} \mathcal{E} \mathcal{W}_{\text {Ind }}^{\mathcal{F}, \pi}(y)$ stand for $\mathcal{V} \mathcal{I} \mathcal{E} \mathcal{W}_{\operatorname{Ind}^{\mathcal{F}, \pi}(y)}^{\mathcal{F}}(y)$.

\footnotetext{
${ }^{6}$ While properly defined for any pair of views $\left(v_{\mathrm{A}}, v_{\mathrm{B}}\right)$, we will typically only consider the following notions for pairs with $\operatorname{trans}\left(v_{\mathrm{A}}\right)=\operatorname{trans}\left(v_{\mathrm{B}}\right)$ (i.e., both views induce the same transcript).

${ }^{7}$ One can verify that Dependent ${ }_{\mathcal{I}}^{\mathcal{F}}(v)=1$, in case $v$ is inconsistent with $\mathcal{F}$, namely, $\operatorname{Pr}_{\mathcal{F}}\left[\mathcal{I}_{\mathrm{A}}, \mathcal{I}_{\mathrm{B}}\right]=0$, where $\mathcal{I}_{\mathrm{A}}$ and $\mathcal{I}_{\mathrm{B}}$, are the lists of query/answer pairs appear in $v_{\mathrm{A}}$ and $v_{\mathrm{B}}$ respectively.
} 
The following observation (generalizing a similar observation made in [1]) plays a crucial role in the proof of our main result (stated in Section 3.3). It shows how to express the probability of a given view $v$, using $\alpha_{v_{\mathrm{A}} \mid v_{\mathrm{B}}}^{\mathcal{I}}$ and $\alpha_{v_{\mathrm{B}} \mid v_{\mathrm{A}}}^{\mathcal{I}}$. In particular, it implies that for an independent pair of views $v=\left(v_{\mathrm{B}}, v_{\mathrm{A}}\right)$ and any set $\mathcal{P} \subseteq \Omega$, the probability that $\mathcal{V} \mathcal{I} \mathcal{E} \mathcal{W}_{\mathcal{P}}(\bar{t}, \mathcal{I})=v$ can be written as a product of a term determined solely by $v_{\mathrm{A}}$ and $(\bar{t}, \mathcal{I}, \mathcal{P})$, and a term determined solely by $v_{\mathrm{B}}$ and $(\bar{t}, \mathcal{I}, \mathcal{P})$.

Proposition 8. Let $\bar{t}$ be a transcript, let $\mathcal{I}$ be a list of query/answer pairs, and let $\mathcal{P} \subseteq \Omega$. Then, for every view $v=\left(r_{\mathrm{A}}, r_{\mathrm{B}}, \cdot\right) \in \operatorname{Supp}(\mathcal{V I E \mathcal { W }}(\bar{t}, \mathcal{I}))$ with $\operatorname{Pr}_{\Omega}[v, \mathcal{I}, \bar{t}, \mathcal{P}]=\operatorname{Pr}_{\Omega}[v, \mathcal{I}]$, it holds that

$$
\operatorname{Pr}\left[\mathcal{V} \mathcal{I} \mathcal{E} \mathcal{W}_{\mathcal{P}}(\bar{t}, \mathcal{I})=v\right] \triangleq \operatorname{Pr}_{\Omega_{\mathcal{P}}(\bar{t}, \mathcal{I})}[v]=\frac{\operatorname{Pr}_{\Omega}\left[r_{\mathrm{A}}, r_{\mathrm{B}}\right] \cdot \alpha_{v_{\mathrm{A}} \mid v_{\mathrm{B}}}^{\mathcal{I}} \cdot \alpha_{v_{\mathrm{B}} \mid v_{\mathrm{A}}}^{\mathcal{I}}}{\operatorname{Pr}_{\Omega \mid \mathcal{I}}[\bar{t}, \mathcal{P}]}
$$

Proof. Note that

$$
\underset{\Omega_{\mathcal{P}}(\bar{t}, \mathcal{I})}{\operatorname{Pr}}[v]=\frac{\operatorname{Pr}_{\Omega}[v, \bar{t}, \mathcal{I}, \mathcal{P}]}{\operatorname{Pr}_{\Omega}[\bar{t}, \mathcal{I}, \mathcal{P}]}=\frac{\operatorname{Pr}_{\Omega}[v, \mathcal{I}]}{\operatorname{Pr}_{\Omega}[\bar{t}, \mathcal{I}, \mathcal{P}]}=\frac{\operatorname{Pr}_{\Omega}\left[r_{\mathrm{A}}, r_{\mathrm{B}}, \mathcal{I}\right] \cdot \operatorname{Pr}_{\Omega \mid r_{\mathrm{A}}, r_{\mathrm{B}}, \mathcal{I}}[v]}{\operatorname{Pr}_{\Omega}[\bar{t}, \mathcal{I}, \mathcal{P}]}
$$

where the second equality holds since by assumption, $\operatorname{Pr}_{\Omega}[v, \mathcal{P}, \mathcal{I}, \bar{t}]=\operatorname{Pr}_{\Omega}[v, \mathcal{I}]$. Since the choice of random coins is independent of the choice of $f$, we can write

$$
\underset{\Omega_{\mathcal{P}}(\bar{t}, \mathcal{I})}{\operatorname{Pr}}[v]=\frac{\operatorname{Pr}_{\Omega}\left[r_{\mathrm{A}}, r_{\mathrm{B}}\right] \cdot \operatorname{Pr}_{\Omega}[\mathcal{I}] \cdot \operatorname{Pr}_{\Omega \mid r_{\mathrm{A}}, r_{\mathrm{B}}, \mathcal{I}}[v]}{\operatorname{Pr}_{\Omega}[\mathcal{I}] \cdot \operatorname{Pr}_{\Omega} \mid \mathcal{I}[\bar{t}, \mathcal{P}]}=\frac{\operatorname{Pr}_{\Omega}\left[r_{\mathrm{A}}, r_{\mathrm{B}}\right] \cdot \operatorname{Pr}_{\Omega} \mid r_{\mathrm{A}}, r_{\mathrm{B}}, \mathcal{I}}{\operatorname{Pr}_{\Omega \mid \mathcal{I}}[\bar{t}, \mathcal{P}]}
$$

We next analyze the term $\operatorname{Pr}_{\Omega \mid r_{A}, r_{B}, \mathcal{I}}\left[\mathcal{I}_{A}, \mathcal{I}_{B}, \bar{t}\right]$, where $\mathcal{I}_{\mathrm{A}}$ is the set of query/answer pairs that $A$ sees according to $v_{\mathrm{A}}\left(\mathcal{I}_{\mathrm{B}}\right.$ is defined analogously). Note that this term is equal to $\operatorname{Pr}_{\Omega} \mid r_{\mathrm{A}}, r_{\mathrm{B}}, \mathcal{I}[v]$, since given $\left(r_{\mathrm{A}}, r_{\mathrm{B}}, \mathcal{I}\right)$, the values of $\left(\mathcal{I}_{\mathrm{A}}, \mathcal{I}_{\mathrm{B}}, \bar{t}\right)$ and $v$ are implied by each other.

Let $j$ be the number of rounds in $v$, and for $i \in[j]$ recall that $\mathcal{I}_{\mathrm{A}}{ }^{i}$ is the set of query/answer pairs that $\mathrm{A}$ sees in the $i$ 'th round of the execution according to $v_{\mathrm{A}}$ ( $\mathcal{I}_{\mathrm{B}}{ }^{i}$ is defined analogously). Since at any point through the execution of $\pi^{f}$, the next query of the acting party is determined by its partial view, it follows that

$$
\begin{aligned}
& \operatorname{Pr}_{\Omega \mid r_{\mathrm{A}}, r_{\mathrm{B}}, \mathcal{I}^{\prime}}\left[\mathcal{I}_{\mathrm{A}}, \mathcal{I}_{\mathrm{B}}, \bar{t}\right]=\prod_{i \in[j]} \underset{\Omega \mid r_{\mathrm{A}}, r_{\mathrm{B}}, \mathcal{I}_{,} \mathcal{I}_{\mathrm{A}}{ }^{1}, \mathcal{I}_{\mathrm{B}}{ }^{1}, \ldots, \mathcal{I}_{\mathrm{A}}{ }^{i-1}, \mathcal{I}_{\mathrm{B}}{ }^{i-1}, t_{1}, \ldots, t_{i-1}}{\operatorname{Pr}}\left[\mathcal{I}_{\mathrm{A}}^{i}, \mathcal{I}_{\mathrm{B}}{ }^{i}, t_{i}\right]
\end{aligned}
$$

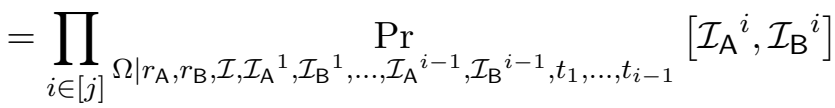

$$
\begin{aligned}
& =\prod_{i \in[j]} \operatorname{Pr}_{\Omega \mid \mathcal{I}, \mathcal{I}_{\mathrm{A}}^{1}, \mathcal{I}_{\mathrm{B}}{ }^{1}, \ldots, \mathcal{I}_{\mathrm{A}}^{i-1}, \mathcal{I}_{\mathrm{B}}{ }^{i-1}}\left[\mathcal{I}_{\mathrm{A}}^{i}, \mathcal{I}_{\mathrm{B}}{ }^{i}\right] \\
& =\left(\prod_{i \in[j]} \operatorname{Pr}_{\Omega \mid \mathcal{I}, \mathcal{I}_{\mathrm{A}}{ }^{1}, \mathcal{I}_{\mathrm{B}}{ }^{1}, \ldots, \mathcal{I}_{\mathrm{A}}{ }^{i-1}, \mathcal{I}_{\mathrm{B}}{ }^{i-1}}\left[\mathcal{I}_{\mathrm{A}}{ }^{i}\right]\right) \cdot\left(\prod_{i \in[j]} \operatorname{Pr}_{\Omega \mid \mathcal{I}^{\prime} \mathcal{I}_{\mathrm{A}}{ }^{1}, \mathcal{I}_{\mathrm{B}}{ }^{1}, \ldots, \mathcal{I}_{\mathrm{A}}{ }^{i-1}, \mathcal{I}_{\mathrm{B}}{ }^{i-1}}\left[\mathcal{I}_{\mathrm{B}}{ }^{i}\right]\right) \\
& =\alpha_{v_{\mathrm{A}} \mid v_{\mathrm{B}}}^{\mathcal{I}} \cdot \alpha_{v_{\mathrm{B}} \mid v_{\mathrm{A}}}^{\mathcal{I}}
\end{aligned}
$$

where the second equation holds since the $i$ 'th message is (deterministically) determined by the randomness of the parties, the oracle answers and the transcript till now. The third one holds since the distribution on the oracle answers at each point during the execution is a 
function of $\mathcal{I}$ and the previous queries made by the parties (recall that $\operatorname{Pr}_{\Omega \mid \text { inf }}{ }^{\prime}\left[\mathcal{I}_{\mathrm{A}}{ }^{i}, \mathcal{I}_{\mathrm{B}}{ }^{i}\right]=$ $\operatorname{Pr}_{\omega \leftarrow \Omega \mid \text { inf }^{\prime}}\left[\omega\right.$ is consistent with $\left.\mathcal{I}_{\mathrm{A}}{ }^{i}, \mathcal{I}_{\mathrm{B}}{ }^{i}\right]$ and that the first $i-1$ messages are determined by the randomness of the parties and the oracle answers to the queries made till round $i-1)$. Finally, the fourth one holds since only one party is active in each round (hence, for every $i$ either $\mathcal{I}_{\mathrm{A}}{ }^{i}$ or $\mathcal{I}_{\mathrm{B}}{ }^{i}$ is empty).

\subsection{Intersecting Views}

A special case of dependent views is when the two paries share a common oracle query not in $\mathcal{I}$.

Definition 9 (intersecting views). A (possibly partial) pair of views $v=\left(v_{\mathrm{A}}, v_{\mathrm{B}}\right)$ are intersecting with respect to a set of query/answer pairs $\mathcal{I}$, denoted $\operatorname{Intersect}_{\mathcal{I}}(v)=1$, if $v_{\mathrm{A}}$ and $v_{\mathrm{B}}$ share $a$ common query $q$ not in $\mathcal{I}($ i.e., $(q, \cdot) \notin \mathcal{I})$.

For most function families, an intersection implies being dependent (with respect to the same list of query/answer pairs). In this paper we limit our attention to "simple" function families for which also the other direction holds, namely dependency implies intersection.

Definition 10 (simple function families). A function family $\mathcal{F}$ is simple, if for any oracle-aided protocol $\pi$, list $\mathcal{I}$ of query/answer pairs that is consistent with some $f \in \mathcal{F}$, and a (possibly partial) pair of views $v=\left(v_{\mathrm{A}}, v_{\mathrm{B}}\right)$ consistent with $\mathcal{I}$, it holds that $\operatorname{Dependent~}_{\mathcal{I}}^{\mathcal{F}}(v)=1$ iff $\operatorname{Intersect}_{\mathcal{I}}(v)=1$.

It is not hard to verify that the family of all functions is simple (see Lemma 55 for the formal proof).

\subsection{Oracle-Aided to No-Oracle Protocol Mapping}

The following theorem shows that an execution of an oracle-aided protocol with oracle access to a random $f \in \mathcal{F}$, where $\mathcal{F}$ is a simple function family, can be mapped to an execution of a related protocol with no oracle access. In Section 4 we use this result to prove limitations on the power of oracle-aided protocols in achieving specific cryptographic tasks.

Definition 11 (oracle-aided to no-oracle mapping). A pair of a function family $\mathcal{F}$ and a no-input, $m$-round, oracle-aided protocol $\pi=(\mathrm{A}, \mathrm{B})$, has a $(T, \varepsilon)$-mapping, if there exists a deterministic, oracle-aided T-query algorithm Map and a stateless, $m$-round, no-input (and no-oracle) protocol $(\widetilde{\mathrm{A}}, \widetilde{\mathrm{B}})$, such that the following hold:

1. $\mathrm{SD}\left(\mathcal{D}_{\mathcal{F}}, \mathcal{D}_{P}\right) \leq \varepsilon$ for every $j \in[m]$, where

$$
\begin{aligned}
& \mathcal{D}_{\mathcal{F}}=\left(\operatorname{out}_{j}^{\mathrm{A}}(v), \operatorname{out}_{j}^{\mathrm{B}}(v), \operatorname{Map}^{f}\left(\operatorname{trans}(v)_{1, \ldots, j}\right)\right)_{f \leftarrow \mathcal{F}, v \leftarrow\left\langle\mathrm{A}^{f}, \mathrm{~B}^{f}\right\rangle} \quad \text { and }, \\
& \mathcal{D}_{P}=\left(\operatorname{out}_{j}^{\widetilde{\mathrm{A}}}(v), \operatorname{out}_{j}^{\widetilde{\mathrm{B}}}(v), \operatorname{trans}(v)_{1, \ldots, j}\right)_{v \leftarrow\langle\widetilde{\mathrm{A}}, \widetilde{\mathrm{B}}\rangle} \cdot{ }^{8}
\end{aligned}
$$

Furthermore, $\mathcal{D}_{P}[1,3] \equiv \mathcal{D}_{\mathcal{F}}[1,3]$ and $\mathcal{D}_{P}[2,3] \equiv \mathcal{D}_{\mathcal{F}}[2,3] .{ }^{9}$

\footnotetext{
${ }^{9}$ I.e., the projections of $\mathcal{D}_{P}$ and $\mathcal{D}_{\mathcal{F}}$ to their transcript part and the output of one of the parties, are identically distributed.
} 
2. For every $f \in \mathcal{F}$, an m-round transcript $\bar{t}$ and $j \in[m]$, it holds that $\operatorname{Map}^{f}\left(\bar{t}_{1, \ldots, j}\right)=$ $\operatorname{Map}^{f}(\bar{t})_{1, \ldots, j}$. Furthermore, the oracle calls made in $\operatorname{Map}^{f}\left(\bar{t}_{1, \ldots, j}\right)$ are a subset of those made in $\operatorname{Map}^{f}(\bar{t})$.

Theorem 12. Let $\mathcal{F}$ be a simple function family and let $\pi=(\mathrm{A}, \mathrm{B})$ be an $\ell$-query, oracle-aided, no-input protocol, then $(\mathcal{F}, \pi)$ has an $\left(256 \cdot \ell^{2} / \varepsilon^{2}, \varepsilon\right)$-mapping for any $0<\varepsilon \leq 1$.

Remark 13 (Round complexity of the no-oracle protocol). The proof of Theorem 12 can be easily modified to yield a one-message no-oracle protocol (in this case, $\mathcal{D}_{\mathcal{F}}$ and $\mathcal{D}_{\mathcal{P}}$ should be modified to reflect the transcript and outputs at the end of the executions). The roles of $\widetilde{\mathrm{A}}$ and $\widetilde{\mathrm{B}}$ in the resulting protocol however, cannot reflect as closely the roles of $\mathrm{A}$ and $\mathrm{B}$, as done in the many-round, nooracle protocol stated above.

The heart of the proof is the following lemma, proof given in Appendix A. ${ }^{10}$

Definition 14 (DependencyFinder). Let $\mathcal{F}$ be a function family and let $\pi=(\mathrm{A}, \mathrm{B})$ be an m-round oracle-aided protocol. A deterministic oracle-aided algorithm Finder is a $(T, \varepsilon)$-DependencyFinder for $(\mathcal{F}, \pi)$ if the following holds for any $j \in[m]$ : consider the following random process $\mathrm{CF}=$ $\mathrm{CF}(\mathcal{F}, \pi$, Finder $)$ :

1. Choose $\left(r_{\mathrm{A}}, r_{\mathrm{B}}, f\right) \leftarrow \Omega^{\mathcal{F}, \pi}$ and let $\bar{t}$ be the $j$-round transcript of $\pi$ induced by $\left(r_{\mathrm{A}}, r_{\mathrm{B}}, f\right)$.

2. For $i=1$ to $j$ set $\mathcal{I}_{i}=\mathcal{I}_{i-1} \cup \operatorname{Finder}^{f}\left(\bar{t}_{1, \ldots, i}, \mathcal{I}_{i-1}\right)$ (letting $\left.\mathcal{I}_{0}=\emptyset\right)$, where Finder $^{f}(x)$ is the set of queries/answers made by $\operatorname{Finder}^{f}(x)$ to $f$.

3. Output $\left(\bar{t}, \mathcal{I}_{j}\right)$.

Then

1. $\mathrm{E}_{d \leftarrow \mathrm{CF}}\left[\mathrm{SD}\left(\mathcal{V I E} \mathcal{W}^{\mathcal{F}, \pi}(d),\left(\mathcal{V I E} \mathcal{W}^{\mathcal{F}, \pi}(d)_{\mathrm{A}}, \mathcal{V} \mathcal{I} \mathcal{E} \mathcal{W}^{\mathcal{F}, \pi}(d)_{\mathrm{B}}\right)\right)\right] \leq \varepsilon$, and

2. $\operatorname{Pr}[\#$ of $f$-calls made in $\mathrm{CF}>T] \leq \varepsilon$.

That is, conditioned on a random transcript of $\pi^{\mathcal{F}}$ and the oracle queries made by a $(T, \delta)$ DependencyFinder, the parties' views are close to being in a product distribution.

Lemma 15. Let $\mathcal{F}$ be a simple function family and let $\pi=(\mathrm{A}, \mathrm{B})$ be an $\ell$-query oracle-aided protocol, then $(\mathcal{F}, \pi)$ has a $\left(64 / \delta^{2}, \ell \delta\right)$-DependencyFinder for any $0<\delta \leq 1 / \ell$.

We now use Lemma 15 to prove Theorem 12.

\subsubsection{Proving Theorem 12}

Fix a simple function family $\mathcal{F}$ and a no-input, $m$-round, $\ell$-query oracle-aided protocol $\pi$. Fix $0<\varepsilon \leq 1$ and let Finder be the $\left(T=256 \cdot \ell^{2} / \varepsilon^{2}, \varepsilon / 2\right)$-DependencyFinder guaranteed by Lemma 15 for $(\mathcal{F}, \pi)$ (taking $\delta=\varepsilon / 2 \ell$ ). We start by defining the mapping algorithm and then we define a protocol with no oracle access.

\footnotetext{
${ }^{10}$ As mentioned in the introduction, the proof of Lemma 15 could be essentially derived by combining several statements in [1]. Alternatively, a somewhat weaker variant of the lemma can be directly proved using the followup result of Dachman-Soled et al. [5, Lemma 5] or of Mahmoody et al. [16, Lemma A.1].
} 


\section{Algorithm 16 (Map).}

Oracle: $f \in \mathcal{F}$.

Input: $j$-round transcript $\bar{t}$ of $\pi$.

Operation:

1. For $i=1$ to $j$ set $\mathcal{I}_{i}=\mathcal{I}_{i-1} \cup \operatorname{Finder}^{f}\left(\bar{t}_{1, \ldots, i}, \mathcal{I}_{i-1}\right)$ (letting $\left.\mathcal{I}_{0}=\emptyset\right)$.

2. If in some round $i^{*}$ the overall number of $f$ calls (made by Finder) is $T$, halt the above procedure and set $\mathcal{I}_{i^{*}}$ to be the set of $T$ query/answer pairs obtained so far, ${ }^{11}$ and set $\mathcal{I}_{i}=\mathcal{I}_{i^{*}}$ for all $i^{*}<i \leq j$.

3. Output $\left(\bar{t}_{1}, \mathcal{I}_{1}\right),\left(\bar{t}_{1,2}, \mathcal{I}_{2}\right), \ldots,\left(\bar{t}, \mathcal{I}_{j}\right)$.

The no-oracle protocol. Our stateless, no-oracle protocol $\widetilde{\pi}=(\widetilde{\mathrm{A}}, \widetilde{\mathrm{B}})$, emulates the oracle-aided protocol $\pi$ by keeping the "important" oracle queries as part of the transcript, and selecting the rest of the oracle at random (independently in each round). In particular, $\widetilde{A}$ is active in $\widetilde{\pi}$ in the same rounds that $A$ is in $\pi$ (same for $\widetilde{B}$ and $B$ ). The definition of $\widetilde{A}$ is given below $(\widetilde{B}$ is analogously defined).

Algorithm $17(\widetilde{\mathrm{A}})$.

Input: A pair $(\bar{t}, \mathcal{I})$, where $\bar{t}$ is a transcript of length $j$ and $\mathcal{I}$ is a set of query/answer pairs.

Operation:

1. Sample $\left(r_{\mathrm{A}}, r_{\mathrm{B}}, f\right) \leftarrow \Omega(\bar{t}, \mathcal{I})$, and let out $_{j+1}$ and $t_{j+1}$ denote $\mathrm{A}$ 's output and message respectively, in the $(j+1)$ round of $\left\langle\mathrm{A}^{f}\left(r_{\mathrm{A}}\right), \mathrm{B}^{f}\left(r_{\mathrm{B}}\right)\right\rangle$.

2. Output out ${ }_{j+1}$.

3. Compute the value of $\mathcal{I}_{j+1}$ output by $\operatorname{Map}^{f}\left(\overline{t_{j+1}}\right)$ for $\overline{t_{j+1}}=\left(\bar{t}, t_{j+1}\right)$.

4. Send $\left(\overline{t_{j+1}}, \mathcal{I}_{j+1}\right)$ to $\widetilde{\mathrm{B}}$.

Using Lemma 15, one can prove that above mapping function and no-oracle protocol, indeed establish mapping and protocol guaranteed in Theorem 12.

Proof of Theorem 12. We prove that algorithm Map (Algorithm 16) and protocol $\widetilde{\pi}=(\widetilde{\mathrm{A}}, \widetilde{\mathrm{B}})(\mathrm{Al}-$ gorithm 17), form an $(T, \varepsilon)$-mapping for $(\mathcal{F}, \pi)$. By construction, algorithm Map is deterministic (since Finder is deterministic), makes at most $T$ queries and fulfills the second item of Definition 11. Towards showing that (Map, $\widetilde{\pi}$ ) fulfills also the first property of Definition 11 with respect to stated parameter, we prove the following claim:

Claim 18. $\left(\operatorname{Map}^{f}\left(\operatorname{trans}(v)_{1, \ldots, j}\right)\right)_{f \leftarrow \mathcal{F}, v \leftarrow\left\langle\mathrm{A}^{f}, \mathrm{~B}^{f}\right\rangle} \equiv\left(\operatorname{trans}(v)_{1, \ldots, j}\right)_{v \leftarrow\langle\widetilde{\mathrm{A}}, \widetilde{\mathrm{B}}\rangle}$ for every $j \in[m]$.

\footnotetext{
${ }^{11}$ I.e., augmenting $\mathcal{I}_{i^{*}-1}$ with the queries/answers made in round $i^{*}$ before halting.
} 
Proof. We prove the claim by induction on $j$; by the induction hypothesis and the fact that $\operatorname{Map}^{f}\left(\operatorname{trans}(v)_{1, \ldots, j}\right)_{1, \ldots, j-1}=\operatorname{Map}^{f}\left(\operatorname{trans}(v)_{1, \ldots, j-1}\right)$ (since Map fulfills the second item of Definition 11), it suffices to prove that the distributions in the claim are the same, conditioned that their $(j-1)$ - "round" prefix is fixed to some value $\left(\ldots,\left(\bar{t}_{1, \ldots, j-1}, \mathcal{I}_{j-1}\right)\right)$. Since $\mathcal{I}_{j-1}$ is the set of queries/answers made by $\operatorname{Map}^{f}\left(\operatorname{trans}(v)_{1, \ldots, j-1}\right)$ to $f$, the value of the right-hand-side distribution under this conditioning is $\operatorname{Map}^{f}\left(\bar{t}^{\prime}\right)$, where $f$ and $\bar{t}^{\prime}$ are the function and the $j$-round transcript of $\pi$ respectively, induced by $\omega \leftarrow \Omega\left(\bar{t}_{1, \ldots, j-1}, \mathcal{I}_{j-1}\right)$. It is easy to verify that, under this conditioning, the latter process also describes the left-hand-side distribution.

We next note that Claim 18 yields that $\mathcal{D}_{P}[1,3] \equiv \mathcal{D}_{\mathcal{F}}[1,3]$ (and similarly that $\mathcal{D}_{P}[2,3] \equiv$ $\left.\mathcal{D}_{\mathcal{F}}[2,3]\right)$; indeed, conditioned on $\mathcal{D}_{P}[3]=\mathcal{D}_{\mathcal{F}}[3]=\left(\ldots,\left(\bar{t}_{1, \ldots, j}, \mathcal{I}_{j}\right)\right)$, the values of both $\mathcal{D}_{P}[1]$ and of $\mathcal{D}_{\mathcal{F}}$ [1] (i.e., A's output), are obtained by the following random process: sample $\omega \leftarrow \Omega\left(\bar{t}_{1, \ldots, j}, \mathcal{I}_{j}\right)$ and output A's output in the $j$ 'th round induced by $\omega$.

Finally, the definition of $\widetilde{\pi}=(\widetilde{A}, \widetilde{B})$ yields that

$$
\begin{aligned}
\mathcal{D}_{P} & :=\left(\operatorname{out}_{j}^{\widetilde{\mathrm{A}}}(v), \operatorname{out}_{j}^{\widetilde{\mathrm{B}}}(v), \operatorname{trans}(v)_{1, \ldots, j}\right)_{v \leftarrow\langle\widetilde{\mathrm{A}}, \widetilde{\mathrm{B}}\rangle} \\
& \equiv\left(\operatorname{out}_{j}^{\mathrm{A}}\left(v_{\mathrm{A}}\right), \operatorname{out}_{j}^{\mathrm{B}}\left(v_{\mathrm{B}}\right), \tilde{t}_{1, \ldots, j}\right)_{\tilde{t} \leftarrow \operatorname{trans}(\langle\widetilde{\mathrm{A}}, \widetilde{\mathrm{B}}\rangle), v_{\mathrm{A}} \leftarrow \mathcal{V} \mathcal{I E} \mathcal{V}\left(\tilde{t}_{j}\right)_{\mathrm{A}}, v_{\mathrm{B}} \leftarrow \mathcal{V I E} \mathcal{I}\left(\tilde{t}_{j}\right)_{\mathrm{A}}}
\end{aligned}
$$

where we recall that $\tilde{t}_{j}$ consists of a pair $\left(\overline{t_{j}}, \mathcal{I}_{j}\right)$. It is easy to verify that

$$
\begin{aligned}
\mathcal{D}_{\mathcal{F}} & :=\left(\operatorname{out}_{j}^{\mathrm{A}}(v), \operatorname{out}_{j}^{\mathrm{B}}(v), \operatorname{Map}^{f}\left(\operatorname{trans}(v)_{1, \ldots, j}\right)\right)_{f \leftarrow \mathcal{F}, v \leftarrow\left\langle\mathrm{A}^{f}, \mathrm{~B}^{f}\right\rangle} \\
& \equiv\left(\operatorname{out}_{j}^{\mathrm{A}}\left(v_{\mathrm{A}}\right), \operatorname{out}_{j}^{\mathrm{B}}\left(v_{\mathrm{B}}\right), \tilde{t}_{1, \ldots, j}\right)_{f \leftarrow \mathcal{F}, \bar{t} \leftarrow \operatorname{trans}\left(\left\langle\mathrm{A}^{f}, \mathrm{~B}^{f}\right\rangle\right), \tilde{t} \leftarrow \operatorname{Map}^{f}(\bar{t}), v \leftarrow \mathcal{V} \mathcal{I E} \mathcal{W}\left(\tilde{t}_{j}\right)}
\end{aligned}
$$

and therefore Claim 18 yields that

$$
\mathcal{D}_{\mathcal{F}} \equiv\left(\operatorname{out}_{j}^{\mathrm{A}}\left(v_{\mathrm{A}}\right), \operatorname{out}_{j}^{\mathrm{B}}\left(v_{\mathrm{B}}\right), \tilde{t}_{1, \ldots, j}\right)_{\tilde{t} \leftarrow \operatorname{trans}(\langle\widetilde{\mathrm{A}}, \widetilde{\mathrm{B}}\rangle), v \leftarrow \mathcal{V} \mathcal{I E} \mathcal{W}\left(\tilde{t}_{j}\right)}
$$

We conclude the proof using the fact that Finder is a $(T, \varepsilon / 2)$-DependencyFinder for $(\mathcal{F}, \pi)$. The issue to note is that Process CF (described in Definition 14) may make arbitrary number of oracle queries, while Map is restricted to at most $T$ queries. Let $\mathcal{S}$ be the set of pairs $d=\left(\bar{t}_{1, \ldots, j}, \mathcal{I}_{j}\right)$ in the support of the Process CF with $\left|\mathcal{I}_{j}\right| \leq T$. Note that the probability that CF outputs $d \in \mathcal{S}$, is exactly the probability of the transcript part being of the form $(\ldots, d)$ according to distribution $\mathcal{D}_{\mathcal{F}}$, where by Claim 18 this is also the probability of the this event according to $\mathcal{D}_{P}$. We bound the statistical distance between $\mathcal{D}_{\mathcal{F}}$ and $\mathcal{D}_{P}$, by separately bounding the part contributed by transcripts $(\ldots, d)$ with $d \in \mathcal{S}$ and by transcripts $(\ldots, d)$ with $d \notin \mathcal{S}$.

The fact that Finder is a $(T, \varepsilon / 2)$-DependencyFinder for $(\mathcal{F}, \pi)$, yields a bound of $\varepsilon / 2$ on the contribution of elements whose transcripts is inside $\mathcal{S}$ to the statistical distance between $\mathcal{D}_{\mathcal{F}}$ and $\mathcal{D}_{P}$. It also bounds by $\varepsilon / 2$ the probability that CF outputs elements whose transcripts is outside $\mathcal{S}$; yielding the same bound on the contribution of such elements to the statistical distance between $\mathcal{D}_{\mathcal{F}}$ and $\mathcal{D}_{P}$. We conclude that $\operatorname{SD}\left(\mathcal{D}_{\mathcal{F}}, \mathcal{D}_{P}\right) \leq \varepsilon / 2+\varepsilon / 2=\varepsilon$. 


\section{Applications}

In this section we use our main result (i.e., the oracle-aided to no-oracle protocol mapping for simple function families) from Section 3 to derive the impossibility of realizing three cryptographic tasks, with respect to simple function families (yielding the same results with respect to the all function family, which is shown to be simple in Section 4.4.2). In Section 4.1 we re-establish the result of [13], showing that key-agreement protocols cannot be realized with respect to simple function families. Then, in Section 4.2, we extend the lower-bound of [17] on the accuracy of two-party differentially private no-oracle protocols, to show it also holds (with a slight loss in parameters) for oracle-aided differentially private protocols (with respect to simple function families). Finally, in Section 4.3, we show that no-input functionalities that cannot be securely evaluated in the no-oracle model (even when allowing some small loss of security), cannot be securely evaluated (again, even with some small loss of security) by oracle-aided protocols that are given access to a random member of a simple function family. In Section 4.4, we use the fact that the all-function family is simple to prove the impossibility of reducing the above first two primitives to the hardness of one-way functions in a black-box manner. ${ }^{12}$

Remark 19 (definitions for no-oracle primitives). Throughout this section we only give formal definitions (of the security and correctness) of primitives with respect to oracle-aided protocols. Deriving formal definitions for their no-oracle counterparts can be easily done by considering the trivial function family (i.e., the singleton family, whose only member returns $\perp$ on any query).

\subsection{Key Agreement Protocols}

In a key-agreement protocol two parties wish to agree on a common secret in a secure way an observer (adversary) seeing the communication transcript, cannot find the secret. Below we prove that with respect to a certain class of function families, non-trivial key-agreement cannot be achieved. We start by formally defining the notion of key agreement. We then recall the known fact that in the no-oracle model, an adversary can reveal any secret agreement between two parties in the strongest possible sense (i.e., with the same probability that the parties themselves agree). Combining this fact with the mapping from oracle-aided to no-oracle protocols, described in Section 3, yields a similar result for oracle-aided protocols (formally stated in Section 4.1.2).

We remark that the results presented in this section yield very little conceptual added-value to what was already shown by $[13,1]$. We do, however, present them here to demonstrate how they are easily derived from our main result (Theorem 12), and as a warm-up before moving on to the other applications of our main result, described in Sections 4.2 and 4.3.

\subsubsection{Standard Definitions and Known Facts}

Recall (see Section 2.2) that for a joint view $v \in \operatorname{Supp}\left(\left\langle\pi^{f}\right\rangle\right)$, we let $\operatorname{trans}(v)$ denote the communication transcript in $v$, and $\operatorname{out}_{i}^{\mathrm{P}}(v)$ denote the output of the party $\mathrm{P}$ at the $i$ 'th round. In the following we let $\operatorname{out}^{\mathrm{P}}(v)=\operatorname{out}_{m}^{\mathrm{P}}(v)$, where $m$ is the last round in $v$.

Definition 20 (key agreement protocol). Let $0 \leq \gamma, \alpha \leq 1$ and $k \in \mathbb{N}$. A two-party, oracle-aided protocol $\pi=(\mathrm{A}, \mathrm{B})$ is a $(k, \alpha, \gamma)$-key-agreement protocol with respect to a function family $\mathcal{F}$, if the following hold:

\footnotetext{
${ }^{12}$ It is possible to obtain a similar result for no-input secure function evaluation, however, we will not include this result here.
} 
Consistency: $\pi$ is $(1-\alpha)$-consistent with respect to $\mathcal{F}$. Namely for every $f \in \mathcal{F}$,

$$
\operatorname{Pr}_{v \leftarrow\left\langle\pi^{f}\right\rangle}\left[\operatorname{out}^{\mathrm{A}}(v)=\operatorname{out}^{\mathrm{B}}(v)\right] \geq 1-\alpha .
$$

Security: For every $P \in\{\mathrm{A}, \mathrm{B}\}$ and any $k$-query adversary Eve,

$$
\underset{f \leftarrow \mathcal{F}, v \leftarrow\left\langle\pi^{f}\right\rangle}{\operatorname{Pr}}\left[\operatorname{Eve}^{f}(\operatorname{trans}(v))=\operatorname{out}^{P}(v)\right] \leq \gamma .
$$

A protocol $\pi$ is an $(\alpha, \gamma)$-key-agreement protocol, if it is a $(\cdot, \alpha, \gamma)$-key-agreement protocol with respect to the trivial function family. ${ }^{13}$

In the no-oracle model, all correlation between the parties is implied by the transcript. Hence, an adversary that on a given transcript $\bar{t}$ samples a random view for $\mathrm{A}$ that is consistent with $\bar{t}$ and outputs whatever $A$ would upon this view, agrees with B with the same probability as does $A$. This simple argument yields the following fact.

Fact 21. Let $0 \leq \alpha \leq 1$ and let $\pi=(\mathrm{A}, \mathrm{B})$ be a no-oracle, two-party, no-input protocol. Assume that the probability that in a random execution of $\pi$ both parties output the same value is $1-\alpha$. Then there exists an adversary that, given the transcript of a random execution of $\pi$, outputs the same value as does $\mathrm{B}$ with probability $1-\alpha$.

An immediate implication of Fact 21 is that there does not exist any no-oracle, two-party, $(\alpha, \gamma)$-key-agreement protocol for any $0 \leq \gamma<1-\alpha$. We next use our main result from Section 3 to prove a similar result for oracle-aided protocols.

\subsubsection{Limits on Oracle-Aided Key-Agreement Protocols}

In the language of the above definition, our main result is stated as follows.

Theorem 22. Let $\mathcal{F}$ be a function family and let $\pi$ be an oracle-aided protocol. Assume that the pair $(\mathcal{F}, \pi)$ has a $(T, \varepsilon)$-mapping, then $\pi$ is not a $(T, \alpha, \gamma)$-key-agreement with respect to $\mathcal{F}$ for any $0 \leq \gamma<1-(\alpha+\varepsilon)$.

Proof. Assume to the contrary that $\pi$ is a $(T, \alpha, \gamma)$-key-agreement with respect to $\mathcal{F}$ for some $0 \leq \gamma<1-(\alpha+\varepsilon)$. Let $\widetilde{\pi}=(\widetilde{\mathrm{A}}, \widetilde{\mathrm{B}})$ and Map be the no-input no-oracle protocol and oracle-aided algorithm, guaranteed by the assumption of the theorem. The first item in Definition 11 yields that

$$
\operatorname{SD}\left(\left(\operatorname{out}^{\widetilde{A}}(v), \operatorname{out}^{\widetilde{B}}(v)\right)_{v \leftarrow\langle\widetilde{\pi}\rangle},\left(\operatorname{out}^{\mathrm{A}}(v), \operatorname{out}^{\mathrm{B}}(v)\right)_{f \leftarrow \mathcal{F}, v \leftarrow\left\langle\pi^{f}\right\rangle}\right) \leq \varepsilon
$$

Hence, the $(1-\alpha)$-consistency of $\pi$ yields that

$$
\tau:=\operatorname{Pr}_{v \leftarrow\langle\widetilde{\pi}\rangle}\left[\operatorname{out}^{\widetilde{A}}(v)=\operatorname{out}^{\widetilde{B}}(v)\right] \geq 1-(\alpha+\varepsilon) .
$$

\footnotetext{
${ }^{13}$ We remark that our impossibility result (as well the results of $[13,1]$ ) would also hold with respesct to a weaker definition, requiring consistency to hold for a random $f$, rather than for every $f \in \mathcal{F}$.
} 
Fact 21 yields an adversary $\widetilde{\text { Eve }}$ that given the transcript of a random execution of $\widetilde{\pi}$, outputs the same value as does B with probability $\tau$. Let Eve be an adversary for $\pi$ that upon a transcript $\bar{t}$ (of

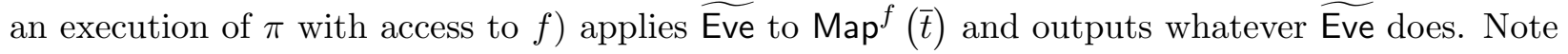
that by Definition 11, Eve makes at most $T$ oracle calls. The definition of Eve yields that

$$
\begin{aligned}
& \operatorname{Pr}_{f \leftarrow \mathcal{F}, v \leftarrow\left\langle\pi^{f}\right\rangle}\left[\operatorname{Eve}^{f}(\operatorname{trans}(v))=\operatorname{out}^{\mathrm{B}}(v)\right] \\
& =\underset{f \leftarrow \mathcal{F}, v \leftarrow\left\langle\pi^{f}\right\rangle}{\operatorname{Pr}}\left[\widetilde{\operatorname{Eve}}\left(\operatorname{Map}^{f}(\operatorname{trans}(v))\right)=\operatorname{out}^{\mathrm{B}}(v)\right] \\
& =\underset{\tilde{v} \leftarrow\langle\tilde{\pi}\rangle}{\operatorname{Pr}}\left[\widetilde{\operatorname{Eve}}(\operatorname{trans}(\tilde{v}))=\operatorname{out}^{\widetilde{B}}(\tilde{v})\right]=\tau \geq 1-(\alpha+\varepsilon),
\end{aligned}
$$

where the second equality follows from the furthermore statement of the first item in Definition 11, stating that $\left(\operatorname{Map}^{f}(\operatorname{trans}(v))\right.$, out $\left.{ }^{\mathrm{B}}(v)\right)$ is identically distributed as $\left(\operatorname{trans}(\tilde{v}), \operatorname{out}^{\widetilde{B}}(\tilde{v})\right)$, where $f$, $v$, and $\tilde{v}$ are sampled as in Equation (8).

Combining Theorems 12 and 22 yields the following result.

Theorem 23. Let $\mathcal{F}$ be a simple function family. For parameters $k, \ell \in \mathbb{N}$ and $\alpha, \gamma \in \mathbb{R}$ with $k \geq 2^{10} \cdot\left(\frac{\ell}{1-\alpha-\gamma}\right)^{2}$ and $1-\alpha>\gamma \geq 0$, there exists no $\ell$-query oracle-aided protocol, that is $(k, \alpha, \gamma)$-key-agreement with respect to $\mathcal{F}$.

Proof. Let $\mathcal{F}$ be a simple function family and let $\pi$ be an $\ell$-query oracle-aided protocol. For $\varepsilon=\frac{1-\alpha-\gamma}{2}$, Theorem 12 yields that $(\mathcal{F}, \pi)$ has a $(T, \varepsilon)$-mapping for $T=256 \cdot\left(\frac{\ell}{\varepsilon}\right)^{2}=2^{10} \cdot\left(\frac{\ell}{1-\alpha-\gamma}\right)^{2}$. Since $0 \leq \gamma<1-(\alpha+\varepsilon)$ and $k \geq T$, Theorem 22 yields that $\pi$ is not a $(k, \alpha, \gamma)$-key-agreement protocol with respect to $\mathcal{F}$.

\subsection{Differentially Private Two-Party Computation}

In this section we apply our main result to extend the lower-bound of McGregor et al. [17] to oracle-aided protocols equipped with simple function families. Specifically, we show that when given access to a random member of a simple function family (e.g., the all-function family), any twoparty, differentially private, oracle-aided protocol computing the inner product of two $s$-bit strings, exhibits error magnitude of roughly $\Omega(\sqrt{s} / \log s)$ (see Section 4.2.2 for the formal statement). This fact is later used in Section 4.4.4 to show that differentially private accurate computation of the inner product, cannot be reduced to one-way functions in a black-box way.

Unlike the case of key-agreement protocols discussed in Section 4.1, here we consider a setting where the parties do hold private inputs. Since our main result (Theorem 12) only handles no input protocols, in order to apply it to differentially private protocols we need first to reduce the question in hand to such no-input protocols. Indeed, much of the following text deals with this transformation.

In proving the results of this section, we begin (Section 4.2.3) by using Theorem 12 together with an ("information theoretic") result by [17], to show that a "sampled-input" protocol cannot be both differentially private and a good approximation for the inner product of two strings. In a sampled-input protocol, the no-input parties choose the inputs to the functionality (in our case, the inner product function) by themselves. We then (Section 4.2.4) derive the same limitation on 
protocols with inputs, but where the correctness and privacy are measured with respect to uniformly chosen inputs. Finally (Section 4.2.5), we use the latter result to show the same limitation for fixed inputs, hence proving our main result. Before starting with the aforementioned plan, we first recall the formal definition of differential privacy, cite the result of [17] (Section 4.2.1), and formally state our main results (Section 4.2.2).

\subsubsection{Standard Definitions and Known Facts}

We start by recalling the standard definition of differential privacy for mechanisms (in a centralized model, where the mechanism has access to all the data). Let $\Sigma$ be some alphabet. For strings $x, x^{\prime} \in \Sigma^{s}$, let $H_{d}\left(x, x^{\prime}\right)=\left|\left\{i \in[s]: x_{i} \neq x_{i}^{\prime}\right\}\right|$ denote the Hamming distance between $x$ and $x^{\prime}$. A randomized mechanism operating on $s$-long strings (databases) is a randomized algorithm that given input in $\Sigma^{s}$, outputs a value in the range $\mathcal{R}$.

Definition $24((\alpha, \gamma)$-differential privacy [6] (in the centralized model)). A randomized mechanism $\mathrm{M}$ over $\Sigma^{s}$ is $(\alpha, \gamma)$-differentially private, if for every distinguisher $\mathrm{D}$ and every $x, x^{\prime} \in \Sigma^{s}$ with $H_{d}\left(x, x^{\prime}\right)=1$, it holds that

$$
\operatorname{Pr}[\mathrm{D}(\mathrm{M}(x))=1] \leq e^{\alpha} \cdot \operatorname{Pr}\left[\mathrm{D}\left(\mathrm{M}\left(x^{\prime}\right)\right)=1\right]+\gamma .
$$

If $\mathrm{M}$ satisfies $(\alpha, \gamma)$-differential privacy with $\gamma=0$, then $\mathrm{M}$ is just $\alpha$-differentially private. ${ }^{14}$

Differential privacy extends naturally to the setting of two-party (semi-honest) protocols by requiring that the view of each party satisfies differential privacy with respect to the other party's private input. In this work we use a relaxed definition (and hence potentially easier to achieve) that only requires that the communication transcript (rather than the whole view of a party) is differentially private with respect to each party's input. Such a requirement is easily implied by the above requirement on views, since any distinguisher that breaks the privacy seeing only the transcript can break the privacy seeing the whole view of a party (by simply disregarding everything in the view but the transcript part). We next define differential privacy for protocols using similar definitions to those given in $[2,17,19]$. Indeed, our definitions are close in spirit to the definition of IND-CDP from [19] (which they showed to be implied by all other definitions that they considered for computational differential privacy).

In the following, when we say protocol, we mean a two-party protocol. We focus on protocols where each party holds an $s$-bit string as its private input, and call such protocols $s$-bit input protocols. We adapt the notations from Section 2.2 (defined for no-input protocols) to protocols with inputs, with the understanding that the view of a party also includes its $s$-bit private input. Specifically, given an oracle-aided protocol $\pi=(\mathrm{A}, \mathrm{B})$, a function $f$, and $x, y \in\{0,1\}^{*}$, we define $\left\langle\pi^{f}(x, y)\right\rangle$ to be $\left\langle\left(\mathrm{A}^{f}(x), \mathrm{B}^{f}(y)\right)\right\rangle$ (i.e., the distribution over the joint views of parties in a random execution of $\pi$ with access to $f$, where the private input of $\mathrm{A}$ is $x$ and the private input of $\mathrm{B}$ is $y)$. Recall that for $v \in \operatorname{Supp}\left(\left\langle\pi^{f}(x, y)\right\rangle\right)$, we let $\operatorname{trans}(v)$ denote the communication transcript in $v$, and we let $\operatorname{out}_{i}^{\mathrm{P}}(v)$ denote the output of the party $\mathrm{P}$ at the $i$ 'th round. In the following we let $\operatorname{out}^{\mathrm{P}}(v)$ denote the output of the party $\mathrm{P}$ at the last round of $v$.

Definition 25 (differential privacy for oracle-aided protocols). Let $\mathcal{F}$ be a function family and let $\pi=(\mathrm{A}, \mathrm{B})$ be an s-bit input, oracle-aided protocol. The protocol $\pi$ is $(k, \alpha, \gamma)$-differentially private

\footnotetext{
${ }^{14}$ Throughout this section, we assume $\alpha, \gamma \geq 0$.
} 
with respect to $\mathcal{F}$ and $\mathrm{A}$, if for every $k$-query, oracle-aided distinguisher $\mathrm{D}$ and every $x, x^{\prime}, y \in\{0,1\}^{s}$ with $H_{d}\left(x, x^{\prime}\right)=1$, it holds that

$$
\operatorname{Pr}_{f \leftarrow \mathcal{F}, v \leftarrow\left\langle\pi^{f}(x, y)\right\rangle}\left[\mathrm{D}^{f}(\operatorname{trans}(v))=1\right] \leq e^{\alpha} \cdot \underset{f \leftarrow \mathcal{F}, v \leftarrow\left\langle\pi^{f}\left(x^{\prime}, y\right)\right\rangle}{\operatorname{Pr}}\left[\mathrm{D}^{f}(\operatorname{trans}(v))=1\right]+\gamma .
$$

Being $(k, \alpha, \gamma)$-differentially private with respect to $\mathcal{F}$ and $\mathrm{B}$, is analogously defined. If $\pi$ is $(k, \alpha, \gamma)$ differentially private with respect to $\mathcal{F}$ and both parties, then it is $(k, \alpha, \gamma)$-differentially private with respect to $\mathcal{F}$.

Finally, $\pi$ is $(\alpha, \gamma)$-differentially private, if it is $(\cdot, \alpha, \gamma)$-differentially private with respect to the trivial function family.

Note that for no-oracle protocols, the above definition of $(\alpha, \gamma)$-differentially private matches the standard (no-oracle) definition (slightly relaxed, as we only require the transcript to preserve the privacy of the parties). Our impossibility results, given below, apply to privacy parameter $\alpha$ being smaller than some constant.

Since differentially private mechanisms cannot be deterministic, for any deterministic (nonconstant) function $g$ of the input, one can only hope for the output of the mechanism being a good approximation for $g$. We next define a notion of accuracy for differentially private protocols.

Definition 26 (good approximations). Let $g:\{0,1\}^{s} \times\{0,1\}^{s} \mapsto \mathbb{R}$ be a deterministic function and let $\pi=(\mathrm{A}, \mathrm{B})$ be an s-bit input, oracle-aided protocol. The protocol $\pi$ is a $(\beta, d)$-approximation for $g$ with respect to a function family $\mathcal{F}$, if for very $f \in \mathcal{F}$, for every $x, y \in\{0,1\}^{s}$ and $\mathrm{P} \in\{\mathrm{A}, \mathrm{B}\}$, it holds that

$$
\operatorname{Pr}_{v \leftarrow\left\langle\pi^{f}(x, y)\right\rangle}\left[\left|g(x, y)-\operatorname{out}^{\mathrm{P}}(v)\right|>d\right]<\beta .
$$

Namely, we require that the output of both parties is within distance $d$ from $g(x, y)$ with probability at least $\beta$.

For two $s$-bit strings $x$ and $y$, let $\operatorname{IP}(x, y)$ denote the inner product of $x$ and $y$; that is $\operatorname{IP}(x, y)=$ $\sum_{i \in[s]} x_{i} \cdot y_{i}$. McGregor et al. [17] showed that for small enough $\gamma$, no two-party, no-oracle, $(\alpha, \gamma)$ differentially private protocol for computing the inner product of two $s$-bit databases can be a $(0.01, d)$-approximation for $d \in o(\sqrt{s} / \log s)$. This follows from the following general theorem.

Theorem 27 ([17, Theorem A.5]). Let $\pi=(\mathrm{A}, \mathrm{B})$ be an s-bit (no-oracle) protocol, let $X_{\mathrm{In}}$ and $Y_{\mathrm{In}}$ be the inputs of $\mathrm{A}$ and $\mathrm{B}$, respectively, and let $X_{\text {out }}$ and $Y_{\text {out }}$ be the outputs of $\mathrm{A}$ and $\mathrm{B}$, respectively, induced by a random execution of $\pi$. Assume that both $X_{\mathrm{In}}$ and $Y_{\mathrm{In}}$ are independently and uniformly chosen from $\{0,1\}^{s}$, and that $\pi$ is $(\alpha, \gamma)$-differentially private, then

$$
\operatorname{Pr}\left[\left|Y_{\text {out }}-\operatorname{IP}\left(X_{\text {In }}, Y_{\text {In }}\right)\right|<\Delta:=\Omega\left(\frac{\sqrt{s}}{\log s} \cdot \frac{\tau}{e^{\alpha}}\right)\right] \leq \tau
$$

for every $1 \geq \tau \geq 48 s \gamma .{ }^{15}$ The same holds for $X_{\text {out }}$.

In the next section we use similar arguments to the ones used by McGregor et al. [17], to prove a variant of Theorem 27 for (no-oracle) no-input protocols (which we call here sampled-input protocols). For that we recall a few definitions and results from [17].

\footnotetext{
${ }^{15}$ This constraint implies that $\gamma$ should be smaller than the inverse of some polynomial in $s$, however, this is how we typically think of $\gamma$.
} 
Lemma 28 ([17, Lemma A.3]). Let $\mathrm{M}$ be an $(\alpha, \gamma)$-differentially private mechanism over $\{0,1\}^{s}$. Then for every $\nu>0$ and every $x, x^{\prime} \in\{0,1\}^{s}$ with $H_{d}\left(x, x^{\prime}\right)=1$, it holds that

$$
\operatorname{Pr}_{m \leftarrow \mathrm{M}(x)}\left[\frac{\operatorname{Pr}[\mathrm{M}(x)=m]}{\operatorname{Pr}\left[\mathrm{M}\left(x^{\prime}\right)=m\right]} \notin\left[e^{-(\nu+\alpha)}, e^{(\nu+\alpha)}\right]\right]<\gamma \cdot \frac{1+e^{-(\nu+\alpha)}}{1-e^{-\nu}} .
$$

Unpredictability of bit sources. The model of random sources introduced by Santha and Vazirani [23] is one where each bit is somewhat unpredictable given the previous ones. An unpredictable $s$-bit source is a random variable over $\{0,1\}^{s}$ with the property that given any prefix of it, it is hard to guess the value of the next bit.

Definition $29\left((\eta, \gamma)\right.$-unpredictable bit source). For $\eta \in[0,1]$ a random variable $X=\left(X_{1}, \ldots, X_{s}\right)$ taking values in $\{0,1\}^{s}$ is an $(\eta, \gamma)$-unpredictable bit source, if with probability at least $1-\gamma$ over $i \leftarrow[s]$ and over $\left(x_{1}, \ldots, x_{i-1}\right) \leftarrow\left(X_{1}, \ldots, X_{i-1}\right)$, it holds that

$$
\eta \leq \frac{\operatorname{Pr}\left[X_{i}=0 \mid X_{1}=x_{1}, \ldots X_{i-1}=x_{i-1}\right]}{\operatorname{Pr}\left[X_{i}=1 \mid X_{1}=x_{1}, \ldots X_{i-1}=x_{i-1}\right]} \leq 1 / \eta .
$$

$A$ variable $X$ is $\eta$-unpredictable, if it is $(\eta, 0)$-unpredictable.

A random variable $X=\left(X_{1}, \ldots, X_{s}\right)$ taking values in $\{0,1\}^{s}$ is an $(\eta, \gamma)$-strongly-unpredictable bit source, if with probability at least $1-\gamma$ over $i \leftarrow[s]$ and over $\left(x_{1}, \ldots, x_{i-1}, x_{i+1}, \ldots, x_{s}\right) \leftarrow$ $\left(X_{1}, \ldots, X_{i-1}, X_{i+1}, \ldots, X_{s}\right)$, it holds that

$$
\eta \leq \frac{\operatorname{Pr}\left[X_{i}=0 \mid X_{1}=x_{1}, \ldots X_{i-1}=x_{i-1}, X_{i+1}=x_{i+1}, \ldots, X_{s}=x_{s}\right]}{\operatorname{Pr}\left[X_{i}=1 \mid X_{1}=x_{1}, \ldots X_{i-1}=x_{i-1}, X_{i+1}=x_{i+1}, \ldots, X_{s}=x_{s}\right]} \leq 1 / \eta .
$$

Note that if $X$ is $\eta$-unpredictable for $\eta=1$, then it is uniform. More generally, the larger $\eta$ is, the more "randomness" is the source guaranteed to have. Specifically, an unpredictable source has high min-entropy.

Fact 30. Let $X=\left(X_{1}, \ldots, X_{s}\right)$ be an $\eta$-unpredictable source, then the min-entropy of $X$, defined as $\mathrm{H}_{\infty}(X)=\min _{x \in \operatorname{Supp}(X)} \log \frac{1}{\operatorname{Pr}[X=x]}$, is at least $\beta$ s for $\beta=\log (1+\eta)$.

Proof. Fix $\left(x_{1}, \ldots, x_{s}\right) \in \operatorname{Supp}(X), i \in[s]$ and $b \in\{0,1\}$. Definition 29 yields that $\operatorname{Pr}\left[X_{i}=b \mid X_{1}=x_{1}, \ldots X_{i-1}=x_{i-1}\right] \geq \eta \cdot \operatorname{Pr}\left[X_{i}=1-b \mid X_{1}=x_{1}, \ldots X_{i-1}=x_{i-1}\right] .{ }^{16} \quad$ Since $\operatorname{Pr}\left[X_{i}=b \mid X_{1}=x_{1}, \ldots X_{i-1}=x_{i-1}\right]+\operatorname{Pr}\left[X_{i}=1-b \mid X_{1}=x_{1}, \ldots X_{i-1}=x_{i-1}\right]=1$, it follows that $(1+\eta) \cdot \operatorname{Pr}\left[X_{i}=b \mid X_{1}=x_{1}, \ldots X_{i-1}=x_{i-1}\right] \leq 1$, and therefore $\operatorname{Pr}\left[X=\left(x_{1}, \ldots, x_{s}\right)\right] \leq$ $\left(\frac{1}{1+\eta}\right)^{s}$.

We will make use of the following results from [17].

Lemma 31 ([17, Lemma A.2]). Let $X=\left(X_{1}, \ldots, X_{s}\right)$ be an $(\eta, \gamma)$-strongly-unpredictable bit source, then, for every $\nu>0$, it is $\frac{s \gamma}{\nu}$-close to some $\hat{\eta}$-unpredictable bit source, where $\hat{\eta}=\eta \cdot \frac{1-\nu}{1+\nu}$.

Corollary 32. Let $X=\left(X_{1}, \ldots, X_{s}\right)$ be an $(\eta, \gamma)$-strongly-unpredictable bit source, then it is $2 s \gamma$ close to some $\eta / 3$-unpredictable bit source.

Proof. Apply Lemma 31 with $\nu=1 / 2$.

\footnotetext{
${ }^{16}$ For $b=1$, this is implied by the right hand side inequality in the condition of Definition 29 .
} 
Theorem 33 ([17, Theorem 3.4]). Let $X$ and $Y$ be s-bit independent bit sources, where $X$ is $\eta$ unpredictable and $Y$ has min-entropy at least $\beta$ s, and let $Z=\operatorname{IP}(X, Y) \bmod m$ for some $m \in \mathbb{N}$. Assume that $s \geq c \cdot \frac{m^{2}}{\eta \beta} \cdot \log \left(\frac{m}{\beta}\right) \cdot \log \left(\frac{m}{\gamma}\right)$ for some $\gamma \in[0,1]$, where $c$ is a universal constant, then $\mathrm{SD}\left((Y, Z),\left(Y, U_{m}\right)\right) \leq \gamma$, where $U_{m}$ is uniform on $\mathbb{Z}_{m}$ and independent of $Y$.

\subsubsection{Limits on Differentially Private Oracle-Aided Protocols for Computing Inner Product}

In this section, we state our main impossibility results for differentially private, oracle-aided protocols for accurately approximating the inner-product of two $s$-bit strings. We first give (Theorem 35) a lower-bound on the accuracy of differentially private protocols for approximating the inner-product of two $s$-bit strings with respect to general function-families (for protocols that have a certain type of mapping to no-oracle protocols). We then give lower-bound on the accuracy of any differentially private, oracle-aided protocol for approximating the inner-product of two $s$-bit strings with respect to simple function families (Theorem 36).

Since these results deal with with-input protocols and since our discussion in Section 3 only handles no-input protocols, our proof proceeds by reducing the problem of with-input protocols that accurately approximate the inner-product function to a similar problem on no-input protocols. Specifically, for a given with-input protocol we consider its no-input variant (called the sampledinput variant), in which the parties use the first $s$ bits in their random input string as inputs (see the formal definition below). The proof of Theorem 35 is given in Section 4.2.5 (impossibility results for sampled-input protocols are given in Section 4.2.3).

Definition 34 (the sampled-input variant $\mu(\pi)$ ). Given an s-bit input, (possibly, oracle-aided) protocol $\pi=(\mathrm{A}, \mathrm{B})$, let $\mu(\pi)=(\mu(\mathrm{A}), \mu(\mathrm{B}))$ denote the following s-bit sampled-input protocol:

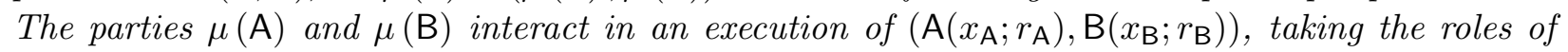
$\mathrm{A}$ and $\mathrm{B}$ respectively, where $x_{\mathrm{A}}$ [resp., $x_{\mathrm{B}}$ ] is the first $s$ bits of $\mu(\mathrm{A})$ 's [resp., $\mu(\mathrm{B})$ 's] coins, and $r_{\mathrm{A}}$ [resp., $r_{\mathrm{B}}$ ] is the rest of $\mu(\mathrm{A})$ 's [resp., $\mu(\mathrm{B})$ 's] coins. Let $a$ and $b$ be the outputs of $\mathrm{A}$ and $\mathrm{B}$, respectively, in this execution, then the outputs of $\mu(\mathrm{A})$ and $\mu(\mathrm{B})$ will be $\left(x_{\mathrm{A}}, a\right)$ and $\left(x_{\mathrm{B}}, b\right)$, respectively.

Theorem 35. For numbers $\nu>0$ and $\alpha \geq 0$, there exist numbers $\lambda>0$ and $z \in \mathbb{N}$ such that the following holds. Let $\mathcal{F}$ be a function family and let $\pi=(\mathrm{A}, \mathrm{B})$ be an oracle-aided, $s$-bit input protocol.

Assume that $\pi$ is $(T, \alpha, \gamma)$-differentially private with respect to $\mathcal{F}$, that the pair $(\mathcal{F}, \mu(\pi))$ has $a(T, \varepsilon)$-mapping (where $\mu(\pi)$ is sampled-input variant of $\pi$ ), and that $s \geq z$. Then, for some $f \in \mathcal{F},{ }^{17}$ and every $\mathrm{P} \in\{\mathrm{A}, \mathrm{B}\}$, there exist $x, y \in\{0,1\}^{\text {s }}$ such that

$$
\operatorname{Pr}_{v \leftarrow\left\langle\pi^{f}(x, y)\right\rangle}\left[\left|\operatorname{out}^{\mathrm{P}}(v)-\operatorname{IP}(x, y)\right| \leq \Delta:=\lambda \cdot \frac{\sqrt{s}}{\log s} \cdot(\tau-\varepsilon)\right] \leq \tau
$$

for every $\tau \leq 1$ with $\tau-\varepsilon \geq \max \{48 s \gamma, \nu\}{ }^{15}$

Combining Theorems 12 and 35 yields the following result.

\footnotetext{
${ }^{17}$ Actually, the following holds for most elements of $\mathcal{F}$.
} 
Theorem 36. Let $\mathcal{F}$ be a simple function family. For numbers $0<\nu<1$ and $\alpha \geq 0$, there exist numbers $\lambda>0$ and $z \in \mathbb{N}$ such that, for $s \geq z$, the following holds. Assume that $\pi$ is an s-bit input, $\ell$-query oracle-aided protocol that is $(k, \alpha, \gamma)$-differentially private with respect to $\mathcal{F}$, with $k>2^{10} \cdot\left(\frac{\ell}{1-\nu}\right)^{2}$ and $\gamma \leq \frac{\nu}{48 \cdot s}$. Then, $\pi$ is not a $(\beta, d)$-approximation with respect to $\mathcal{F}$ for the inner-product function, with $\beta<\frac{1-\nu}{2}$ and $d \leq \lambda \cdot \nu \cdot \frac{\sqrt{s}}{\log s}$.

Proof. For numbers $0<\nu<1$ and $\alpha \geq 0$, let $\lambda$ and $z$ be as in Theorem 35. Let $\mathcal{F}$ be a simple function family and let $\pi$ be an $s$-bit input, $\ell$-query oracle-aided protocol. Let $\mu(\pi)$ be the (oracle-aided) sampled-input variant of $\pi$ (see Definition 34). By construction, $\mu(\pi)$ is an $\ell$-query, oracle-aided, no-input protocol. Finally, let $\varepsilon=\frac{1-\nu}{2}$.

Theorem 12 yields that $(\mathcal{F}, \mu(\pi))$ has a $(T, \varepsilon)$-mapping for $T=256 \cdot\left(\frac{\ell}{\varepsilon}\right)^{2}=2^{10} \cdot\left(\frac{\ell}{1-\nu}\right)^{2}$.

Let $\gamma$ be such that $\gamma \leq \frac{\nu}{48 \cdot s}$. Taking $\tau=\nu+\varepsilon$, it follows that $\tau-\varepsilon \geq \max \{48 s \gamma, \nu\}$. Hence for $k \geq T$, Theorem 35 yields that if $\pi$ is $(k, \alpha, \gamma)$-differentially private with respect to $\mathcal{F}$, then it is not a $(\beta, d)$-approximation for the inner-product function with respect to $\mathcal{F}$, whenever $d \leq \lambda \cdot \frac{\sqrt{s}}{\log s} \cdot(\tau-\varepsilon)=\lambda \cdot \nu \cdot \frac{\sqrt{s}}{\log s}$ and $\beta \leq 1-\tau=1-\nu-\varepsilon$. Plugging in the value of $\varepsilon$, the latter holds whenever $\beta \leq \frac{1-\nu}{2}$.

\subsubsection{Limits on Sampled-Input Protocols}

In this section we give a lower-bound on the accuracy of no-input, two-party, differentially private protocols, where the inputs for the functionally are derived from the parties' private coins (while preserving differential privacy with respect to these inputs). We do so by combining a result from [17] (stated here as Theorem 27) and our main result from Section 3 (Theorem 12).

Definition 37 (sampled-input protocols). A no-input protocol $\pi=(\mathrm{A}, \mathrm{B})$ is an $s$-bit sampled-input protocol, if the output of party $\mathrm{A}$ in any execution of $\pi$ is of the form $(x, a)$ and the output of party $\mathrm{B}$ is of the form $(y, b)$, where $x, y \in\{0,1\}^{s}$. We call $x$ [resp., $y$ ] the sampled input of A [resp., B], and a [resp., b] the actual output of A [resp., B].

For $v \in \operatorname{Supp}\left(\left\langle\pi^{f}\right\rangle\right)$, let $\operatorname{SInp}^{\mathrm{P}}(v)$ denote the sampled input of party $\mathrm{P}$ in $v$, and $\operatorname{AOut}^{\mathrm{P}}(v)$ denote the actual output of the party $\mathrm{P} .{ }^{18}$

We next extend the notion of good-approximations to sampled-input protocols. Intuitively, we require the actual outputs of both parties to be within distance $d$ from the value of $g$ applied to the sampled inputs of the parties, except with probability $\beta$.

Definition 38 (sampled-input good approximations). Let $g:\{0,1\}^{s} \times\{0,1\}^{s} \mapsto \mathbb{R}$ be a deterministic function, and let $\pi=(\mathrm{A}, \mathrm{B})$ be an oracle-aided, s-bit sampled-input protocol. The protocol $\pi$ is a $(\beta, d)$-SI-approximation for $g$ with respect to a function family $\mathcal{F}$ and $\mathrm{P} \in\{\mathrm{A}, \mathrm{B}\}$, if for every $f \in \mathcal{F}$, it holds that

$$
\operatorname{Pr}_{v \leftarrow\left\langle\pi^{f}\right\rangle}\left[\left|g\left(\operatorname{SInp}^{\mathbf{A}}(v), \operatorname{SInp}^{\mathrm{B}}(v)\right)-\operatorname{AOut}^{\mathrm{P}}(v)\right|>d\right]<\beta .
$$

Protocol $\pi$ is a $(\beta, d)$-SI-approximation for $g$ with respect to $\mathcal{F}$, if it is a $(\beta, d)$-SI-approximation for $g$ with respect to $\mathcal{F}$ and both parties.

\footnotetext{
${ }^{18}$ Namely, $\operatorname{SInp}^{\mathrm{P}}(v)=\operatorname{out}^{\mathrm{P}}(v)_{1, \ldots, s}$ and $\operatorname{AOut}^{\mathrm{P}}(v)=\operatorname{out}^{\mathrm{P}}(v)_{s+1, \ldots}$.
} 
We also extend the notion of differential privacy to sampled-input protocols.

Definition 39 (differential privacy sampled-input protocols). Let $\mathcal{F}$ be a function family and let $\pi=(\mathrm{A}, \mathrm{B})$ be an oracle-aided, $s$-bit sampled-input protocol. The protocol $\pi$ is $(k, \alpha, \gamma)$-differentially private with respect to $\mathcal{F}$ and $\mathrm{A}$, if for every $k$-query, oracle-aided distinguisher $\mathrm{D}$ and every $x, x^{\prime} \in$ $\{0,1\}^{s}$ with $H_{d}\left(x, x^{\prime}\right)=1$, it holds that

$$
\begin{aligned}
\operatorname{Pr}_{f \leftarrow \mathcal{F}, v \leftarrow\left\langle\pi^{f}\right\rangle}\left[\mathrm{D}^{f}(\operatorname{trans}(v))=1 \mid \operatorname{SInp}^{\mathrm{A}}(v)=x\right] \\
\leq e^{\alpha} \cdot \underset{f \leftarrow \mathcal{F}, v \leftarrow\left\langle\pi^{f}\right\rangle}{\operatorname{Pr}}\left[\mathrm{D}^{f}(\operatorname{trans}(v))=1 \mid \operatorname{SInp}^{\mathrm{A}}(v)=x^{\prime}\right]+\gamma .
\end{aligned}
$$

The differential privacy of $\pi$ with respect to $(\mathcal{F}$ and) $\mathrm{B}$ is defined analogously.

The protocol $\pi$ is $(k, \alpha, \gamma)$-differentially private with respect to $\mathcal{F}$, if it is $(k, \alpha, \gamma)$-differentially private with respect to $\mathcal{F}$ and both parties.

Lower bound for no-oracle sampled-input protocols. The following theorem is a variant of Theorem 27, suited for no-oracle, sampled-input protocols.

Theorem 40. For numbers $\nu>0$ and $\alpha \geq 0$, there exist numbers $\lambda>0$ and $z \in \mathbb{N}$ such that the following holds. Let $\pi=(\mathrm{A}, \mathrm{B})$ be a no-oracle, s-bit sampled-input protocol, let $X_{\mathrm{In}}$ and $Y_{\mathrm{In}}$ be the sampled inputs of $\mathrm{A}$ and $\mathrm{B}$, respectively, and let $X_{\mathrm{out}}$ and $Y_{\mathrm{out}}$ be the actual outputs of $\mathrm{A}$ and $\mathrm{B}$, respectively, induced by a random execution of $\pi$.

Assume that both $X_{\text {In }}$ and $Y_{\text {In }}$ are uniformly distributed over $\{0,1\}^{s}$, that $\pi$ is $(\alpha, \gamma)$-differentially private and that $s \geq z$, then

$$
\operatorname{Pr}\left[\left|Y_{\text {out }}-\operatorname{IP}\left(X_{\text {In }}, Y_{\text {In }}\right)\right| \leq \Delta:=\lambda \cdot \frac{\sqrt{s}}{\log s} \cdot \tau\right] \leq \tau
$$

for every $1 \geq \tau \geq \max \{48 s \gamma, \nu\} .{ }^{15}$ The same holds for $X_{\text {out }}$.

The main difference between Theorem 40 and Theorem 27, is that Theorem 40 allows $X_{\text {In }}$ and $Y_{\text {In }}$ to be chosen during the protocol (and hence not necessarily be independent), where Theorem 27 assumes that the inputs are selected by an external entity (hence, needing to require independence of inputs). We observe that the proof of Theorem 27 given in [17], does not require a priori independence between $X_{\mathrm{In}}$ and $Y_{\mathrm{In}}$, but only that they are independent given any transcript of the protocol. The latter holds, however, for any joint distribution for $\left(X_{\mathrm{In}}, Y_{\mathrm{In}}\right)$, since the views of the parties (in the no-oracle model with no inputs) are always independent of each other, given the transcript. Indeed, Theorem 40 easily follows by slight adaptation to the proof of Theorem 27, given in [17]. For completeness, however, we include a proof (much of which, taken verbatim from $[17])$.

Let us first describe the outline of the proof given in [17] for Theorem 27 (Theorem A.5 in [17]), which is in turn the scheme of our proof. Their proof is twofold. The first part of it is a result about unpredictable bit sources, showing that it is possible to extract a uniform element in $\mathbb{Z}_{m}$ from the inner product between two independent unpredictable $s$-bit variables (even given one of these variables), provided that $m$ is somewhat less than $\sqrt{s}$. We can use this result without reproving it (for the formal statement see Theorem 33). The second part of the proof deals with 
executions of $(\alpha, \gamma)$-differentially private protocols, where the inputs of the parties are selected uniformly at random. It is shown that the input of each party in such executions, given the transcript of the execution, is close to an unpredictable bit-source. We reprove this part, with respect to sampled-input protocols. Finally, combining the above two results yields that every twoparty differentially-private protocol for approximating the inner product function must incur an error of roughly $m \approx \sqrt{s}$. Indeed, if a significantly better approximation could be computed given the transcript (and one party's input), then the inner product would be concentrated in an interval of size significantly smaller than $m$, contradicting the fact that it reduces to an almost-uniform element of $\mathbb{Z}_{m}$.

Proof of Theorem 40. Let $\pi=(\mathrm{A}, \mathrm{B})$ be a no-oracle, $s$-bit sampled-input protocol, let $X_{\text {In }}$ and $Y_{\text {In }}$ be the sampled inputs of $\mathrm{A}$ and $\mathrm{B}$, respectively, and let $X_{\text {out }}$ and $Y_{\text {out }}$ be the actual outputs of $\mathrm{A}$ and $\mathrm{B}$, respectively, induced by a random execution of $\pi$. Let $\bar{T}$ be the communication transcript in a random execution of $\pi$, and for $\bar{t} \in \operatorname{Supp}(\bar{T})$ let $X_{\operatorname{In} \mid \bar{t}}\left[\right.$ resp., $\left.Y_{\text {In } \mid \bar{t}}\right]$ be the value of $X_{\text {In }}$ [resp., $\left.Y_{\text {In }}\right]$ in such a random execution, conditioned on $\bar{T}=\bar{t}$. Assume that both $X_{\text {In }}$ and $Y_{\text {In }}$ are uniformly distributed over $\{0,1\}^{s}$ and that $\pi$ is $(\alpha, \gamma)$-differentially private. Let $\eta=e^{-(1.1+\alpha)} / 3$ and $\beta=\log (1+\eta))$. Finally, fix $\nu>0$ and $\tau \geq \max \{48 s \gamma, \nu\}$.

The proof is carried via the following claims (proofs given below). In Claim 41 we show that by the differential privacy of $\pi$, it holds that $X_{\operatorname{In} \mid \bar{t}}$ and $Y_{\operatorname{In} \mid \bar{t}}$ are, on average, close to being unpredictable. In Claim 42 we define the constants $\lambda$ and $z$ so that we can apply Theorem 33 with respect to such sources.

Claim 41. There are numbers $\left\{\gamma_{\bar{t}}\right\}_{\bar{t} \in \operatorname{Supp}(\bar{T})}$ with $\mathrm{E}\left[\gamma_{\bar{T}}\right] \leq 4 \gamma$, such that the following holds for every $\bar{t} \in \operatorname{Supp}(\bar{T}):$ the random variable $X_{\text {In } \mid \bar{t}}$ [resp., $\left.Y_{\text {In } \mid \bar{t}}\right]$ is $2 s \gamma_{\bar{t}}$-close to some $\eta$-unpredictable bit-source $\widehat{X}_{\bar{t}}$ [resp., $\widehat{Y}_{\bar{t}}$ ].

Claim 42. There are numbers $\lambda>0$ and $z \in \mathbb{N}$, functions of $\alpha$ and $\nu$, such that the following holds. Let $\Delta=\lambda \cdot \frac{\sqrt{s}}{\log s} \cdot \tau$, let $m=6 \cdot \Delta / \tau$ and let $c$ be the constant from Theorem 33. Assuming $s \geq z$, then $s \geq c \cdot \frac{m^{2}}{\eta \beta} \cdot \log \left(\frac{m}{\beta}\right) \cdot \log \left(\frac{m}{\tau / 3}\right)$.

We use the above claims for proving the proposition for $Y_{\text {out }}$, where the proof for $X_{\text {out }}$ is analogous. Fix for a moment $\bar{t} \in \operatorname{Supp}(\bar{T})$, and note that $X_{\mathrm{In} \mid \bar{t}}$ and $Y_{\mathrm{In} \mid \bar{t}}$ are independent (since $\pi$ is a no-input, no-oracle protocol). Let $\left\{\gamma_{\bar{t}}\right\}_{\bar{t} \in \operatorname{Supp}(\bar{T})}, \lambda, z, \Delta$ and $m$, be the numbers from Claims 41 and 42 . Claim 41 yields that

$$
\operatorname{SD}\left(\left(Y_{\text {In } \mid \bar{t}}, \operatorname{IP}\left(X_{\text {In } \mid \bar{t}}, Y_{\text {In } \mid \bar{t}}\right) \bmod m\right),\left(\widehat{Y}_{\bar{t}}, \operatorname{IP}\left(\widehat{X}_{\bar{t}}, \widehat{Y}_{\bar{t}}\right) \bmod m\right)\right) \leq 4 s \gamma_{\bar{t}}
$$

for some two (independent) $\eta$-unpredictable bit sources $\widehat{X}_{\bar{t}}$ and $\widehat{Y}_{\bar{t}}$. Note that by Fact 30 , both $\widehat{X}_{\bar{t}}$ and $\widehat{Y}_{\bar{t}}$ have min-entropy $\beta s$.

Assume $s \geq z$. Since by Claim $42 s \geq c \cdot \frac{m^{2}}{\eta \beta} \cdot \log \left(\frac{m}{\beta}\right) \cdot \log \left(\frac{m}{\tau / 3}\right)$, Theorem 33 yields that

$$
\operatorname{SD}\left(\left(\widehat{Y}_{\bar{t}}, \operatorname{IP}\left(\widehat{X}_{\bar{t}}, \widehat{Y}_{\bar{t}}\right) \bmod m\right),\left(\widehat{Y}_{\bar{t}}, U_{m}\right)\right) \leq \tau / 3
$$

where $U_{m}$ is independently and uniformly distributed over $\mathbb{Z}_{m}$. Finally, combining Equations (13) and (14) yields that

$$
\operatorname{SD}\left(\left(Y_{\text {In } \mid \bar{t}}, \operatorname{IP}\left(X_{\text {In } \mid \bar{t}}, Y_{\text {In } \mid \bar{t}}\right) \bmod m\right),\left(\widehat{Y}_{\bar{t}}, U_{m}\right)\right) \leq 4 s \gamma_{\bar{t}}+\tau / 3
$$


for every $\bar{t} \in \operatorname{Supp}(\bar{T})$.

In the following we assume without loss of generality that B's output is a deterministic function $f_{\mathrm{B}}$ of $\left(Y_{\mathrm{In}}, \bar{T}\right) \cdot{ }^{19}$

Let $\mathcal{S}=\left\{(y, \bar{t}, z) \in \operatorname{Supp}\left(Y_{\mathrm{In}}, \bar{T}\right) \times \mathbb{R}:\left(f_{\mathrm{B}}(y, \bar{t})-z \bmod m\right) \in\{m-\Delta, \ldots, 0, \ldots, \Delta\}\right\}$. It follows that

$$
\begin{aligned}
& \operatorname{Pr}\left[\left|Y_{\text {out }}-\operatorname{IP}\left(X_{\text {In }}, Y_{\text {In }}\right)\right| \leq \Delta\right] \\
& \leq \operatorname{Pr}\left[\left(Y_{\text {In }}, \bar{T}, \operatorname{IP}\left(X_{\text {In }}, Y_{\text {In }}\right)\right) \in \mathcal{S}\right] \\
& =\operatorname{Pr}\left[\left(Y_{\text {In }}, \bar{T}, \operatorname{IP}\left(X_{\text {In }}, Y_{\text {In }}\right) \bmod m\right) \in \mathcal{S}\right] \\
& \leq \operatorname{Pr}\left[\left(\widehat{Y}_{\bar{T}}, \bar{T}, U_{m}\right) \in \mathcal{S}\right]+\operatorname{SD}\left(\left(Y_{\text {In }}, \bar{T}, \operatorname{IP}\left(X_{\text {In }}, Y_{\text {In }}\right) \bmod m\right),\left(\widehat{Y}_{\bar{T}}, \bar{T}, U_{m}\right)\right) \\
& \leq \operatorname{Pr}\left[\left(\widehat{Y}_{\bar{T}}, \bar{T}, U_{m}\right) \in \mathcal{S}\right]+\mathrm{E}\left[4 s \gamma_{\bar{T}}+\tau / 3\right] \\
& \leq 2 \Delta / m+16 s \gamma+\tau / 3 \\
& \leq \tau
\end{aligned}
$$

The second inequality holds by Equation (15), the third one since $\mathrm{E}\left[\gamma_{\bar{T}}\right] \leq 4 \gamma$, and the last one since $\frac{\Delta}{m}=\gamma / 6$ and since, by assumption, $\tau \geq 48 s \gamma$.

Proof of Claim 41. Let $X_{j}$ denote the $j$ 'th bit in $X_{\text {In }}$. For $i \in[s]$ and $(x, \bar{t}) \in \operatorname{Supp}\left(X_{\text {In }}, \bar{T}\right)$, define

$$
\begin{aligned}
\rho_{X}(i, x, \bar{t}) & :=\frac{\operatorname{Pr}\left[X_{i}=0 \mid X_{1}=x_{1}, \ldots X_{i-1}=x_{i-1}, X_{i+1}=x_{i+1}, \ldots, X_{s}=x_{s}, \bar{T}=\bar{t}\right]}{\operatorname{Pr}\left[X_{i}=1 \mid X_{1}=x_{1}, \ldots X_{i-1}=x_{i-1}, X_{i+1}=x_{i+1}, \ldots, X_{s}=x_{s}, \bar{T}=\bar{t}\right]} \\
& =\frac{\operatorname{Pr}\left[\bar{T}=\bar{t} \mid X_{1}=x_{1}, \ldots X_{i-1}=x_{i-1}, X_{i}=0, X_{i+1}=x_{i+1}, \ldots, X_{s}=x_{s}\right]}{\operatorname{Pr}\left[\bar{T}=\bar{t} \mid X_{1}=x_{1}, \ldots X_{i-1}=x_{i-1}, X_{i}=1, X_{i+1}=x_{i+1}, \ldots, X_{s}=x_{s}\right]},
\end{aligned}
$$

where the equality holds by the uniformity of $X_{\text {In }}$ (using Bayes' Rule), and let $\mathcal{S}_{X}=$ $\left\{(i, x, \bar{t}): \rho_{X}(i, x, \bar{t}) \notin\left[e^{-(1.1+\alpha)}, e^{(1.1+\alpha)}\right]\right\}$. Define $\mathcal{S}_{Y}$ analogously for $Y_{\text {In }}$. For $\bar{t} \in \operatorname{Supp}(\bar{T})$, set $\gamma_{\bar{t}}:=\max \left\{\operatorname{Pr}_{i \leftarrow[s], x \leftarrow X_{\text {In } \mid \bar{t}}}\left[(i, x, \bar{t}) \in \mathcal{S}_{X}\right], \operatorname{Pr}_{i \leftarrow[s], y \leftarrow Y_{\text {In } \mid \bar{t}}}\left[(i, y, \bar{t}) \in \mathcal{S}_{Y}\right]\right\}$. It follows that $X_{\text {In } \mid \bar{t}}$ and $Y_{\operatorname{In} \mid \bar{t}}$ are $\left(e^{-(1.1+\alpha)}, \gamma_{\bar{t}}\right)$-strongly-unpredictable bit sources, and hence, Corollary 32 yields that both $X_{\operatorname{In} \mid \bar{t}}$ and $Y_{\operatorname{In} \mid \bar{t}}$ are $2 s \gamma_{\hat{t}}$-close to some $\left(e^{-(1.1+\alpha)} / 3\right)$-unpredictable bit sources, yielding the first requirement of the claim.

For the second requirement of the claim, applying Lemma 28 with $\nu=1.1$ yields that

$$
\max \left\{\begin{aligned}
\left.\operatorname{Pr}_{(x, \bar{t}) \leftarrow\left(X_{\text {In }}, \bar{T}\right)}\left[(i, x, \bar{t}) \in \mathcal{S}_{X}\right], \underset{(y, \bar{t}) \leftarrow\left(Y_{\text {In }}, \bar{T}\right)}{\operatorname{Pr}}\left[(i, y, \bar{t}) \in \mathcal{S}_{Y}\right]\right\} & \leq \gamma \cdot \frac{1+e^{-(1.1+\alpha)}}{1-e^{-1.1}} \\
& <2 \gamma
\end{aligned}\right.
$$

\footnotetext{
${ }^{19}$ For an arbitrary function $f_{\mathrm{B}}$, consider its variant $f_{\mathrm{B}}^{\prime}$ that applies $f_{\mathrm{B}}$ on a random view that is consistent with $\left(Y_{\mathrm{In}}, \bar{T}\right)$. Clearly, $f_{\mathrm{B}}^{\prime}$ computes the inner product correctly with the same probability as $f_{\mathrm{B}}$ does, and its output is a randomized function of (only) $\left(Y_{\mathrm{In}}, \bar{T}\right)$. Finally, the deterministic function $f_{\mathrm{B}}^{\prime \prime}$ that applies $f_{\mathrm{B}}^{\prime}$ with the best choice of random coins, computes the inner product correctly no worse than $f_{\mathrm{B}}^{\prime}$ does, and thus no worse than $f_{\mathrm{B}}$.
} 
for every $i \in[s],{ }^{20}$ and we conclude that

$$
\begin{aligned}
\mathrm{E}\left[\gamma_{\bar{T}}\right] & \leq \underset{\bar{t} \leftarrow \bar{T}}{\mathrm{E}}\left[\operatorname{Pr}_{i \leftarrow[s], x \leftarrow X_{\text {In } \mid \bar{t}}}\left[(i, x, \bar{t}) \in \mathcal{S}_{X}\right]+\underset{i \leftarrow[s], y \leftarrow Y_{\text {In } \mid \bar{t}}}{\operatorname{Pr}}\left[(i, y, \bar{t}) \in \mathcal{S}_{Y}\right]\right] \\
& \leq \mathrm{E}\left[2 \cdot \max \left\{\underset{(x, \bar{t}) \leftarrow\left(X_{\text {In }}, \bar{T}\right)}{\operatorname{Pr}}\left[(i, x, \bar{t}) \in \mathcal{S}_{X}\right], \underset{(y, \bar{t}) \leftarrow\left(Y_{\text {In }}, \bar{T}\right)}{\operatorname{Pr}}\left[(i, y, \bar{t}) \in \mathcal{S}_{Y}\right]: i \in[s]\right\}\right] \\
& <4 \gamma .
\end{aligned}
$$

Proof of Claim 42. Let $\lambda_{1}=\lambda / \eta>0$, where $\lambda \in(0,1)$ is determined later, and note that $s=$ $\left(\frac{\Delta \cdot \log s}{\lambda_{1} \cdot \tau \cdot \eta}\right)^{2}$. Let $z_{1}=z_{1}(\lambda, \alpha)$ be such that $s \geq z_{1}$ implies $\log s \geq 1 / \lambda_{1}$. Note that

$$
\begin{aligned}
s & \geq \frac{1}{\lambda_{1}^{2}} \cdot\left(\frac{\Delta}{\tau \eta}\right)^{2} \cdot\left(\log \left(\frac{\Delta}{\tau \eta}\right)\right)^{2} \\
& =\frac{1}{\lambda_{1}^{2}} \cdot \frac{m^{2}}{36 \cdot \eta^{2}} \cdot\left(\log \left(\frac{m}{6 \eta}\right)\right)^{2} \\
& \geq \frac{1}{36 \cdot \lambda_{1}^{2}} \cdot \frac{m^{2}}{\eta \beta} \cdot\left(\log \left(\frac{m}{6 \eta}\right)\right)^{2}
\end{aligned}
$$

for every $s \geq z_{1}$, where the last inequality holds since, by inspection, $\beta \geq \eta$.

Let $z_{2}=z_{2}(\lambda, \alpha)$ be such that $s \geq z_{2}$ implies $\frac{m}{6 \cdot \eta} \geq 2$, and let $z=\max \left\{z_{1}, z_{2}\right\}$. Let $\kappa=$ $\kappa(\alpha, \nu)=1+\max \left\{\log \left(\frac{6 \eta}{\beta}\right), \log \left(\frac{6 \eta}{\nu / 3}\right)\right\}$ and let $\lambda=\lambda(\alpha, \nu) \in(0,1)$ be such that $\frac{1}{36 \cdot \lambda_{1}^{2}} \geq c \cdot \kappa^{2}$. Fix $s \geq z$. Since $\left(\frac{m}{6 \eta}\right)^{\kappa} \geq \max \left\{\frac{m}{\beta}, \frac{m}{\nu / 3}\right\}$, Equation (19) yields that

$$
\begin{aligned}
s & \geq c \cdot \kappa^{2} \cdot \frac{m^{2}}{\eta \beta} \cdot\left(\log \left(\frac{m}{6 \eta}\right)\right)^{2} \\
& \geq c \cdot \frac{m^{2}}{\eta \beta} \cdot \log \left(\frac{m}{\beta}\right) \cdot \log \left(\frac{m}{\tau / 3}\right),
\end{aligned}
$$

concluding the claim's proof.

Lower bound for oracle-aided, sampled-input protocols. We now use Theorem 12 to give a variant of Theorem 40 for (sampled-input) oracle-aided protocols. We start by showing that the existence of differentially private, oracle-aided, sampled-input protocols implies the existence of no-oracle, sampled-input protocols, incurring no loss in privacy, and a minor loss in accuracy.

Lemma 43. Let $\mathcal{F}$ be a function family, let $\pi$ be an oracle-aided, s-bit sampled-input protocol, and let $g:\{0,1\}^{S} \times\{0,1\}^{S} \mapsto \mathbb{R}$ be a deterministic function. Assume that the pair $(\mathcal{F}, \pi)$ has a $(T, \varepsilon)$ mapping, and assume that $\pi$ is a $(\beta, d)$-SI-approximation for $g$ with respect to $\mathcal{F}$ and party $\mathrm{P}$, and satisfies $(T, \alpha, \gamma)$-differential privacy with respect to $\mathcal{F}$. Then, the no-oracle, s-bit sampled-input

\footnotetext{
${ }^{20}$ We note that Lemma 28 is stated for differentially private mechanisms. Nevertheless, its proof for sampled-input protocols readily follows from the original proof.
} 
protocol $\widetilde{\pi}=(\widetilde{\mathrm{A}}, \widetilde{\mathrm{B}})$, guaranteed by the $(T, \varepsilon)$-mapping, is a $(\beta+\varepsilon, d)$-SI-approximation for $g$ with respect to party $\mathrm{P}$, and is $(\alpha, \gamma)$-differentially private.

Furthermore, the sampled input of party A (resp. B) and the sampled input of party $\widetilde{\mathrm{A}}$ (resp. $\widetilde{\mathrm{B}}$ ) are identically distributed.

Proof. Let $\tilde{\pi}=(\widetilde{\mathrm{A}}, \widetilde{\mathrm{B}})$ and Map be the no-input, no-oracle protocol and oracle-aided algorithm guaranteed by Definition 11 with respect to $\pi$ and $\mathcal{F}$. We first argue that $\tilde{\pi}$ satisfies $(\alpha, \gamma)$ differential privacy. Assume to the contrary that this is not the case. Specifically, assume without loss of generality that there exists a (no-oracle) adversary $\tilde{D}$, such that

$$
\operatorname{Pr}_{\tilde{v} \leftarrow\langle\widetilde{\pi}\rangle}\left[\tilde{\mathrm{D}}(\operatorname{trans}(\tilde{v}))=1 \mid \operatorname{SInp}^{\widetilde{\mathrm{A}}}(\tilde{v})=x\right]>e^{\alpha} \cdot \operatorname{Pr}_{\tilde{v} \leftarrow\langle\widetilde{\pi}\rangle}\left[\tilde{\mathrm{D}}(\operatorname{trans}(\tilde{v}))=1 \mid \operatorname{SInp}^{\widetilde{\mathrm{A}}}(\tilde{v})=x^{\prime}\right]+\gamma
$$

for some $x, x^{\prime} \in\{0,1\}^{s}$ with $H_{d}\left(x, x^{\prime}\right)=1$. Consider the adversary $\mathrm{D}$ for $\pi$ that on a given transcript $\bar{t}$ (of an execution of $\pi$ with access to $f$ ) applies $\tilde{\mathrm{D}}$ to $\operatorname{Map}^{f}(\bar{t})$. We claim that

$$
\begin{aligned}
\operatorname{Pr}_{f \leftarrow \mathcal{F}, v \leftarrow\left\langle\pi^{f}\right\rangle}\left[\mathrm{D}^{f}(\operatorname{trans}(v))=1 \mid \operatorname{SInp}^{\mathrm{A}}(v)=x\right] \\
>e^{\alpha} \cdot \operatorname{Pr}_{f \leftarrow \mathcal{F}, v \leftarrow\left\langle\pi^{f}\right\rangle}\left[\mathrm{D}^{f}(\operatorname{trans}(v))=1 \mid \operatorname{SInp}^{\mathrm{A}}(v)=x^{\prime}\right]+\gamma
\end{aligned}
$$

To see that Equation (21) holds, note that by the furthermore statement of the first item in Definition 11, the transcript together with the sampled input of $\widetilde{A}$ in a random execution of $\tilde{\pi}$ (i.e., $\left(\operatorname{trans}(\tilde{v}), \operatorname{SInp}^{\widetilde{A}}(\tilde{v})\right)$, where $\left.\tilde{v} \leftarrow\langle\tilde{\pi}\rangle\right)$, are (jointly) identically distributed as the value of Map applied to the transcript and the sampled input of $\mathrm{A}$ in a random execution of $\pi$ (i.e., $\left(\operatorname{Map}^{f}(\operatorname{trans}(v)), \operatorname{SInp}^{\mathrm{A}}(v)\right)$, where $v \leftarrow\left\langle\pi^{f}\right\rangle$ for $\left.f \leftarrow \mathcal{F}\right)$. In addition, by Definition 11, D makes at most $T$ oracle calls. Hence, we obtain a contradiction to the $(T, \alpha, \gamma)$-differential privacy of $\pi$, yielding that the protocol $\widetilde{\pi}$ must be $(\alpha, \gamma)$-differentially private.

We conclude the proof by showing that $\tilde{\pi}$ is a good approximation for $g$ with respect to any party $P \in\{\widetilde{A}, \widetilde{B}\}$. Specifically, we show that

$$
\operatorname{Pr}_{\tilde{v} \leftarrow\langle\tilde{\pi}\rangle}\left[\left|g\left(\operatorname{SInp}^{\widetilde{A}}(\tilde{v}), \operatorname{Sinp}^{\widetilde{B}}(\tilde{v})\right)-\operatorname{AOut}^{\mathrm{P}}(\tilde{v})\right| \geq d\right]<\beta+\varepsilon .
$$

By the first item in Definition 11, we have that the (actual) joint outputs of the parties in a random execution of $\pi$, are in statistical distance at most $\varepsilon$ from the (actual) joint outputs of the parties in a random execution of $\widetilde{\pi}$. Formally, if we let $\mathcal{D}_{\widetilde{\pi}}=\left(\operatorname{SInp}^{\widetilde{A}}(\tilde{v}), \operatorname{SInp}^{\widetilde{B}}(\tilde{v}), \operatorname{AOut}^{\mathrm{P}}(\tilde{v})\right)_{\tilde{v} \leftarrow\langle\tilde{\pi}\rangle}$ and $\mathcal{D}_{\pi}=\left(\operatorname{SInp}^{\mathrm{A}}(v), \operatorname{SInp}^{\mathrm{B}}(v), \operatorname{AOut}^{\mathrm{P}}(v)\right)_{f \leftarrow \mathcal{F}, v \leftarrow\left\langle\pi^{f}\right\rangle}$, then we have that $\operatorname{SD}\left(\mathcal{D}_{\tilde{\pi}}, \mathcal{D}_{\pi}\right) \leq \varepsilon$. Hence, Equation (22) follows from the accuracy of $\pi$, i.e., since we have that

$$
\underset{f \leftarrow \mathcal{F}, v \leftarrow\langle\pi f\rangle}{\operatorname{Pr}}\left[\left|g\left(\operatorname{SInp}^{\mathrm{A}}(v), \operatorname{SInp}^{\mathrm{B}}(v)\right)-\operatorname{AOut}^{\mathrm{P}}(v)\right| \geq d\right]<\beta
$$

To verify this, let $\mathcal{S}=\left\{(x, y, w) \in \operatorname{Supp} \mathcal{D}_{\tilde{\pi}}:|g(x, y)-w| \geq d\right\}$ and observe that the probability of falling into $S$ according to $\mathcal{D}_{\widetilde{\pi}}$ can be larger than the probability of falling into $S$ according to $\mathcal{D}_{\pi}$ (which is bounded by $\beta$ ), by at most the statistical distance between $\mathcal{D}_{\widetilde{\pi}}$ and $\mathcal{D}_{\pi}$.

The furthermore statement follows from the furthermore statement of the first item in Definition 11 . 
We now combine Lemma 43 and theorem 40 to prove a lower bound on the accuracy of oracleaided, sampled-input protocols that are differentially private.

Proposition 44. For numbers $\nu>0$ and $\alpha \geq 0$, there exist numbers $\lambda>0$ and $z \in \mathbb{N}$ such that the following holds. Let $\mathcal{F}$ be a function family and let $\pi=(\mathrm{A}, \mathrm{B})$ be an oracle-aided, s-bit sample-input protocol. For $f \in \mathcal{F}$, let $X_{\mathrm{In}}^{f}$ and $Y_{\mathrm{In}}^{f}$ be the sampled inputs of $\mathrm{A}$ and $\mathrm{B}$, respectively, and let $X_{\text {out }}^{f}$ and $Y_{\text {out }}^{f}$ be the actual outputs of $\mathrm{A}$ and $\mathrm{B}$, respectively, induced by a random execution of $\pi^{f}$.

Assume that both $X_{\mathrm{In}}^{f}$ and $Y_{\mathrm{In}}^{f}$ are uniformly distributed over $\{0,1\}^{s}$ for every $f \in \mathcal{F}$, that $\pi$ is $(T, \alpha, \gamma)$-differentially private with respect to $\mathcal{F}$, that the pair $(\mathcal{F}, \pi)$ has a $(T, \varepsilon)$-mapping, and finally that $s \geq z$, then for some $f \in \mathcal{F},{ }^{17}$ it holds that

$$
\operatorname{Pr}\left[\left|Y_{\text {out }}^{f}-\operatorname{IP}\left(X_{\text {In }}^{f}, Y_{\text {In }}^{f}\right)\right| \leq \Delta:=\lambda \cdot \frac{\sqrt{s}}{\log s} \cdot(\tau-\varepsilon)\right] \leq \tau
$$

for every $1 \geq \tau$ with $\tau-\varepsilon \geq \max \{48 s \gamma, \nu\} .{ }^{15}$ The same holds for $X_{\text {out }}^{f}$.

Proof. Given values for $\nu$ and $\alpha$, set $\lambda$ and $z$ to be as in Theorem 40 . Let $\mathcal{F}$ and $\pi$ be as in the statement of the proposition. Since $\pi$ is assumed to be $(T, \alpha, \gamma)$-differentially private with respect to $\mathcal{F}$, and since the pair $(\mathcal{F}, \pi)$ is assumed to have a $(T, \varepsilon)$-mapping, it follows from Lemma 43 that the no-oracle, sampled-input protocol $\widetilde{\pi}$ (guaranteed by this mapping) is $(\alpha, \gamma)$-differentially private.

Since $X_{\text {In }}$ and $Y_{\text {In }}$ are uniformly distributed over $\{0,1\}^{s}$, it follows from the furthermore statement of Lemma 43 that the same holds for the sampled inputs of both parties in $\tilde{\pi}$. Hence, Theorem 40 yields that for $s \geq z$ and $\tau$ such that $\tau^{\prime}:=\tau-\varepsilon \geq \max \{48 s \gamma, \nu\}$, it holds that $\tilde{\pi}$ is not a $\left(1-\tau^{\prime}, \Delta\right)$-SI-approximation for $\Delta=\lambda \cdot \frac{\sqrt{s}}{\log s} \cdot \tau^{\prime}$. By Lemma $43, \pi$ is not a $\left(1-\tau^{\prime}+\varepsilon, \Delta\right)$ SI-approximation, namely, $\operatorname{Pr}\left[\left|Y_{\text {out }}-\operatorname{IP}\left(X_{\text {In }}, Y_{\text {In }}\right)\right| \leq \Delta\right] \leq \tau^{\prime}-\varepsilon=\tau$.

\subsubsection{Limits on Uniform-Input Executions}

The focus of this section is on executions of differentially private protocols, where the inputs of the parties are chosen uniformly at random. Towards proving a lower-bound on the accuracy of approximating the inner-product function in such executions, we map a uniform-input execution of a with-input protocol to the sampled-input variant of this protocol (as defined in Definition 34). Roughly speaking, in the sampled-input variant of a protocol, the parties sample their inputs at random at the beginning of an execution.

We next define what it means for a protocol to approximate a function with good probability when the inputs of the parties are uniformly selected.

Definition 45 (good random-approximations). Let $g:\{0,1\}^{s} \times\{0,1\}^{s} \mapsto \mathbb{R}$ be a deterministic function, and let $\pi=(\mathrm{A}, \mathrm{B})$ be an oracle-aided, s-bit input protocol. Protocol $\pi$ is a $(\beta, d)$-randomapproximation for $g$ with respect to a function family $\mathcal{F}$ and $\mathrm{P} \in\{\mathrm{A}, \mathrm{B}\}$, if for every $f \in \mathcal{F}$, it holds that

$$
\underset{x, y \leftarrow\{0,1\}^{s}, v \leftarrow\left\langle\pi^{f}(x, y)\right\rangle}{\operatorname{Pr}}\left[\left|g(x, y)-\operatorname{out}^{\mathrm{P}}(v)\right|>d\right]<\beta .
$$

Protocol $\pi$ is a $(\beta, d)$-random-approximation for $g$ with respect to $\mathcal{F}$, if it is a $(\beta, d)$-randomapproximation for $g$ with respect to $\mathcal{F}$ and both parties. 
The following observation allows us to use the lower bound stated in Lemma 43, to derive a similar bound for with-input protocols, when the inputs of the parties are chosen uniformly at random.

Lemma 46. Let $g:\{0,1\}^{s} \times\{0,1\}^{s} \mapsto \mathbb{R}$ be a deterministic function and let $\mathcal{F}$ be some oracle family. Assume that there exists an oracle-aided, s-bit input protocol $\pi=(\mathrm{A}, \mathrm{B})$ that is a $(\beta, d)$ random-approximation for $g$ with respect to $\mathcal{F}$ and party $\mathrm{P}$, and is $(k, \alpha, \gamma)$-differential privacy with respect to $\mathcal{F}$. Then $\mu(\pi)$ is a $(\beta, d)$-SI-approximation for $g$ with respect to $\mathcal{F}$ and $\mathrm{P}$, and $(k, \alpha, \gamma)$-differentially private with respect to $\mathcal{F}$.

Proof. Immediate by definition.

Combining Proposition 44 and Lemma 46 yields the following result.

Proposition 47. For numbers $\nu>0$ and $\alpha \geq 0$, there exist numbers $\lambda>0$ and $z \in \mathbb{N}$ such that the following holds. Let $\mathcal{F}$ be a function family and let $\pi=(\mathrm{A}, \mathrm{B})$ be an oracle-aided, s-bit input protocol. For $f \in \mathcal{F}$, let $X_{\mathrm{In}}^{f}$ and $Y_{\mathrm{In}}^{f}$ be the inputs of $\mathrm{A}$ and $\mathrm{B}$, respectively, and let $X_{\text {out }}^{f}$ and $Y_{\text {out }}^{f}$ be the outputs of $\mathrm{A}$ and $\mathrm{B}$, respectively, induced by a random execution of $\pi^{f}$.

Assume that both $X_{\mathrm{In}}^{f}$ and $Y_{\mathrm{In}}^{f}$ are independently and uniformly chosen from $\{0,1\}^{\text {s }}$ for every $f \in \mathcal{F}$, that $\pi$ is $(T, \alpha, \gamma)$-differentially private with respect to $\mathcal{F}$, that the pair $(\mathcal{F}, \mu(\pi))$ has a $(T, \varepsilon)$-mapping (where $\mu(\pi)$ is sampled-input variant of $\pi$ ), and finally that $s \geq z$. Then, for some $f \in \mathcal{F},{ }^{17}$ it holds that

$$
\operatorname{Pr}\left[\left|Y_{\text {out }}^{f}-\operatorname{IP}\left(X_{\text {In }}^{f}, Y_{\text {In }}^{f}\right)\right| \leq \Delta:=\lambda \cdot \frac{\sqrt{s}}{\log s} \cdot(\tau-\varepsilon)\right] \leq \tau
$$

for every $1 \geq \tau$ with $\tau-\varepsilon \geq \max \{48 s \gamma, \nu\} .{ }^{15}$ The same holds for $X_{\text {out }}^{f}$.

Proof. Given values for $\nu$ and $\alpha$, set $\lambda$ and $z$ to be as in Proposition 44 . Let $\mathcal{F}$ and $\pi$ be as in the statement of the proposition. Let $\mu(\pi)$ be the (oracle-aided) sampled-input variant of $\pi$ (see Definition 34). By construction, the sampled inputs of both parties in $\mu(\pi)$ are uniformly distributed. Since $\pi$ is assumed to be $(T, \alpha, \gamma)$-differentially private with respect to $\mathcal{F}$, it follows by Lemma 46 that $\mu(\pi)$ is also $(T, \alpha, \gamma)$-differentially private with respect to $\mathcal{F}$. Since the pair $(\mathcal{F}, \mu(\pi))$ is assumed to have a $(T, \varepsilon)$-mapping, it follows from Proposition 44 that for $s \geq z$ and $\tau$ such that $\tau^{\prime}:=\tau-\varepsilon \geq \max \{48 s \gamma, \nu\}$, the protocol $\mu(\pi)$ is not a $\left(1-\tau^{\prime}+\varepsilon, \Delta\right)$-SI-approximation for $\Delta=\lambda \cdot \frac{\sqrt{s}}{\log s} \cdot \tau^{\prime}$. Hence, by Lemma $46, \pi$ is not a $\left(1-\tau^{\prime}+\varepsilon, \Delta\right)$-random-approximation, namely, $\operatorname{Pr}\left[\left|Y_{\text {out }}-\operatorname{IP}\left(X_{\text {In }}, Y_{\text {In }}\right)\right| \leq \Delta\right] \leq \tau^{\prime}-\varepsilon=\tau$.

\subsubsection{Limits on Arbitrary Protocols - Proving Theorem 35}

The results presented in the previous section yield the lower-bounds of Section 4.2.2 in a straightforward manner. That is, the lower-bound on the accuracy of differentially private protocols, with respect to executions where inputs are selected uniformly at random, easily implies a similar lower bound for arbitrary executions of such protocols. Intuitively, this is because if a protocol errs with probability $\beta$ on uniform inputs, then there must be a specific choice of inputs for the parties on which the protocol errs with probability at least $\beta$.

Proof of Theorem 35. Immediate, by taking $x$ and $y$ that maximize the probability in Equation (11), and using Proposition 47 to bound this probability from below. 


\subsection{Secure Function Evaluation}

In this section we apply our main result to show that when given access to a random member of a simple function family (e.g., the all-function family), no oracle-aided protocol can securely compute any no-input functionality that cannot be (almost) securely computed by a no-oracle protocol.

In semi-honest no-input secure function evaluation, two parties $\mathrm{A}$ and $\mathrm{B}$ wish to compute some (possibly randomized) functionality privately and correctly. Let $G=\left(G_{\mathrm{A}}, G_{\mathrm{B}}\right)$ be a distribution over $\mathcal{A} \times \mathcal{B}$, where $G_{\mathrm{A}}$ and $G_{\mathrm{B}}$ denote its marginal distributions over $\mathcal{A}$ and $\mathcal{B}$ respectively. The parties wish to perform a computation, where party A learns $g_{\mathcal{A}}$ and party B learns $g_{\mathcal{B}}$ for $g=$ $\left(g_{\mathcal{A}}, g_{\mathcal{B}}\right) \leftarrow G$, but nothing else. Since the parties are semi-honest, they will always follow the prescribed protocol. A corrupted party, however, may try to use its view in the computation to infer additional information after the computation terminates.

\subsubsection{Standard Definitions}

Definition 48 (no-input secure function evaluation). Let $\mathcal{A}$ and $\mathcal{B}$ be sets, and let $G=\left(G_{\mathrm{A}}, G_{\mathrm{B}}\right)$ be a distribution over $\mathcal{A} \times \mathcal{B}$, where $G_{\mathrm{A}}$ and $G_{\mathrm{B}}$ denote its marginal distributions over $\mathcal{A}$ and $\mathcal{B}$ respectively. A two-party, oracle-aided protocol $\pi=(\mathrm{A}, \mathrm{B})$ is an $(m, k, \delta)$-secure protocol for $G$ with respect to a function family $\mathcal{F}$, for $\delta \in[0,1]$ and $m, k \in \mathbb{N}$, if the following conditions hold:

Correctness: $\pi$ is a $\delta$-correct implementation of $G$ with respect to $\mathcal{F}$. That is, for every $f \in \mathcal{F}$,

$$
\mathrm{SD}\left(\left(\operatorname{out}^{\mathrm{A}}(v), \operatorname{out}^{\mathrm{B}}(v)\right)_{v \leftarrow\left\langle\pi^{f}\right\rangle}, G\right) \leq \delta .
$$

Privacy: $\pi$ is an $(m, k, \delta)$-private implementation of $G$ with respect to $\mathcal{F}:$ for every $P \in\{\mathrm{A}, \mathrm{B}\}$ there exists an m-query algorithm (simulator) Simp such that for any $k$-query distinguisher $\mathrm{D}$,

$$
\underset{f \leftarrow \mathcal{F}}{\mathrm{E}}\left[\mid \operatorname{Pr}\left[\mathrm{D}^{f}\left(\left(\operatorname{Sim}_{\mathrm{P}}^{f}\left(g_{\mathrm{P}}\right), g_{\mathrm{P}}\right)_{g_{\mathrm{P}} \leftarrow G_{\mathrm{P}}}\right)=1\right]-\operatorname{Pr}\left[\mathrm{D}^{f}\left(\left(v_{\mathrm{P}}, \text { out }^{\mathrm{P}}(v)\right)_{v \leftarrow\left\langle\pi^{f}\right\rangle}\right)=1\right] \mid\right] \leq \delta .
$$

A protocol $\pi$ is a $\delta$-secure (no-oracle) implementation of $G$ if it is a $(\cdot, \cdot, \delta)$-secure implementation of $G$ with respect to the trivial function family (i.e., the singleton family, whose only member returns $\perp$ on any query). A distribution $G$ is $\delta$-trivial, if $G$ has a $\delta$-secure no-oracle implementation.

\subsubsection{Limits on Oracle-Aided Secure Function Evaluation}

In the language of the above definitions, the main result of this section is stated as follows.

Theorem 49. Let $\mathcal{F}$ be a function family, and let $\pi$ be an oracle-aided protocol that is a $(\cdot, T, \delta)$ secure oracle-aided implementation of a distribution $G$ with respect to $\mathcal{F}$. Assume that the pair $(\mathcal{F}, \pi)$ has a $(T, \delta)$-mapping. Then, $G$ is $2 \delta$-trivial.

Proof. Let $G=\left(G_{\mathrm{A}}, G_{\mathrm{B}}\right)$ be a distribution over $\mathcal{A} \times \mathcal{B}$, where $G_{\mathrm{A}}$ and $G_{\mathrm{B}}$ denote its marginal distributions over $\mathcal{A}$ and $\mathcal{B}$ respectively, and let $\pi=(\mathrm{A}, \mathrm{B})$ be an oracle-aided implementation of $G$ that is $(m, T, \delta)$-secure ( $m$ will play no role in the proof) with respect to a function family $\mathcal{F}$. Assume that the pair $(\mathcal{F}, \pi)$ has a $(T, \delta)$-mapping. Let $\widetilde{\pi}=(\widetilde{\mathrm{A}}, \widetilde{\mathrm{B}})$ and Map be the no-input 
no-oracle protocol and oracle-aided algorithm, guaranteed by the assumption of the theorem. We next prove that $\widetilde{\pi}$ is a $2 \delta$-secure implementation of $G$.

The correctness property stems from the first item in Definition 11, yielding that

$$
\operatorname{SD}\left(\left(\operatorname{out}^{\widetilde{\mathrm{A}}}(v), \text { out }^{\widetilde{\mathrm{B}}}(v)\right)_{v \leftarrow\langle\widetilde{\pi}\rangle},\left(\operatorname{out}^{\mathrm{A}}(v), \text { out }^{\mathrm{B}}(v)\right)_{f \leftarrow \mathcal{F}, v \leftarrow\left\langle\pi^{f}\right\rangle}\right) \leq \delta .
$$

Hence, the $\delta$-correctness of $\pi$ yields that

$$
\operatorname{SD}\left(\left(\operatorname{out}^{\widetilde{A}}(v), \operatorname{out}^{\widetilde{B}}(v)\right)_{v \leftarrow\langle\widetilde{\pi}\rangle}, G\right) \leq \delta+\delta=2 \delta .
$$

For the privacy property, we construct a simulator $\widetilde{\operatorname{Sim}}_{\widetilde{P}}$ for party $\widetilde{P} \in\{\widetilde{A}, \widetilde{B}\}$. Let $\operatorname{Sim}_{P}$ be the oracle-aided simulator guaranteed to exist for $\pi$ and $\mathrm{A}$, and let Map be the $(T, \delta)$-mapping mapping guaranteed for $(\mathcal{F}, \pi)$. We define $\widetilde{\operatorname{Sim}}_{\widetilde{\mathrm{P}}}$ as follows:

Algorithm $50\left(\widetilde{\operatorname{Sim}_{\widetilde{\mathrm{P}}}}\right)$.

Input: $g_{\mathrm{P}} \in \operatorname{Supp}\left(G_{\mathrm{P}}\right)$

Operation:

1. Let $f \leftarrow \mathcal{F}$.

2. Let $v_{\mathrm{P}}=\operatorname{Sim}_{\mathrm{P}}^{f}\left(g_{\mathrm{P}}\right)$ and let $\bar{t}=\operatorname{trans}\left(v_{\mathrm{P}}\right)$.

3. Output a random view for $\widetilde{\mathrm{P}}$ in a random execution of $\widetilde{\pi}$, conditioned on $g_{\mathrm{P}}$ being $\widetilde{\mathrm{P}}$ 's output and $\tilde{t}=\operatorname{Map}^{f}(\bar{t})$ being the common transcript.

The success probability of the "best" distinguisher in the no-oracle model is defined by the statistical distance between the global output of a real execution and the global output of a simulation. Hence, it suffices to show that $\mathrm{SD}\left(\left(\widetilde{\operatorname{Sim}_{\widetilde{\mathrm{P}}}}\left(g_{\mathrm{P}}\right), g_{\mathrm{P}}\right)_{g_{\mathrm{P}} \leftarrow G_{\mathrm{P}}},\left(v_{\widetilde{\mathrm{P}}}, \text { out }^{\widetilde{\mathrm{P}}}(v)\right)_{v \leftarrow\langle\widetilde{\pi}\rangle}\right) \leq \delta$. By the definition of Simp and the triangle inequality, it follows that

$$
\mid \operatorname{Pr}\left[\mathrm{D}^{f}\left(\left(\operatorname{Sim}_{\mathrm{P}}^{f}\left(g_{\mathrm{P}}\right), g_{\mathrm{P}}\right)_{f \leftarrow \mathcal{F}, g_{\mathrm{P}} \leftarrow G_{\mathrm{P}}}\right)=1\right]-\operatorname{Pr}\left[\mathrm{D}^{f}\left(\left(v_{\mathrm{P}}, \text { out }^{\mathrm{P}}(v)\right)_{f \leftarrow \mathcal{F}, v \leftarrow\left\langle\pi^{f}\right\rangle}\right)\right] \mid \leq \delta
$$

for any $k$-query distinguisher D. Since Map is a $k$-query algorithm, Equation (27) yields that

$$
\operatorname{SD}\left(\left(\operatorname{Map}^{f}\left(\operatorname{trans}\left(\operatorname{Sim}_{\mathrm{P}}^{f}\left(g_{\mathrm{P}}\right)\right)\right), g_{\mathrm{P}}\right)_{f \leftarrow \mathcal{F}, g_{\mathrm{P}} \leftarrow G_{\mathrm{P}}},\left(\operatorname{Map}^{f}\left(\operatorname{trans}\left(v_{\mathrm{P}}\right)\right), \operatorname{out}^{\mathrm{P}}(v)\right)_{f \leftarrow \mathcal{F}, v \leftarrow\left\langle\pi^{f}\right\rangle}\right) \leq \delta .
$$

The first item in Definition 11 yields that $\left(\operatorname{Map}^{f}\left(\operatorname{trans}\left(v_{\mathrm{P}}\right)\right) \text {, } \operatorname{out}^{\mathrm{P}}(v)\right)_{f \leftarrow \mathcal{F}, v \leftarrow\left\langle\pi^{f}\right\rangle}$ is distributed identically to $\left(\operatorname{trans}(v) \text {, out } \widetilde{\mathrm{P}}^{\widetilde{1}}(v)\right)_{v \leftarrow\langle\widetilde{\pi}\rangle}$, and we conclude that

$$
\begin{aligned}
& \operatorname{SD}\left(\left(\widetilde{\operatorname{Sim}}_{\widetilde{\mathrm{P}}}\left(g_{\mathrm{P}}\right), g_{\mathrm{P}}\right)_{g_{\mathrm{P}} \leftarrow G_{\mathrm{P}}},\left(v_{\widetilde{\mathrm{P}}}, \operatorname{out}^{\widetilde{\mathrm{P}}}(v)\right)_{v \leftarrow\langle\widetilde{\pi}\rangle}\right) \\
& \leq \operatorname{SD}\left(\left(\operatorname{Map}^{f}\left(\operatorname{trans}\left(\operatorname{Sim}_{\mathrm{P}}^{f}\left(g_{\mathrm{P}}\right)\right)\right), g_{\mathrm{P}}\right)_{f \leftarrow \mathcal{F}, g_{\mathrm{P}} \leftarrow G_{\mathrm{P}}},\left(\operatorname{Map}^{f}\left(\operatorname{trans}\left(v_{\mathrm{P}}\right)\right), \operatorname{out}^{\mathrm{P}}(v)\right)_{f \leftarrow \mathcal{F}, v \leftarrow\left\langle\pi^{f}\right\rangle}\right) \\
& \leq \delta .
\end{aligned}
$$


Combining Theorems 12 and 49 yields the following result.

Theorem 51. Let $\mathcal{F}$ be a simple function family. For parameters $k, \ell \in \mathbb{N}$ and $\delta \in[0,1]$ with $k \geq 256 \cdot\left(\frac{\ell}{\delta}\right)^{2}$, and for a distribution $G$ that is not $2 \delta$-trivial, there exists no $\ell$-query oracle-aided protocol that is a $(\cdot, k, \delta)$-secure oracle-aided implementation of $G$ with respect to $\mathcal{F}$.

Proof. Let $\mathcal{F}$ be a simple function family and let $\pi$ be an $\ell$-query oracle-aided protocol. Let $G$ be a distribution that is not $2 \delta$-trivial. Theorem 12 yields that $(\mathcal{F}, \pi)$ has a $(T, \delta)$-mapping for

$T=256 \cdot\left(\frac{\ell}{\delta}\right)^{2}$. Hence, Theorem 49 yields that $\pi$ is not a $(\cdot, T, \delta)$-secure oracle-aided implementation of $G$ with respect to $\mathcal{F}$.

\subsection{Applications to Random Functions, and Black-Box Reductions to One-way Functions}

In this section we show that the all-function family is simple. and therefore Theorem 12 holds with respect to this family. We then use this fact to give limits on black-box reductions to one-way functions.

\subsubsection{Standard Definitions and Known Facts}

One-way functions and the all-function family. An efficiently computable function is oneway, if it is hard to invert on a random output.

Definition 52 (one-way functions). A polynomially-time computable function $f:\{0,1\}^{*} \mapsto\{0,1\}^{*}$ is one-way, if

$$
\operatorname{Pr}_{x \leftarrow\{0,1\}^{n}}\left[\mathrm{~A}\left(1^{n}, f(x)\right) \in f^{-1}(f(x))\right]=\operatorname{neg}(n)
$$

for any PРТM A.

We define the all-function family over a given input length, as the set of all length-preserving functions over this input length.

Definition 53 (the all-function family). For $n \in \mathbb{N}$, let $\mathcal{F}_{\mathrm{AF} n}$ be the family of all functions from $n$-bit strings to $n$-bit strings.

It is well known (cf., $[13,9]$ ) that random members of the all-function family are "one-way". Specifically, we use the following fact.

Fact 54. For any $\left(2^{n / 3}-1\right)$-query oracle-aided algorithm $\mathrm{A}$, it holds that

$$
\operatorname{Pr}_{f \leftarrow \mathcal{F}_{\mathrm{AF} n}}\left[\operatorname{Pr}_{x \leftarrow\{0,1\}^{n}}\left[\mathrm{~A}^{f}(f(x)) \in f^{-1}(f(x))\right]>2^{-n / 3}\right] \leq 2^{-n / 3} .
$$

Proof. It is easy to verify that

$$
\operatorname{Pr}_{f \leftarrow \mathcal{F}_{\mathrm{AF} n} ; ; \leftarrow \leftarrow\{0,1\}^{n}}\left[\mathrm{~A}^{f}(f(x)) \in f^{-1}(f(x))\right] \leq 2^{n / 3} / 2^{-n}=2^{-2 n / 3},
$$

and the proof follows by a straightforward averaging argument. 
Black-box reductions. Loosely speaking, a fully black-box reduction from a primitive $Q$ (e.g., key agreement protocol) to a primitive $P$ (e.g., one-way function ) is: (1) a construction of $Q$ out of $P$ that "ignores" the structure of the implementation of $P$ (i.e., uses it as a "black box"), and (2) a generic reduction from the security of $P$ to that of $Q$. In more details, such a reduction consists of a PPTM pair $(\mathrm{Q}, \mathrm{R})$ such that the following holds. (1) for every correct implementation $\mathrm{P}$ of $P$, it holds that $\mathrm{Q}^{\mathrm{P}}$ is a correct implementation of $Q$, and (2) for every adversary $\mathrm{A}$ that breaks (the security of $) Q^{P}$, it holds that $R^{P, A}$ breaks $P$. See [21] for a more formal discussion.

Cryptographic primitives are typically parameterized by the so called security parameter, which determines their security and functionality (e.g., the key length of the key-agreement protocol). For such primitives we consider a restricted form of black-box reductions that requires the reduction, and in particular the security proof $\mathrm{R}$, to work for every choice of the security parameter $n$, e.g., an algorithm that guesses the agreed key of the key-agrement protocol "too well" on security parameter $n$, can be used by the reduction to invert the one-way function on inputs of length $n$. See Definitions 57 and 59 for concrete examples. ${ }^{21}$

\subsubsection{The All-Function Family is Simple}

Lemma 55. For every $n \in \mathbb{N}$, the family $\mathcal{F}_{\mathrm{AF} n}$ is simple.

In the following we fix $n$ and let $\mathcal{F}_{\mathrm{AF}}=\mathcal{F}_{\mathrm{AF} n}$. Lemma 55 is proved via the following claim:

Claim 56. Let $\pi=(\mathrm{A}, \mathrm{B})$ be a no-input, oracle-aided protocol, let $\mathcal{I} \in\{0,1\}^{n} \times\{0,1\}^{n}$ be a set query/answer pairs, let $\left(v_{\mathrm{A}}, \cdot\right),\left(\cdot, v_{\mathrm{B}}\right) \in \operatorname{Supp}\left(\mathcal{V} \mathcal{I E W}^{\mathcal{F}_{\mathrm{AF}}, \pi}(\cdot, \mathcal{I})\right)$ and let $\mathcal{I}_{\mathrm{A}}\left[\right.$ resp., $\left.\mathcal{I}_{\mathrm{B}}\right]$ be the set of query/answer pairs that $\mathrm{A}$ makes in $v_{\mathrm{A}}$ [resp., B makes in $\left.v_{\mathrm{B}}\right]$. The following hold for every $(q, a) \in \mathcal{I}_{\mathrm{A}}$ (an analogous observation holds for $\left.(q, a) \in \mathcal{I}_{\mathrm{B}}\right)$ :

1. If $\left(v_{\mathrm{A}}, v_{\mathrm{B}}\right)$ is consistent (i.e., $\left.\operatorname{Pr}_{\Omega}\left[v_{\mathrm{A}}, v_{\mathrm{B}}\right]>0\right)$, then

$$
\underset{f \leftarrow\left(\mathcal{F}_{\mathrm{AF}} \mid \mathcal{I}_{\mathrm{A}}^{q} \cup \mathcal{I}_{\mathrm{B}}^{q} \cup \mathcal{I}\right)}{\operatorname{Pr}}[f(q)=a] \geq \underset{f \leftarrow\left(\mathcal{F}_{\mathrm{AF}} \mid \mathcal{I}_{\mathrm{A}}^{q} \cup \mathcal{I}\right)}{\operatorname{Pr}}[f(q)=a],
$$

where $\mathcal{I}_{\mathrm{A}}^{q}$ [resp., $\left.\mathcal{I}_{\mathrm{B}}^{q}\right]$ is the set of queries/answers asked in $v_{\mathrm{A}}$ [resp., $v_{\mathrm{B}}$ ] before $q$ is asked in $v_{\mathrm{A}}$.

2. If $\operatorname{Intersect}\left(v_{\mathrm{A}}, v_{\mathrm{B}}\right)=0$, then

$$
\operatorname{Pr}_{f \leftarrow\left(\mathcal{F}_{\mathrm{AF}} \mid \mathcal{I}_{\mathrm{A}}^{q} \cup \mathcal{I}_{\mathrm{B}}^{q} \cup \mathcal{I}\right)}[f(q)=a]=\underset{f \leftarrow\left(\mathcal{F}_{\mathrm{AF}} \mid \mathcal{I}_{\mathrm{A}}^{q} \cup \mathcal{I}\right)}{\operatorname{Pr}}[f(q)=a] .
$$

Proof. The proof of Item 2 is immediate. For proving Item 1 , assume first that $(q, \cdot) \in \mathcal{I}_{\mathrm{B}}^{q}$. Since the views are consistent, it follows that $\operatorname{Pr}_{f \leftarrow\left(\mathcal{F}_{\mathrm{AF}} \mid \mathcal{I}_{\mathrm{A}}^{q} \cup \mathcal{I}_{\mathrm{B}}^{q} \cup \mathcal{I}\right)}[f(q)=a]=1$ and the claim follows for this case. For the other case $\left((q, \cdot) \notin \mathcal{I}_{\mathrm{B}}^{q}\right)$, it is easy to verify that under this assumption $\operatorname{Pr}_{f \leftarrow\left(\mathcal{F}_{\mathrm{AF}} \mid \mathcal{I}_{\mathrm{A}}^{q} \cup \mathcal{I}_{\mathrm{B}}^{q} \cup \mathcal{I}\right)}[f(q)=a]=\operatorname{Pr}_{f \leftarrow\left(\mathcal{F}_{\mathrm{AF}} \mid \mathcal{I}_{\mathrm{A}}^{q} \cup \mathcal{I}\right)}[f(q)=a]$, concluding the claim's proof.

\footnotetext{
${ }^{21}$ We choose to focus on this simpler form of black-box reductions as it simplifies the proofs given in Sections 4.4 .3 and 4.4.4, and still seems to capture the same set of known reductions captured by the standard notion of black-box reductions.
} 
Proof of Lemma 55. Claim 56(2) immediately yields that $\operatorname{Intersect}_{\mathcal{I}}\left(v_{\mathrm{A}}, v_{\mathrm{B}}\right)=0$ implies $\operatorname{Dependent}_{\mathcal{I}}\left(v_{\mathrm{A}}, v_{\mathrm{B}}\right)=0$. For the other $\operatorname{direction}\left(\operatorname{Intersect} \mathcal{I}_{\mathcal{I}}\left(v_{\mathrm{A}}, v_{\mathrm{B}}\right)=1\right.$ implies $\operatorname{Dependent}_{\mathcal{I}}\left(v_{\mathrm{A}}, v_{\mathrm{B}}\right)=1$ ), we assume $\operatorname{Intersect}\left(v_{\mathrm{A}}, v_{\mathrm{B}}\right)=1$ and show that either $\alpha_{v_{\mathrm{A}} \mid v_{\mathrm{B}}}^{\mathcal{I}} \neq \alpha_{v_{\mathrm{A}}}^{\mathcal{I}}$ or $\alpha_{v_{\mathrm{B}} \mid v_{\mathrm{A}}}^{\mathcal{I}} \neq \alpha_{v_{\mathrm{B}}}^{\mathcal{I}}$.

We distinguish between two cases: assuming that $\left(v_{\mathrm{A}}, v_{\mathrm{B}}\right)$ are not consistent, it follows that $\operatorname{Pr}_{f \leftarrow \mathcal{F}_{\mathrm{AF}}}\left[\mathcal{I}, \mathcal{I}_{\mathrm{A}}, \mathcal{I}_{\mathrm{B}}\right]=0$ and therefore $\alpha_{v_{\mathrm{A}} \mid v_{\mathrm{B}}}^{\mathcal{I}}=0$. But since $v_{\mathrm{A}}$ is consistent with $\mathcal{I}$, it holds that $\alpha_{v_{\mathrm{A}}}^{\mathcal{I}} \neq 0$, yielding that Dependent ${ }_{\mathcal{I}}\left(v_{\mathrm{A}}, v_{\mathrm{B}}\right)=1$.

We complete the proof by considering the case that $\left(v_{\mathrm{A}}, v_{\mathrm{B}}\right)$ are consistent. Let $(q, a) \in\left(\mathcal{I}_{\mathrm{A}} \cap\right.$ $\left.\mathcal{I}_{\mathrm{B}}\right) \backslash \mathcal{I}$ be a query/answer pair whose existence guaranteed by the intersection assumption, and assume without loss of generality that $q$ was asked first by B. It follows that

1. $\operatorname{Pr}_{f \leftarrow\left(\mathcal{F}_{\mathrm{AF}} \mid \mathcal{I}_{\mathrm{A}}^{q} \cup \mathcal{I}_{\mathrm{B}}^{q} \cup \mathcal{I}\right)}[f(q)=a]=1$, and

2. $\operatorname{Pr}_{f \leftarrow\left(\mathcal{F}_{\mathrm{AF}} \mid \mathcal{I}_{\mathrm{A}}^{q} \cup \mathcal{I}\right)}[f(q)=a]=2^{-n}$.

Applying Claim 56(1) yields that $\alpha_{v_{\mathrm{A}} \mid v_{\mathrm{B}}}^{\mathcal{I}} \geq 2^{n} \cdot \alpha_{v_{\mathrm{A}}}^{\mathcal{I}}$, and therefore Dependent $\operatorname{I}_{\mathcal{I}}\left(v_{\mathrm{A}}, v_{\mathrm{B}}\right)=1$.

\subsubsection{Key Agreement Protocols}

Following Definition 20 and the discussion in Section 4.4.1, we define fully black-box reduction from key-agreement protocols to one-way functions as follows.

Definition 57 (fully black-box reduction from key agreement to one-way functions). $A$ PPTM triplet $(\mathrm{A}, \mathrm{B}, \mathrm{R})$ is a fully black-box reduction from an $(\alpha, \gamma)$-key-agreement protocol to one-way functions, where $\alpha$ and $\gamma$ are functions over $\mathbb{N}$, if the following holds for every $n \in \mathbb{N}$.

1. $(\mathrm{A}, \mathrm{B})$ is $(1-\alpha(n))$-consistent with respect to $\mathcal{F}_{\mathrm{AF} n}$ according to Definition 20.

2. For every function $f$ over $\{0,1\}^{n}$, for every algorithm $\mathrm{D}$ and $\delta>0$ such that $\operatorname{Pr}_{v \leftarrow\left\langle\left(\mathrm{A}^{f}, \mathrm{~B}^{f}\right)\left(1^{n}\right)\right\rangle}\left[\mathrm{D}(\operatorname{trans}(v))=\right.$ out $\left.^{P}(v)\right] \geq \gamma+\delta$ for some $P \in\{\mathrm{A}, \mathrm{B}\}$, algorithm $\mathrm{R}^{\mathrm{D}, f}$ inverts $f$ with probability at least $p(\delta)$, where $p \in$ poly is universal.

Combining Theorem 23, Fact 54, and Lemma 55, yields the following result.

Corollary 58. There exists no fully black-box reduction from an $(\alpha, \gamma)$-key-agreement protocol to one-way functions, with $1-\alpha(n)-\gamma(n)>1 / \operatorname{poly}(n)$.

Proof's sketch. Assume that there exists a fully black-box reduction (A, B, R) from an $(\alpha, \gamma)$-keyagreement protocol to one-way functions, with $1-\alpha(n)-\gamma(n)>1 / \operatorname{poly}(n)$. By Theorem 23 and Lemma 55, and a simple averaging argument, there exists a poly $(n)$-query algorithm $\mathrm{D}$ such that

$$
\left.\underset{f \leftarrow \mathcal{F}_{\mathrm{AF} n}}{\operatorname{Pr}} \underset{v \leftarrow\left\langle\left(\mathrm{A}^{f}, \mathrm{~B}^{f}\right)\left(1^{n}\right)\right\rangle}{\operatorname{Pr}}\left[\mathrm{D}^{f}(\operatorname{trans}(v))=\operatorname{out}^{P}(v)\right] \geq \gamma(n)+1 / \operatorname{poly}(n)\right] \geq 1 / \operatorname{poly}(n)
$$

It follows that $\operatorname{Pr}_{f \leftarrow \mathcal{F}_{\mathrm{AF} n}}\left[\operatorname{Pr}_{x \leftarrow\{0,1\}^{n}}\left[\mathrm{R}^{\mathrm{D}^{f}, f}(f(x)) \in f^{-1}(f(x))\right]>1 / \operatorname{poly}(n)\right]>1 / \operatorname{poly}(n)$, in contradiction to Fact 54. 


\subsubsection{Differentially Private Two-Party Computation}

Following Definitions 25 and 26 and the discussion in Section 4.4.1, we define fully black-box reduction from differentially private protocols to one-way functions as follows.

Definition 59 (fully black-box reduction from differentially private protocols to one-way functions). A PPTM triplet $(\mathrm{A}, \mathrm{B}, \mathrm{R})$ is a fully black-box reduction from an $(s, \beta, d, \alpha, \gamma)$-differentially private protocol for a function $g$ to one-way functions, where $s, \beta, d, \alpha$ and $\gamma$ are functions over $\mathbb{N}$, if the following holds for every $n \in \mathbb{N}$.

1. (A, B) is a $(\beta(n), d(n))$-approximation for $g$ with respect to $\mathcal{F}_{\mathrm{AF} n}$ according to Definition 26 .

2. For every function $f$ over $\{0,1\}^{n}$, for every algorithm $\mathrm{D}$ and $\delta>0$ such that

$$
\underset{v \leftarrow\left\langle\left(\mathrm{A}^{f}(x), \mathrm{B}^{f}(y)\right)\right\rangle}{\operatorname{Pr}}[\mathrm{D}(\operatorname{trans}(v))=1] \geq e^{\alpha(n)} \cdot \underset{v \leftarrow\left\langle\left(\mathrm{A}^{f}\left(x^{\prime}\right), \mathrm{B}^{f}(y)\right)\right\rangle}{\operatorname{Pr}}[\mathrm{D}(\operatorname{trans}(v))=1]+\gamma(n)+\delta
$$

for some $x, x^{\prime}, y \in\{0,1\}^{s(n)}$ with $H_{d}\left(x, x^{\prime}\right)=1$, or the analogue condition holds for some $y, y^{\prime}, x \in\{0,1\}^{s(n)}$ with $H_{d}\left(y, y^{\prime}\right)=1$, algorithm $\mathrm{R}^{\mathrm{D}, f}$ inverts $f$ with probability at least $p(\delta)$, where $p \in$ poly is universal.

Combining Theorem 36, Fact 54, and Lemma 55 yields the following result.

Corollary 60. For constants $\nu \in(0,1)$ and $\eta \geq 0$, there exist $\lambda>0$ such that the following holds. There exists no fully black-box reduction from an $(s, \beta, d, \alpha, \gamma)$-differentially private protocol for inner product to one-way functions, with $\alpha(n) \leq \eta, \gamma(n) \leq \frac{\nu}{48 \cdot s(n)}-1 / \operatorname{poly}(n), \beta(n) \leq \frac{1-\nu}{2}$ and $d(n) \leq \lambda \cdot \nu \cdot \frac{\sqrt{s(n)}}{\log s(n)}$ for infinitely many $n$ 's.

\section{References}

[1] B. Barak and M. Mahmoody. Merkle puzzles are optimal - an $O\left(n^{2}\right)$-query attack on any key exchange from a random oracle. In Advances in Cryptology - CRYPTO '09, pages 374-390, 2009 .

[2] A. Beimel, K. Nissim, and E. Omri. Distributed private data analysis: On simultaneously solving how and what. CoRR, abs/1103.2626, 2011.

[3] R. Canetti, O. Goldreich, and S. Halevi. On the random-oracle methodology as applied to length-restricted signature schemes. In Theory of Cryptography, First Theory of Cryptography Conference, TCC 2004, 2004.

[4] Y.-C. Chang, C.-Y. Hsiao, and C.-J. Lu. On the impossibilities of basing one-way permutations on central cryptographic primitives. In Advances in Cryptology - CRYPTO '02, pages 110-124, 2002 .

[5] D. Dachman-Soled, Y. Lindell, M. Mahmoody, and T. Malkin. On the black-box complexity of optimally-fair coin tossing. In tcc11, pages 450-467, 2011. 
[6] C. Dwork, F. McSherry, K. Nissim, and A. Smith. Calibrating noise to sensitivity in private data analysis. In Theory of Cryptography, Third Theory of Cryptography Conference, TCC 2006, pages 265-284, 2006.

[7] A. Fiat and A. Shamir. How to prove yourself: practical solutions to identification and signature problems. In Advances in Cryptology - CRYPTO '86, pages 186-194, 1987.

[8] R. Gennaro and L. Trevisan. Lower bounds on the efficiency of generic cryptographic constructions. In Proceedings of the 41st Annual Symposium on Foundations of Computer Science, pages 305-313, 2000.

[9] R. Gennaro, Y. Gertner, J. Katz, and L. Trevisan. Bounds on the efficiency of generic cryptographic constructions. SIAM Journal on Computing, 35(1):217-246, 2005.

[10] Y. Gertner, S. Kannan, T. Malkin, O. Reingold, and M. Viswanathan. The relationship between public key encryption and oblivious transfer. In Proceedings of the 32nd Annual ACM Symposium on Theory of Computing (STOC), 2000.

[11] S. Goldwasser and Y. Tauman-Kalai. On the (in)security of the fiat-shamir paradigm. In Proceedings of the 44th Annual Symposium on Foundations of Computer Science (FOCS), 2003.

[12] I. Haitner, J. J. Hoch, O. Reingold, and G. Segev. Finding collisions in interactive protocols - A tight lower bound on the round complexity of statistically-hiding commitments. In Proceedings of the 48th Annual Symposium on Foundations of Computer Science (FOCS), 2007.

[13] R. Impagliazzo and S. Rudich. Limits on the provable consequences of one-way permutations. In Proceedings of the 21st Annual ACM Symposium on Theory of Computing (STOC), pages 44-61. ACM Press, 1989.

[14] J. Kahn, M. Saks, and C. Smyth. A dual version of reimer's inequality and a proof of rudich's conjecture. In Computational Complexity, 2000. Proceedings. 15th Annual IEEE Conference on, pages $98-103,2000$.

[15] J. H. Kim, D. Simon, and P. Tetali. Limits on the efficiency of one-way permutation-based hash functions. In Foundations of Computer Science, 1999. 40th Annual Symposium on, pages $535-542,1999$.

[16] M. Mahmoody, H. K. Maji, and M. Prabhakaran. Limits of random oracles in secure computation. Technical Report 1205.3554v1, arXiv, 2012. arXiv:1205.3554v1.

[17] A. McGregor, I. Mironov, T. Pitassi, O. Reingold, K. Talwar, and S. P. Vadhan. The limits of two-party differential privacy. Electronic Colloquium on Computational Complexity (ECCC), page 106, 2011. Preliminary version in FOCS'10.

[18] R. C. Merkle. Secure communications over insecure channels. In SIMMONS: Secure Communications and Asymmetric Cryptosystems, 1982.

[19] I. Mironov, O. Pandey, O. Reingold, and S. P. Vadhan. Computational differential privacy. In Advances in Cryptology - CRYPTO '09, pages 126-142, 2009. 
[20] D. Pointcheval and J. Stern. Security proofs for signature schemes. In Advances in Cryptology - EUROCRYPT'96, pages 387-398, 1996.

[21] O. Reingold, L. Trevisan, and S. P. Vadhan. Notions of reducibility between cryptographic primitives. In Theory of Cryptography, First Theory of Cryptography Conference, TCC 2004, volume 2951 of Lecture Notes in Computer Science, pages 1-20. Springer, 2004.

[22] S. Rudich. The use of interaction in public cryptosystems. In Proceedings of the 11th Annual International Cryptology Conference on Advances in Cryptology, CRYPTO '91, pages 242-251, 1992.

[23] M. Santha and U. V. Vazirani. Generating quasi-random sequences from semi-random sources. J. Comput. Syst. Sci., 33(1):75-87, 1986.

[24] D. Simon. Finding collisions on a one-way street: Can secure hash functions be based on general assumptions? In Advances in Cryptology - EUROCRYPT '98, pages 334-345, 1998.

[25] H. Wee. One-way permutations, interactive hashing and statistically hiding commitments. In Theory of Cryptography, First Theory of Cryptography Conference, TCC 2004, pages 419-433, 2007.

\section{A Proving Lemma 15}

We start by proving Lemma 15 for normal-form protocols, defined below. In Appendix A.1 we extend the proof to arbitrary protocols. In the following, for a view $v$ describing an execution of an oracle-aided protocol $\pi$, we let $\ell_{i}(v)$ be the number of queries made (by the non-idle party) in round $i$ according to $v$.

Definition 61 (normal-form protocols). An oracle-aided protocol $\pi$ is in normal-form if $\ell_{i}(v) \leq 1$ for every possible view $v$ and every round $i$, that is, if a party makes at most a single query to the oracle in each communication round.

Fix a normal-form, oracle-aided protocol $\pi$, a simple function family $\mathcal{F}$, and $0<\delta \leq 1 / \ell$. Define the following oracle-aided algorithm.

\section{Algorithm 62 (Finder).}

Input: a transcript $\bar{t}$ and a list $\mathcal{I}$ of query/answer pairs.

Oracle: $f \in \mathcal{F}$.

Operation: While there exists a query $q$ with $(q, \cdot) \notin \mathcal{I}$ and $p_{q}>\delta / 32$, where $p_{q}$ is the probability that $q$ was asked either by $\mathrm{A}$ or $\mathrm{B}$ in a random sample from $\mathcal{V} \mathcal{I} \mathcal{E} \mathcal{W}_{\text {Ind }}^{\mathcal{F}, \pi}(\bar{t}, \mathcal{I}):^{22}$

Add $(q, f(q))$ to $\mathcal{I}$ (choose the lexicographically first $q$ if there are more than one).

\footnotetext{
${ }^{22}$ Recall that the distribution $\mathcal{V I E} \mathcal{W}_{\text {Ind }}^{\mathcal{F}, \pi}(\bar{t}, \mathcal{I})$ stands for a random, independent joint view of $\pi$, which is consistent with the transcript $\bar{t}$ and the query/answer list $\mathcal{I}$.
} 
Namely, algorithm Finder considers executions of $\pi$ that are consistent with the given partial transcript and a set of "known" query/answer pairs, in which the views of the parties are independent (since $\mathcal{F}$ is a simple family, it means that the parties share no intersecting query - a common query whose answer in not described in the given information). The goal of Finder is to find all "heavy queries": those queries that have substantial probability of being asked by one of the parties in a random such execution.

We prove Lemma 15 (for the case of normal-form protocols) by showing that Finder is a $\left(64 / \delta^{2}, \ell \delta\right)$-DependencyFinder for $(\mathcal{F}, \pi)$. The heart of the proof is in the following lemma, yielding that in a random execution of $\mathrm{CF}=\mathrm{CF}(\mathcal{F}, \pi$, Finder $)$, the probability that the views of the parties "become dependent", in any given round, is small.

Lemma 63. Let $\bar{t}$ be a (possibly partial) transcript of (the normal-form protocol) $\pi$, let $\mathcal{I}$ be a set of query/answer pairs, let $\operatorname{Ind}=\operatorname{Ind}^{\pi, \mathcal{F}}(\bar{t}, \mathcal{I})$, let $\mathcal{D}=\mathcal{V} \mathcal{I E} \mathcal{W}_{\text {Ind }}(\bar{t}, \mathcal{I})$, and let $\delta>0$ be such that $\operatorname{Pr}_{v \leftarrow \mathcal{D}}[q \in v \wedge(q, \cdot) \notin \mathcal{I}] \leq \delta \leq \frac{1}{4 \ell}$ for every query $q$. Then, the following hold:

1. There exists a product distribution $\mathcal{C}$ over $\operatorname{Supp}\left(\mathcal{D}_{\mathrm{A}}\right) \times \operatorname{Supp}\left(\mathcal{D}_{\mathrm{B}}\right)$ with $\operatorname{SD}(\mathcal{D}, \mathcal{C}) \leq 2 \ell \delta$.

2. Let $\mathcal{D}^{+}$be the value of $\operatorname{view}(\omega)_{|\bar{t}|+1}$ for $\omega \leftarrow \Omega_{\mathrm{Ind}}(\bar{t}, \mathcal{I})$, then $\operatorname{Pr}_{v \leftarrow \mathcal{D}^{+}}\left[\operatorname{Dependent}_{\mathcal{I}}(v)\right] \leq$ $4 \delta \cdot \operatorname{Pr}_{v \leftarrow \mathcal{D}^{+}}\left[\ell_{|\bar{t}|+1}(v)=1\right]$.

We prove Lemma 63 in Appendix A.2, but first use it for proving Lemma 15. We start by showing that Algorithm Finder (see Algorithm 62) is a good DependencyFinder for $(\mathcal{F}, \pi)$, proving Lemma 15 for normal-form protocols.

Claim 64. Algorithm Finder is a $\left(64 / \delta^{2}, \ell \delta\right)$-DependencyFinder for $(\mathcal{F}, \pi)$.

Proof. The following random variables are defined with respect to a random execution of $\mathrm{CF}=$ $\operatorname{CF}(\mathcal{F}, \pi$, Finder $)$. Let $V=\operatorname{view}(\omega)_{j}$, i.e., the $j$ long prefix of the full view implied by the triplet $\omega \in \Omega$ chosen in the first step of CF. We let $\bar{T}$ denote the (j-round) transcript in $V$. For $i \in[j]$ let $I_{i}$ denote the value of $\mathcal{I}_{i}$ computed in CF, and for $2 \leq i \leq j$ let FirstDep $_{i}=\operatorname{Dependent}_{I_{i-1}}\left(V_{i}\right) \wedge$ $\neg \operatorname{Dependent}_{I_{i-2}}\left(V_{i-1}\right)$ (where $I_{0}=\emptyset$ ). We start by bounding the probability of Dependent $I_{j}(V)$, which yields the first property required for being a $(\cdot, \ell \delta)$-DependencyFinder for $(\mathcal{F}, \pi)$, and then complete the proof by bounding the number of queries made in CF.

Bounding the probability of dependencies. The following claim bounds the probability that a single "round" of CF causes dependencies.

Claim 65. $\operatorname{Pr}\left[\right.$ FirstDep $\left._{i}\right] \leq \frac{\delta}{8} \cdot \mathrm{E}\left[\ell_{i}(V)\right]$ for every $2 \leq i \leq j$.

Proof. In the following we fix $2 \leq i \leq j$ and a value for $\left(\bar{T}_{1, \ldots, i-1}, I_{i-1}\right)$, and prove (the slightly stronger fact) that the claim holds even under any such fixing. We next bound the probability that (under this fixing) the $i$ 'th round of $\pi$ causes a collision. Recall that by Process CF, we obtained $I_{i-1}=I_{i-2} \cup$ Finder $^{f}\left(\bar{T}_{1, \ldots, i-1}, I_{i-2}\right)$. The definition of Finder yields that

$$
\operatorname{Pr}\left[q \in V_{i-1} \wedge(q, \cdot) \notin I_{i-1} \mid \neg \text { Dependent }_{I_{i-1}}\left(V_{i-1}\right)\right] \leq \delta / 32
$$


for any query $q$. We can hence apply Lemma 63(2) with respect to the function family $\mathcal{F}$, protocol $\pi$, transcript $\bar{T}_{1, \ldots, i-1}$ and query/answer list $I_{i-1}$, yielding that

$$
\operatorname{Pr}\left[\operatorname{Dependent}_{I_{i-1}}\left(V_{i}\right) \mid \neg \operatorname{Dependent}_{I_{i-1}}\left(V_{i-1}\right)\right] \leq \frac{\delta}{8} \cdot \operatorname{Pr}\left[\ell_{i}(V)=1 \mid \neg \text { Dependent }_{I_{i-1}}\left(V_{i-1}\right)\right]
$$

We conclude that

$$
\begin{aligned}
& \operatorname{Pr}\left[\text { FirstDep }_{i}\right] \\
& =\operatorname{Pr}\left[\text { Dependent }_{I_{i-1}}\left(V_{i}\right) \wedge \neg \text { Dependent }_{I_{i-2}}\left(V_{i-1}\right)\right] \\
& \leq \operatorname{Pr}\left[\text { Dependent }_{I_{i-1}}\left(V_{i}\right) \wedge \neg \operatorname{Dependent}_{I_{i-1}}\left(V_{i-1}\right)\right] \\
& =\operatorname{Pr}\left[\neg \operatorname{Dependent}_{I_{i-1}}\left(V_{i-1}\right)\right] \cdot \operatorname{Pr}\left[\operatorname{Dependent}_{I_{i-1}}\left(V_{i}\right) \mid \neg \operatorname{Dependent}_{I_{i-1}}\left(V_{i-1}\right)\right] \\
& \leq \operatorname{Pr}\left[\neg \operatorname{Dependent}_{I_{i-1}}\left(V_{i-1}\right)\right] \cdot \frac{\delta}{8} \cdot \operatorname{Pr}\left[\ell_{i}(V)=1 \mid \neg \operatorname{Dependent}_{I_{i-1}}\left(V_{i-1}\right)\right] \\
& \leq \frac{\delta}{8} \cdot \mathrm{E}\left[\ell_{i}(V)\right]
\end{aligned}
$$

The first inequality holds since $\mathcal{F}$ is a simple family (hence, $\operatorname{Dependent}_{\mathcal{I}}(v)=0$ implies $\operatorname{Dependent}_{\mathcal{I}^{\prime}}(v)=0$, for every view $v$ and lists $\left.\mathcal{I}^{\prime} \supseteq \mathcal{I}\right)$ and the second one follows from Equation (30). Finally, since Equation (31) holds conditioned on any fixing of $\left(\bar{T}_{1, \ldots, i-1}, I_{i-1}\right)$, it also holds without this conditioning and the claim follows.

Continuing the proof, Claim 65 yields that

$$
\begin{aligned}
\operatorname{Pr}\left[\text { Dependent }_{I_{j}}(V)\right] & \leq \operatorname{Pr}\left[\bigvee_{2 \leq i \leq j} \text { FirstDep }_{i}\right] \leq \sum_{2 \leq i \leq j} \frac{\delta}{8} \cdot \mathrm{E}\left[\ell_{i}(V)\right] \\
& =\frac{\delta}{8} \mathrm{E}\left[\sum_{2 \leq i \leq j} \ell_{i}(V)\right] \leq \frac{\ell \delta}{8} .
\end{aligned}
$$

The first inequality holds since $\mathcal{F}$ is a simple family and since a first round view is never dependent (the inactive party made no queries). The third inequality holds since $\ell$ bounds the overall number of queries made in any execution of $\pi$. Since for any $d=(\bar{t}, \mathcal{I}) \in \operatorname{Supp}\left(\bar{T}, I_{j}\right)$ it holds that $\operatorname{SD}\left(\mathcal{V I E} \mathcal{W}(d), \mathcal{V I E} \mathcal{W}_{\text {Ind }}(d)\right)=\operatorname{Pr}_{\omega \leftarrow \Omega(d)}[w \notin \operatorname{Ind}(d)]=\operatorname{Pr}_{v \leftarrow \mathcal{V I E} \mathcal{W}(d)}\left[\operatorname{Dependent}_{\mathcal{I}}(v)\right]$, it follows that

$$
\begin{aligned}
\underset{d \leftarrow\left(\bar{T}, I_{j}\right)}{\mathrm{E}}\left[\operatorname{SD}\left(\mathcal{V I E \mathcal { W }}(d), \mathcal{V} \mathcal{I} \mathcal{E} \mathcal{W}_{\text {Ind }}(d)\right)\right] & =\underset{d \leftarrow\left(\bar{T}, I_{j}\right)}{\mathrm{E}}\left[\underset{v \leftarrow \mathcal{V} \mathcal{I} \mathcal{E} \mathcal{W}(d)}{\operatorname{Pr}}\left[\operatorname{Dependent}_{\mathcal{I}}(v)\right]\right] \\
& =\operatorname{Pr}\left[\operatorname{Dependent~}_{I_{j}}(V)\right] \\
& \leq \frac{\ell \delta}{8}
\end{aligned}
$$

where the inequality hold by Equation (32). We complete the proof of this part by showing that $\mathcal{V} \mathcal{I} \mathcal{W}_{\text {Ind }}(d)$ is close to some product distribution over the parties' views. The definition of Finder yields that

$$
\operatorname{Pr}\left[q \in V \wedge(q, \cdot) \notin I_{j} \mid \neg \operatorname{Dependent}_{I_{j}}(V),\left(\bar{T}, I_{j}\right)=d\right] \leq \frac{\ell \delta}{32}
$$


for any possible query $q$ and $d \in \operatorname{Supp}\left(\bar{T}, I_{j}\right)$. Therefore, Lemma $63(1)$ yields that

$$
\mathrm{SD}\left(\mathcal{V} \mathcal{I} \mathcal{E} \mathcal{W}_{\text {Ind }}(d), \mathcal{C}(d)\right) \leq \frac{\ell \delta}{16}
$$

for any $d \in \operatorname{Supp}\left(\bar{T}, I_{j}\right)$, where $\mathcal{C}(d)$ is a product distribution over the parties' views. It follows (using the triangle inequality) that $\operatorname{SD}\left(\mathcal{V} \mathcal{I} \mathcal{E} \mathcal{W}_{\text {Ind }}(d),\left(\mathcal{V} \mathcal{I E} \mathcal{W}_{\text {Ind }}(d)_{\mathrm{A}}, \mathcal{V} \mathcal{I E} \mathcal{W}_{\text {Ind }}(d)_{\mathrm{B}}\right)\right) \leq \frac{3}{16} \cdot \ell \delta$ for any $d \in \operatorname{Supp}\left(\bar{T}, I_{j}\right)$, and therefore

$$
\underset{d \leftarrow\left(\bar{T}, I_{j}\right)}{\mathrm{E}}\left[\mathrm{SD}\left(\mathcal{V} \mathcal{I} \mathcal{E} \mathcal{W}_{\text {Ind }}(d),\left(\mathcal{V} \mathcal{I E} \mathcal{W}_{\text {Ind })}(d)_{\mathrm{A}}, \mathcal{V} \mathcal{I} \mathcal{E} \mathcal{W}_{\text {Ind })}(d)_{\mathrm{B}}\right)\right)\right] \leq \frac{3}{16} \cdot \ell \delta
$$

Combining Equations (33) and (36) and the triangle inequality, yields that

$$
\underset{d \leftarrow\left(\bar{T}, I_{j}\right)}{\mathrm{E}}\left[\mathrm{SD}\left(\mathcal{V I E \mathcal { E }}(d),\left(\mathcal{V} \mathcal{I E \mathcal { W }}(d)_{\mathrm{A}}, \mathcal{V} \mathcal{I} \mathcal{E} \mathcal{W}(d)_{\mathrm{B}}\right)\right)\right] \leq \frac{9}{16} \cdot \ell \delta \leq \ell \delta
$$

Bounding the query complexity. We complete the proof by bounding the probability that CF makes too many oracle queries. For $i \in[j]$ let $Q_{i}=\left\{q:(q, \cdot) \in I_{i} \backslash I_{i-1}\right\}$ and let $Q=\bigcup_{i \in[j]} Q_{i}$ (i.e., $Q$ is the set of queries appearing in a query/answer pair of $I_{j}$ ). The heart of the proof is in the following claim.

Claim 66. For every query $q$ it holds that

$$
\sum_{i \in[j]} \operatorname{Pr}\left[q \in Q_{i} \wedge \neg \text { Dependent }_{I_{i-1}}\left(V_{i}\right)\right] \leq \frac{32}{\delta} \cdot \operatorname{Pr}[q \in V] .
$$

Namely, Claim 66 relates the probability that a query is asked by Finder, to the probability that this query is asked by one of the parties in $V$.

Proof. Fix $i \in[j]$ for a moment, and assume that during the $i^{\prime}$ th call to Finder on input $\left(\bar{t}^{*}, \cdot\right)$, algorithm Finder is about to ask a query $q$ and let $\mathcal{I}^{*}$ be the value of $\mathcal{I}$ at this moment. The definition of Finder tells us that

$$
\operatorname{Pr}\left[q \in V_{i} \mid \mathcal{I}^{*}, \bar{t}^{*}, \neg \operatorname{Dependent}_{\mathcal{I}^{*}}\left(V_{i}\right)\right] \geq \delta / 32,
$$

where the conditioning on $\left(\mathcal{I}^{*}, \bar{t}^{*}\right)$ means that $V_{i}$ is consistent with $\mathcal{I}^{*}$ and $\bar{t}^{*}$. Applying a simple Bayes' rule, it follows that

$$
\begin{aligned}
\operatorname{Pr}\left[q \in V_{i} \mid \mathcal{I}^{*}, \bar{t}^{*}\right] & \geq \operatorname{Pr}\left[q \in V_{i} \wedge \neg \operatorname{Dependent}_{\mathcal{I}^{*}}\left(V_{i}\right) \mid \mathcal{I}^{*}, \bar{t}^{*}\right] \\
& =\operatorname{Pr}\left[\neg \text { Dependent }_{\mathcal{I}^{*}}\left(V_{i}\right) \mid \mathcal{I}^{*}, \bar{t}^{*}\right] \cdot \operatorname{Pr}\left[q \in V_{i} \mid \mathcal{I}^{*}, \bar{t}^{*}, \neg \text { Dependent }_{\mathcal{I}^{*}}\left(V_{i}\right)\right] \\
& \geq \operatorname{Pr}\left[\neg \operatorname{Dependent}_{\mathcal{I}^{*}}\left(V_{i}\right) \mid \mathcal{I}^{*}, \bar{t}^{*}\right] \cdot \frac{\delta}{32} .
\end{aligned}
$$

For $i \in[j]$ let $\mathcal{S}_{i}(q)$ be the set of $\left(\mathcal{I}^{*}, \bar{t}^{*}\right)$ pairs that cause Finder to ask the query $q$ in the $i$ 'th round of CF - assuming that $w \in \Omega$ (chosen in CF) is consistent with $\left(\mathcal{I}^{*}, \bar{t}^{*}\right)$, then Finder asks the 
query $q$ in the $i$ 'th round of $\mathrm{CF}$, and the value of $\mathcal{I}$ before it does so is $\mathcal{I}^{*}$. It follows that

$$
\begin{aligned}
\operatorname{Pr}[q \in V] & \geq \sum_{i \in[j]} \sum_{\left(\mathcal{I}^{*}, \bar{t}^{*}\right) \in \mathcal{S}_{i}(q)} \operatorname{Pr}\left[w \text { is consistent with }\left(\mathcal{I}^{*}, \bar{t}^{*}\right)\right] \cdot \operatorname{Pr}\left[q \in V \mid \mathcal{I}^{*}, \bar{t}^{*}\right] \\
& \geq \sum_{i \in[j]} \sum_{\left(\mathcal{I}^{*}, \bar{t}^{*}\right) \in \mathcal{S}_{i}(q)} \operatorname{Pr}\left[w \text { is consistent with }\left(\mathcal{I}^{*}, \bar{t}^{*}\right)\right] \cdot \frac{\delta}{32} \cdot \operatorname{Pr}\left[\neg \text { Dependent }_{\mathcal{I}^{*}}\left(V_{i}\right) \mid \mathcal{I}^{*}, \bar{t}^{*}\right] \\
& \geq \frac{\delta}{32} \cdot \sum_{i \in[j]} \operatorname{Pr}\left[q \in Q_{i} \wedge \neg \text { Dependent }_{I_{i-1}}\left(V_{i}\right)\right] .
\end{aligned}
$$

The first inequality holds since a query is asked at most once in CF (and hence we are summing over disjoint events), the second one by Equation (38) and the last one holds since $\mathcal{F}$ is a simple family.

Let $\widetilde{\mathrm{CF}}$ be the variant of CF that aborts in case Dependent ${ }_{I_{i-1}}\left(V_{i}\right)=1$ for some $i \in[j]$ (i.e., $\widetilde{\mathrm{CF}}$ aborts right after computing $\left.I_{i-1}\right)$. Let $\widetilde{Q}_{i}$ be the respective analogs of $Q_{i}$ defined with respect to a random execution of $\widetilde{C F}$, and let $\widetilde{Q}=\bigcup_{i \in[j]} \widetilde{Q}_{i}$ (i.e., $\widetilde{Q}$ denote all queries asked by Finder in $\widetilde{C F}$ ). The same calculation done in Equation (32) yields that

$$
S D(Q, \widetilde{Q}) \leq \ell \delta / 8
$$

In the following we bound the number of queries made by Finder in $\widetilde{C F}$, and derive a similar bound on CF.

A simple argument yields that $\operatorname{Pr}\left[q \in \widetilde{Q}_{i}\right] \leq \operatorname{Pr}\left[q \in Q_{i} \wedge \neg\right.$ Dependent $\left._{I_{i-1}}\left(V_{i}\right)\right]$, for every $i \in[j]$ and every query $q$. Thus, Claim 66

$$
\sum_{i \in[j]} \operatorname{Pr}\left[q \in Q_{i} \wedge \neg \text { Dependent }_{I_{i-1}}\left(V_{i}\right)\right] \leq \frac{32}{\delta} \cdot \operatorname{Pr}[q \in V]
$$

yields that

$$
\operatorname{Pr}[q \in \widetilde{Q}]=\sum_{i \in[j]} \operatorname{Pr}\left[q \in \widetilde{Q}_{i}\right] \leq \frac{32}{\delta} \cdot \operatorname{Pr}[q \in V]
$$

for every query $q$. It follows that

$$
\mathrm{E}[|\widetilde{Q}|]=\mathrm{E}\left[\sum_{q} \chi_{\widetilde{Q}}(q)\right] \leq \frac{32}{\delta} \cdot \mathrm{E}\left[\sum_{q} \chi_{V}(q)\right] \leq 32 \ell / \delta .
$$

The first inequality holds by Equation (40) and linearity of expectation and the last one since at most $\ell$ queries are asked in $V$ (where $\chi_{x}(q)=1$ if $q \in x$ and $\chi_{x}(q)=0$ otherwise).

A Markov argument yields that $\operatorname{Pr}\left[|\widetilde{Q}|>64 / \delta^{2}\right] \leq \ell \delta / 2$. Hence, Equation (39) yields that $\operatorname{Pr}\left[|Q|>64 / \delta^{2}\right] \leq \ell \delta / 2+\ell \delta / 8<\ell \delta$. 


\section{A.1 Handling Non Normal-Form Protocols}

In this section we show how to construct a DependencyFinder for every simple function family and every oracle-aided protocol (possibly not in normal form). We do this by showing how to use a DependencyFinder for the normal form variant of a protocol, defined below, to construct a DependencyFinder (of the same quality) for the original protocol.

Definition 67 (the normal-form variant of a protocol). Given an $\ell$-query oracle-aided protocol $\pi$, we define its normal form variant $\pi_{N}$ as follows: the parties of $\pi_{N}$ act as in $\pi$ while sending additional "dummy" messages; following each oracle query made through the execution, the parties interact in a "dummy round" - the active party sends $\perp$ to the other party who answers with $\perp$. In addition, before sending the next message of $\pi$, the parties interact in $(\ell-k)$ consecutive dummy rounds, where $k$ is the number of oracle queries made by the active party in the current round. ${ }^{23}$

Lemma 68. Let $\mathcal{F}$ be a function family, let $\pi$ be an oracle-aided protocol and let $\pi_{N}$ be its normal form variant. Assume $\left(\mathcal{F}, \pi_{N}\right)$ has a $(T, \varepsilon)$-DependencyFinder, then $(\mathcal{F}, \pi)$ has a $(T, \varepsilon)$ DependencyFinder.

The straightforward proof of Lemma 68 is given below, but first let us use it for concluding the proof of Lemma 15.

Proof of Lemma 15. Let $\mathcal{F}$ be a simple function family, let $\pi=(\mathrm{A}, \mathrm{B})$ be an $\ell$-query oracle-aided protocol and let $\pi_{\mathrm{N}}$ be its normal-form variant. Since $\pi_{\mathrm{N}}$ is in normal form according to Definition 61, Claim 64 yields that $\left(\mathcal{F}, \pi_{\mathrm{N}}\right)$ has a $\left(64 / \delta^{2}, \ell \delta\right)$-DependencyFinder for any $\delta \leq 1 / \ell$. Hence, Lemma 68 yields that the same holds for $(\mathcal{F}, \pi)$.

Proof of Lemma 68. Let $\mathcal{F}, \pi$ and $\pi_{\mathrm{N}}$ be as in the statement of the lemma, and let Finder $\mathrm{N}_{\mathrm{N}}$ be a $(T, \varepsilon)$-DependencyFinder for $\left(\mathcal{F}, \pi_{\mathrm{N}}\right)$. We define the DependencyFinder for $(\mathcal{F}, \pi)$ as follows:

Algorithm 69 (Finder).

Input: a transcript $\bar{t}$ of $\pi$ and a list $\mathcal{I}$ of query/answer pairs.

Oracle: $f \in \mathcal{F}$.

Operation:

1. Create the transcript $\bar{t}_{N}$ from $\bar{t}$ by inserting $2 \ell$ strings ' $\perp$, ,', following each but the last message in $\bar{t}$.

2. For $k=1$ to $\ell$ do:

(a) $\operatorname{Set} \mathcal{I}=\mathcal{I} \cup \operatorname{Finder}_{N}\left(\mathcal{I}, \bar{t}_{N}\right)$.

(b) $\operatorname{Set} \bar{t}_{N}=\bar{t}_{N}, \perp, \perp$

It is easy to verify that a random output of $\mathrm{CF}_{\mathrm{N}}$ can be sampled by applying a (deterministic) injective function $\mathrm{M}$ to a random output of $\mathrm{CF}$, where $\mathrm{M}$ preserves the number of queries in the input (specifically, $\mathrm{M}$ is simply the padding function described in the first line of Algorithm 69). This observation immediately yields the required bound on the number of oracle queries done in

\footnotetext{
${ }^{23}$ Note that each round in the original protocol is replaced by $\ell$ rounds in its normal-form variant, hence, concealing the number of actual oracle-queries made in each round.
} 
$\mathrm{CF}$, since these outputs determine the number of queries made to the oracle. To prove that the first property required by Definition 14 also holds (see the equation below), we also note that a random sample of $\mathcal{V} \mathcal{I E} \mathcal{W}^{\mathcal{F}, \pi_{\mathrm{N}}}\left(d_{\mathrm{N}}\right)$, for $d_{\mathrm{N}} \in \operatorname{Supp}\left(\mathrm{CF}_{\mathrm{N}}\right)$, can be sampled by applying a (deterministic) injective function to $\mathcal{V} \mathcal{I} \mathcal{E} \mathcal{W}^{\mathcal{F}, \pi}\left(\mathrm{M}^{-1}\left(d_{\mathrm{N}}\right)\right)$. It follows that

$$
\begin{aligned}
& \underset{d \leftarrow \mathrm{CF}}{\mathrm{E}}\left[\mathrm{SD}\left(\mathcal{V I E} \mathcal{W}^{\mathcal{F}, \pi}(d),\left(\mathcal{V I E} \mathcal{W}^{\mathcal{F}, \pi}(d)_{\mathrm{A}}, \mathcal{V} \mathcal{I E} \mathcal{W}^{\mathcal{F}, \pi}(d)_{\mathrm{B}}\right)\right)\right] \\
& =\underset{d \leftarrow \mathrm{CF}}{\mathrm{E}}\left[\mathrm{SD}\left(\mathcal{V} \mathcal{I} \mathcal{E} \mathcal{W}^{\mathcal{F}, \pi_{\mathrm{N}}}(d),\left(\mathcal{V} \mathcal{I} \mathcal{W} \mathcal{W}^{\mathcal{F}, \pi_{\mathrm{N}}}(d)_{\mathrm{A}}, \mathcal{V} \mathcal{I} \mathcal{E} \mathcal{W}^{\mathcal{F}, \pi_{\mathrm{N}}}(d)_{\mathrm{B}}\right)\right)\right] \\
& \leq \varepsilon
\end{aligned}
$$

concluding the proof of the lemma.

\section{A.2 Proving Lemma 63}

The following discussion is with respect to fixed values of $\bar{t}$ and $\mathcal{I}$, where $\operatorname{Ind}, \mathcal{D}$ and $\mathcal{D}^{+}$are defined with respect to these values as in the statement of Lemma 63.

Towards proving Lemma 63, we make the following observations: in Claim 70 we show that $\mathcal{D}$ is distributed as some product distribution, under the independence condition. In Claim 72 , we use the first observation to express $\mathcal{D}$ as a uniform sampled edge of a dense bipartite graph. ${ }^{24}$

\section{A.2.1 Product Characterization}

Claim 70. There exist two distributions $\mathcal{A}$ and $\mathcal{B}$ with $\mathcal{D}=(\mathcal{A} \times \mathcal{B}) \mid$ Ind.

Proof. We show that we can write $\mathcal{D}(v)=\gamma_{\mathrm{A}}\left(v_{\mathrm{A}}\right) \cdot \gamma_{\mathrm{B}}\left(v_{\mathrm{B}}\right) \cdot c$ for every $v=\left(v_{\mathrm{A}}, v_{\mathrm{B}}\right) \in \operatorname{Supp}(\mathcal{D})$, where $\gamma_{A}$ and $\gamma_{B}$ are appropriate functions, and $c$ is a global constant. This would imply the claim, letting $\mathcal{A}$ be the distribution over $\operatorname{Supp}\left(\mathcal{D}_{\mathrm{A}}\right)$ with $\mathcal{A}\left(v_{\mathrm{A}}\right)=c_{\mathcal{A}} \cdot \gamma_{\mathrm{A}}\left(v_{\mathrm{A}}\right)$, and $\mathcal{B}$ be the distribution over $\operatorname{Supp}\left(\mathcal{D}_{\mathrm{B}}\right)$ with $\mathcal{B}\left(v_{\mathrm{B}}\right)=c_{\mathcal{B}} \cdot \gamma_{\mathrm{B}}\left(v_{\mathrm{B}}\right)$, for the appropriate constants $c_{\mathcal{A}}$ and $c_{\mathcal{B}}$.

Proposition 8 yields that

$$
\mathcal{D}(v)=\frac{\operatorname{Pr}_{\Omega}\left[r_{\mathrm{A}}, r_{\mathrm{B}}\right] \cdot \alpha_{v_{\mathrm{A}} \mid v_{\mathrm{B}}}^{\mathcal{I}} \cdot \alpha_{v_{\mathrm{B}} \mid v_{\mathrm{A}}}^{\mathcal{I}}}{\operatorname{Pr}_{\Omega \mid \mathcal{I}}[\bar{t}, \text { Ind }]}
$$

for every $v=\left(r_{\mathrm{A}}, r_{\mathrm{B}}, \cdot\right) \in \operatorname{Supp}(\mathcal{D})$. Since the random coins of the parties are chosen independently, it holds that $\operatorname{Pr}_{\Omega}\left[r_{\mathrm{A}}, r_{\mathrm{B}}\right]=\operatorname{Pr}_{\Omega}\left[r_{\mathrm{A}}\right] \cdot \operatorname{Pr}_{\Omega}\left[r_{\mathrm{B}}\right]$. In addition, the definition of $\mathcal{D}$ yields that $\alpha_{v_{\mathrm{A}} \mid v_{\mathrm{B}}}^{\mathcal{I}} \alpha_{v_{\mathrm{A}}}^{\mathcal{I}}$ and that $\alpha_{v_{\mathrm{B}} \mid v_{\mathrm{A}}}^{\mathcal{I}}=\alpha_{v_{\mathrm{B}}}^{\mathcal{I}}$, for every $v \in \operatorname{Supp}(\mathcal{D})$. Taking $\gamma_{\mathrm{A}}\left(v_{\mathrm{A}}\right):=\operatorname{Pr}_{\Omega}\left[r_{\mathrm{A}}\right] \cdot \alpha_{v_{\mathrm{A}}}^{\mathcal{I}}$ and $\gamma_{\mathrm{B}}\left(v_{\mathrm{B}}\right):=\operatorname{Pr}_{\Omega}\left[r_{\mathrm{B}}\right] \cdot \alpha_{v_{\mathrm{B}}}^{\mathcal{I}}$, we obtain the desired result.

\section{A.2.2 Graph Characterization}

We use the above product characterization to show that we can think of $\mathcal{D}$ as a distribution over random edges of some bipartite graph $G$. We show that $\mathcal{D}$ is close to being a product distribution by showing that the graph is dense. Specifically, we show that every vertex in $G$ is connected to most of the vertices on the other side.

\footnotetext{
${ }^{24}$ Observations of similar spirit were done in [1]. Much the following text is taken verbatim from [1].
} 
Definition 71 (The graph $G$ ). Define the bipartite graph $G=\left(V_{\mathcal{A}}, V_{\mathcal{B}} ; E\right)$ as follows: let $\mathcal{A}$ and $\mathcal{B}$ be as the distribution guaranteed by Claim 70. Every node $a \in V_{\mathcal{A}}$ has a corresponding view view $(a)$ of $\mathrm{A}$ in the support of $\mathcal{A}$; we let the number of nodes corresponding to a view $v_{\mathrm{A}}$ be proportional to $\mathcal{A}\left(v_{\mathrm{A}}\right)$, meaning that $\mathcal{A}$ corresponds to the uniform distribution over the left-side vertices $V_{\mathcal{A}}$. Similarly, every node $b \in V_{\mathcal{B}}$ has a corresponding view $\operatorname{view}_{\mathrm{B}}(b)$ of $\mathrm{B}$, such that $\mathcal{B}$ corresponds to the uniform distribution over $V_{\mathcal{B}}$. Finally, let $E=\left\{(a, b) \in\left(V_{\mathcal{A}} \times V_{\mathcal{B}}\right) \cap\right.$ Ind $\}$.

Claim 70 yields that $\mathcal{D}$ corresponds to the distribution obtained by letting $(a, b) \leftarrow E$, and choosing $\left(\operatorname{view}_{\mathcal{A}}(a), \operatorname{view}_{\mathcal{B}}(b)\right)$. We next show that this graph must be dense.

Claim 72. Let $G=\left(V_{\mathcal{A}}, V_{\mathcal{B}} ; E\right)$ be as in Definition 71. Then $d(a) \geq\left|V_{\mathcal{B}}\right| \cdot(1-2 \ell \delta)$ for every $a \in V_{\mathcal{A}}$, and $d(b) \geq\left|V_{\mathcal{A}}\right| \cdot(1-2 \ell \delta)$ for every $b \in V_{\mathcal{B}}$, where $d(x)$ stands for the degree of the vertex $x$.

Proof. Since, by assumption, $\operatorname{Pr}_{v \leftarrow \mathcal{D}}[q \in v \wedge(q, \cdot) \notin \mathcal{I}] \leq \delta$ for every query $q$, since $\ell$ bounds the query complexity of $\pi$, and finally since $\mathcal{F}$ is simple, we have that

$$
\underset{v_{\mathrm{A}} \leftarrow \mathcal{D}_{\mathrm{A}}}{\operatorname{Pr}}\left[\text { Dependent }_{\mathcal{I}}\left(v_{\mathrm{A}}, v_{\mathrm{B}}\right)\right] \leq \ell \delta
$$

for every fixed $v_{\mathrm{B}} \in \operatorname{Supp}\left(\mathcal{D}_{\mathrm{B}}\right)$, and the analogous condition for every fixed $v_{\mathrm{A}} \in \operatorname{Supp}\left(\mathcal{D}_{\mathrm{A}}\right)$. For a vertex $a \in V_{\mathcal{A}}$, let $\stackrel{\nsim}{E}(a)=\left\{b \in V_{\mathcal{B}}:(a, b) \notin E\right\}$. We next show that $\sum_{b \in E(a)} d(b) \leq \ell \delta \cdot|E|$ for every $a \in V_{\mathcal{A}}$. Note that the probability of a vertex $x$ being chosen when selecting a random edge in $E$ is $\frac{d(x)}{|E|}$. Assuming that $\sum_{b \in \mathscr{E}(a)} \frac{d(b)}{|E|}>\ell \delta$, then $\operatorname{Pr}_{v_{\mathrm{B}} \leftarrow \mathcal{D}_{\mathrm{B}}}\left[\operatorname{Dependent}_{\mathcal{I}}\left(\operatorname{view}_{\mathrm{A}}(a), v_{\mathrm{B}}\right)\right]>\ell \delta$, contradicting Equation (43). An analogous argument shows that $\sum_{a \in \mathscr{E}(b)} d(a) \leq \ell \delta \cdot|E|$ for every $b \in V_{\mathcal{B}}$, and we conclude the proof using the following claim:

Fact 73. [1, claim 4.6] Let $G=\left(V_{\mathcal{A}}, V_{\mathcal{B}} ; E\right)$ be a nonempty bipartite graph. Assume there exists $\gamma \leq 1 / 2$ such that $|\stackrel{\sim}{E}(v)| \leq \gamma|E|$ for every vertex $\left.v \in\left(V_{\mathcal{A}} \cup V_{\mathcal{B}}\right)\right)$, then $d(a) \geq\left|V_{\mathcal{B}}\right| \cdot(1-2 \gamma)$ for every $a \in V_{\mathcal{A}}$, and $d(b) \geq\left|V_{\mathcal{A}}\right| \cdot(1-2 \gamma)$ for every $b \in V_{\mathcal{B}}$.

We now use the above claims to prove Lemma 63.

Proof of Lemma 63. The first part of the lemma immediately follows from Claim 72 . We prove the second part of the lemma by showing that

$$
\underset{v \leftarrow \mathcal{D}^{+} \mid v_{\mathrm{B}}=v_{\mathrm{B}}^{+}}{\operatorname{Pr}}\left[\operatorname{Dependent}_{\mathcal{I}}\left(v_{\mathrm{A}}, v_{\mathrm{B}}^{+}\right)\right] \leq 4 \delta \cdot \ell_{|\bar{t}|+1}\left(v_{\mathrm{B}}^{+}\right)
$$

for every $v_{\mathrm{B}}^{+} \in \operatorname{Supp}\left(\mathcal{D}_{\mathrm{B}}^{+}\right)$, where we assume for concreteness that $\mathrm{B}$ is active in the $(|\bar{t}|+1)$ round of $\pi$. Since Equation (44) trivially holds in case $\ell_{|\bar{t}|+1}\left(v_{\mathrm{B}}^{+}\right)=0$, in the following we prove it for $\ell_{|\bar{t}|+1}\left(v_{\mathrm{B}}^{+}\right)=1$ (recall that, by definition, $\ell_{|\bar{t}|+1}\left(v_{\mathrm{B}}^{+}\right) \leq 1$ ).

Fix such view $v_{\mathrm{B}}^{+} \in \operatorname{Supp}\left(\mathcal{D}_{\mathrm{B}}^{+}\right)$, let $v_{\mathrm{B}}^{\prime} \in \operatorname{Supp}\left(\mathcal{D}_{\mathrm{B}}\right)$ be its $|\bar{t}|$-round prefix and let $q^{\prime}$ be the query asked in its $(|\bar{t}|+1)$ round. We assume without loss of generality that $\left(q^{\prime}, \cdot\right) \notin \mathcal{I}$, as otherwise the proof is trivial. Fix further $b \in V_{\mathrm{B}}$ with $\operatorname{view}_{\mathrm{B}}(b)=v_{\mathrm{B}}^{\prime}$, let $N(b)$ be $b$ 's neighbors in $G$ and let $\mathcal{S}=\left\{a \in N(b): q^{\prime} \in \operatorname{view}_{\mathrm{A}}(a)\right\}$. Since $\operatorname{Pr}_{v \leftarrow \mathcal{D}}[q \in v \wedge(q, \cdot) \notin \mathcal{I}] \leq \delta$, we have that

$$
\frac{\sum_{a \in \mathcal{S}} d(a)}{|E|} \leq \delta
$$


and therefore

$$
\operatorname{Pr}_{a \leftarrow N(b)}[a \in \mathcal{S}]=\frac{|\mathcal{S}|}{d(b)} \leq \frac{|\mathcal{S}|}{(1-2 \ell \delta)\left|V_{\mathcal{A}}\right|} \leq \frac{|\mathcal{S}|\left|V_{\mathcal{B}}\right|}{(1-2 \ell \delta)|E|} \leq \frac{\sum_{a \in \mathcal{S}} d(a)}{(1-2 \ell \delta)^{2}|E|} \leq \frac{\delta}{(1-2 \ell \delta)^{2}} \leq 4 \delta
$$

The first and third inequalities hold by Claim 72, the second since $|E| \leq\left|V_{\mathcal{A}}\right|\left|V_{\mathcal{B}}\right|$, and the fourth by Equation (45). Since Equation (46) holds for every $b \in V_{\mathrm{B}}$ with view $\mathrm{B}_{\mathrm{B}}(b)=v_{\mathrm{B}}^{\prime}$, it follows that

$$
\operatorname{Pr}_{v \leftarrow \mathcal{D}^{+} \mid v_{\mathrm{B}}=v_{\mathrm{B}}^{+}}\left[q^{\prime} \in v_{\mathrm{A}}\right] \leq 4 \delta
$$

Finally, since $\mathcal{F}$ is a simple family, Equation (47) yields that $\operatorname{Pr}_{v \leftarrow \mathcal{D}^{+} \mid v_{\mathrm{B}}=v_{\mathrm{B}}^{+}}\left[\right.$Dependent $\left._{\mathcal{I}}\left(v_{\mathrm{A}}, v_{\mathrm{B}}^{+}\right)\right] \leq$ $4 \delta$, concluding the proof of Equation (44), and thus the proof of the lemma. 